\title{
Identification and Functional Characterization of unc5A during Neocortical Regionalization
}

\author{
Dissertation \\ zur Erlangung des Doktorgrades \\ der Mathematisch-Naturwissenschaftlichen Fakultäten \\ der Georg-August-Universität zu Göttingen
}

vorgelegt von

Ahmet Ucar

aus Konya

Göttingen, 2007 
D 7

Referent :

Korreferent :

Tag der mündlichen Prüfung : $\quad 4$ Mai 2007
Professor Dr. P. Gruss

Privatdozent Dr. S. Hoyer-Fender 
Diese Arbeit wurde am

Max-Planck-Institut für biophysikalische Chemie,

Karl-Friedrich-Bonhoefer-Institut

Abteilung: Molekulare Zellbiologie

Direktor: Prof. Dr. Peter Gruss

durchgeführt. 


\section{Table of Contents}

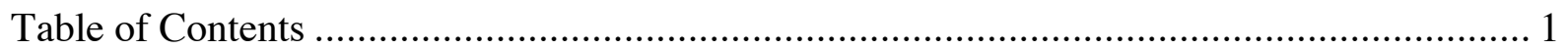

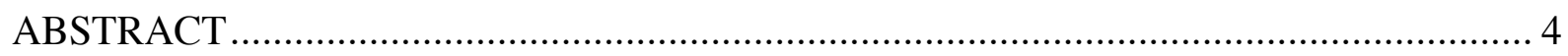

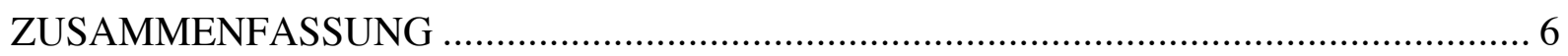

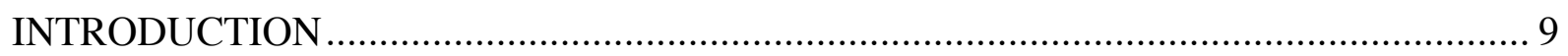

Evolutionary view on brain and cerebral cortex ................................................ 9

Functional and morphological structures of cerebral neocortex .................................. 13

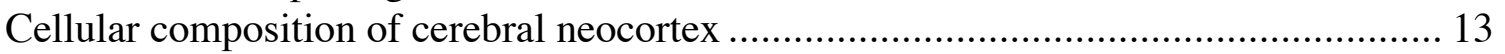

Functional and morphological features of neocortical regions ................................. 15

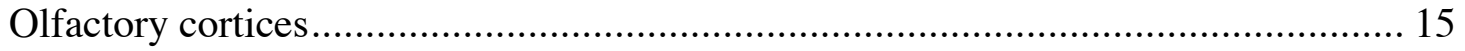

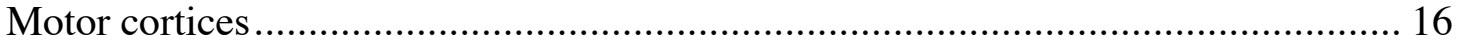

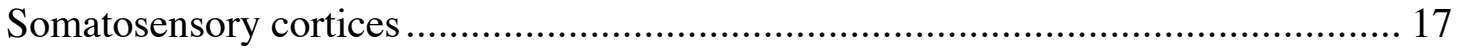

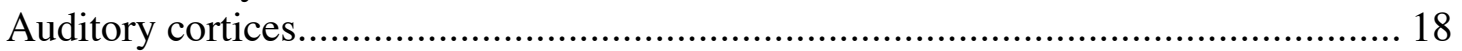

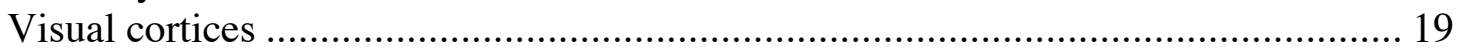

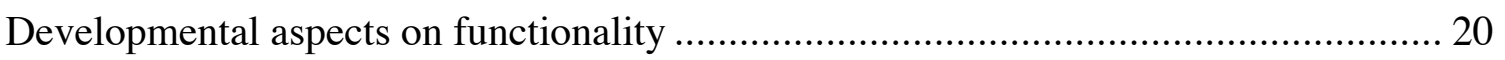

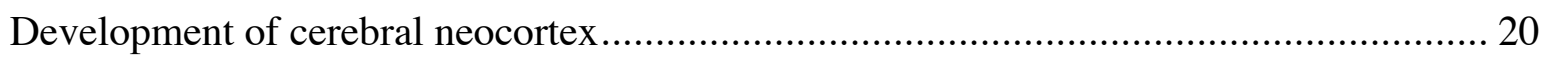

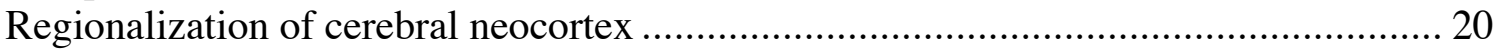

Lamination of cortical plate in cerebral neocortex ................................................... 22

Axon guidance systems in cerebral neocortex development ......................................... 24

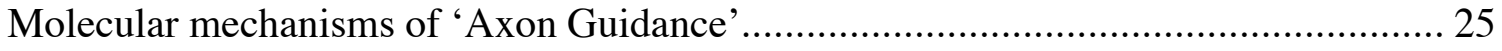

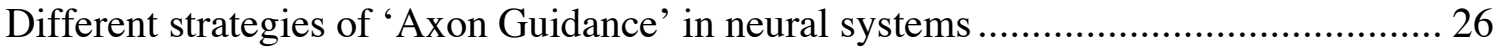

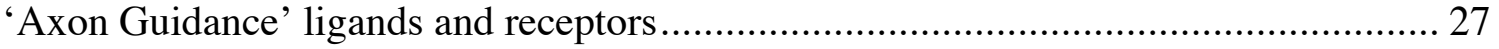

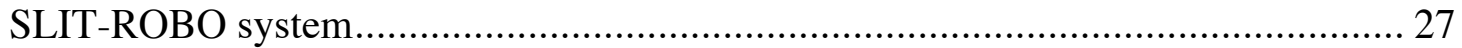

SEMAPHORIN-NEUROPHILIN / PLEXIN system ......................................... 28

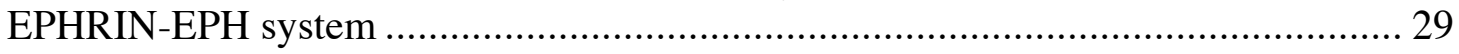

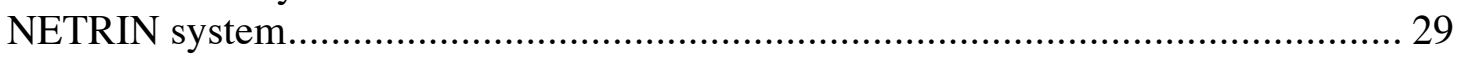

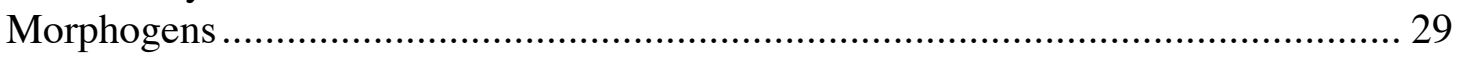

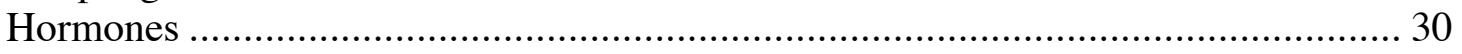

'Axon Guidance' mechanisms in the development of neocortex............................... 30

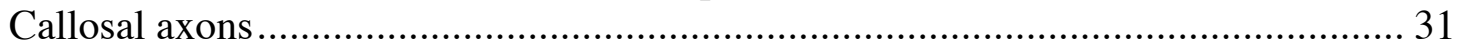

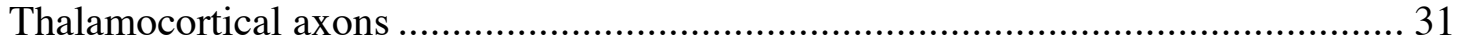

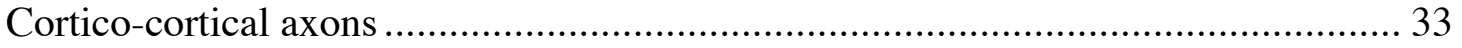

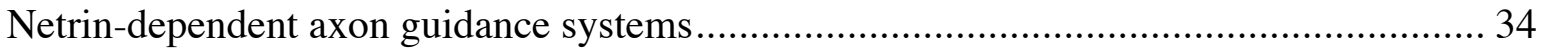

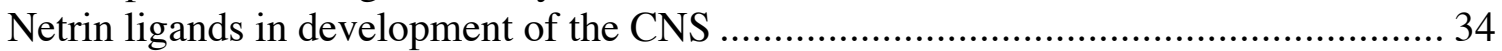

DCC family of receptors in development of CNS ................................................... 35

UNC5 family of receptors in development of CNS ............................................... 37

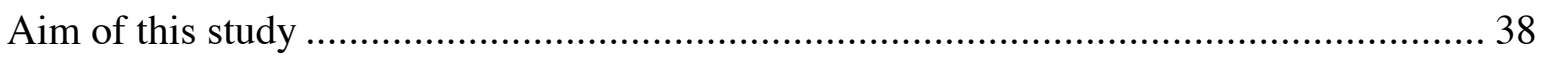


RESULTS

Determination of predominantly expressed genes in different cortical regions of E14.5

mouse embryos

In situ hybridization analysis of selected genes from the microarray data. 44

In silico identification of gene and protein structures 48

Identification of gene-structure of unc5A by RACE and RT-PCR Analysis 51

Generation of knockout constructs and ES-cell screens.... 54

Generation of chimaeras and screening for germline transmissions 61

General phenotypic investigations of unc5A knockout mice

Generation of 'Conditional Overexpression' construct and screening for founder animals 68

Creation of double transgenics with Cre-lines 71

Overexpression of UNC5A- $\Delta$ exon6 in developing neuroretina via ECG-Cre ................. 71

Overexpression of unc5A- $\Delta$ exon6 in Neocortex and Hippocampus via Nex-Cre ............. 78

DISCUSSION

Microarray analysis of E14.5 mouse cerebral cortices determined the sets of genes showing region-specific expressions among different cortical regions.

Expression analyses and in silico identification of candidate genes for functional studies. 89

Identification of unc5A gene structure and its spliced variants ..................................... 92

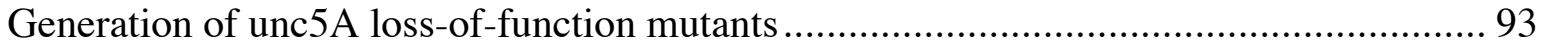

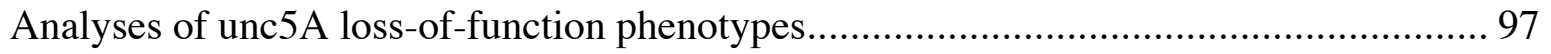

Analysis of unc5A gain-of-function mutants ............................................................. 100

Ectopic overexpression of unc5A- $\Delta$ exon6 in the embryonic distal neuroretina causes blindness in a background-dependent manner ..................................................... 102

Ectopic overexpression of unc5A- $\Delta$ exon6 in neocortex and hippocampus.................. 104

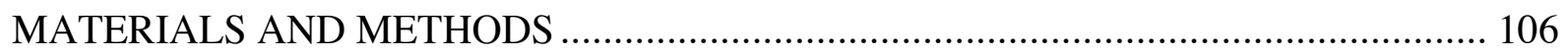

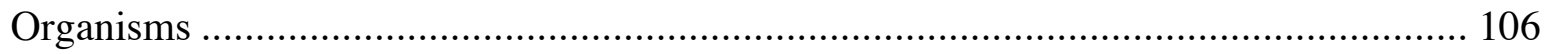

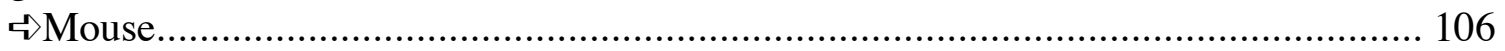

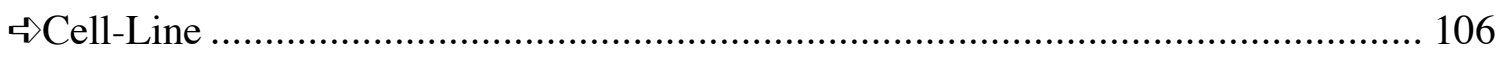

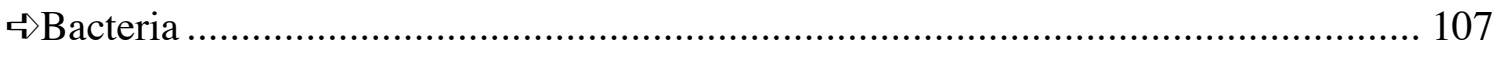

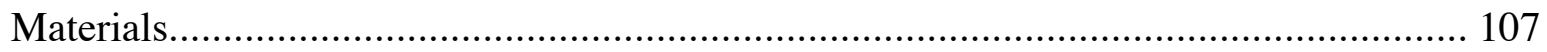

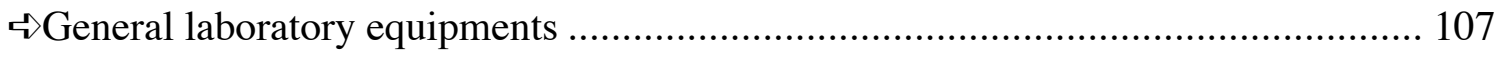

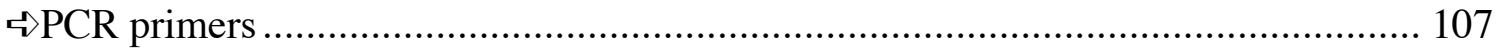

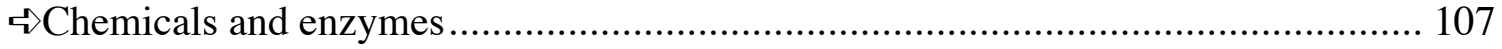

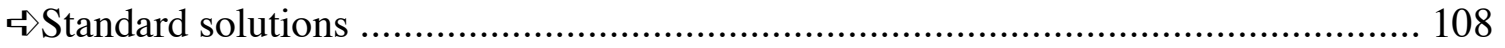

Basic molecular biology techniques .............................................................. 108

In silico gene-prediction studies and Bioinformatics ............................................... 109

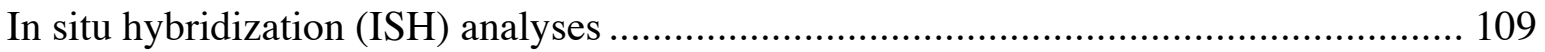

$\rightarrow$ Synthesis and purification of dig-labeled RNA probes ..................................... 109

$\nrightarrow$ Preparation of embryonic mouse brain sections ................................................. 111 


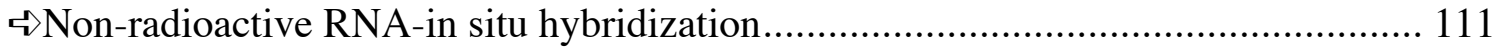

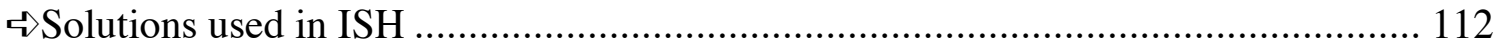

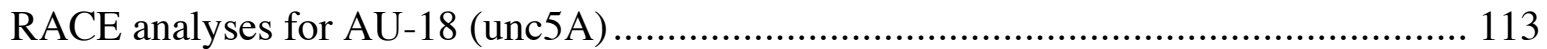

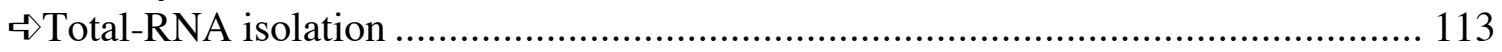

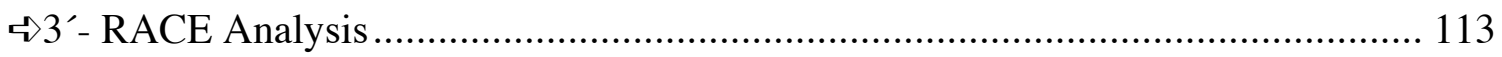

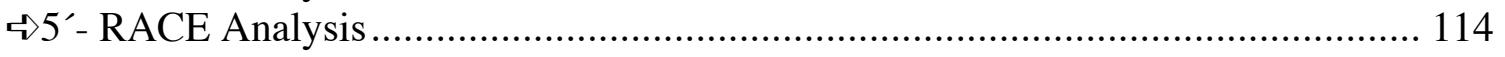

RT-PCR analyses for determining unc5A spliced variants and expression kinetics........ 114

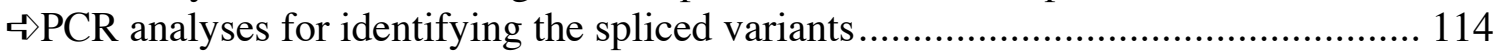

$\Rightarrow$ PCR analyses for identifying the expression kinetics ...................................... 115

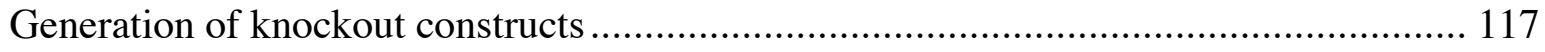

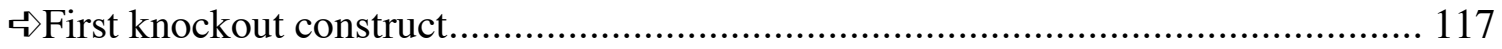

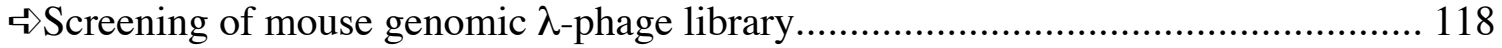

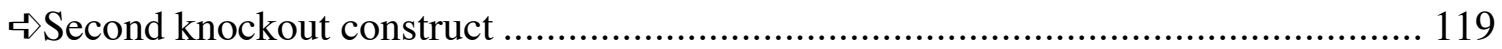

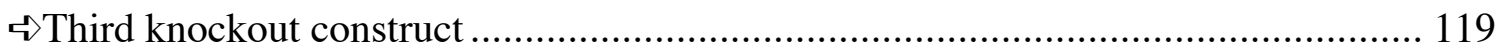

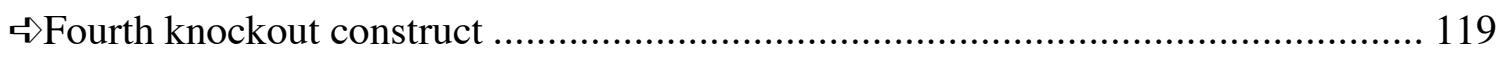

Southern-blot screening of ES cell clones for homologous recombination..................... 120

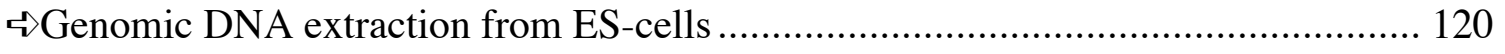

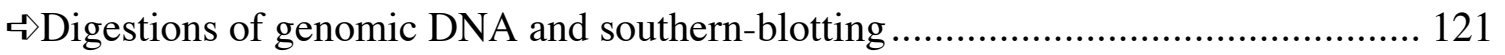

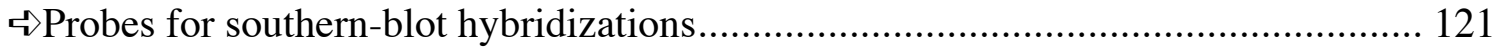

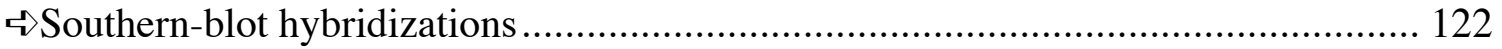

Generation of chimaeras and screening for germline transmissions ............................. 122

$\Rightarrow$ PCR-genotyping method for unc5A knockout mice …....................................... 123

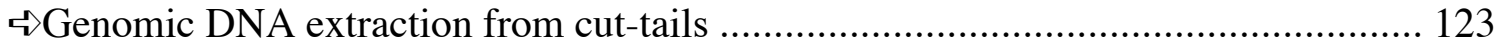

Investigation of general health and behavioral aspects of unc5A knockout mice ........... 124

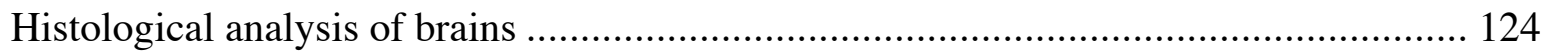

RT-PCR analysis for confirming the success of unc5A targeting construct................... 125

Generation of conditional-overexpression construct .................................................. 125

Generation of mouse lines for conditional-overexpression of UNC5A- $\Delta$ exon6 ............. 126

PCR genotypings of CRE- and reporter mouse lines .......................................... 127

Whole-mount LacZ stainings .............................................................................. 127

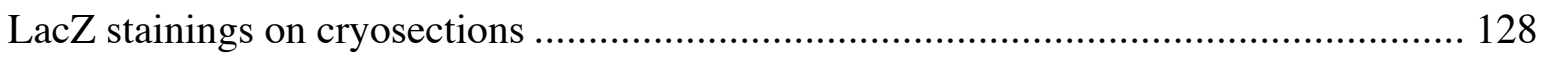

Transfection of Neuro2A cells and LacZ staining analyses ..................................... 128

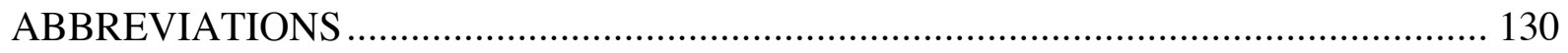

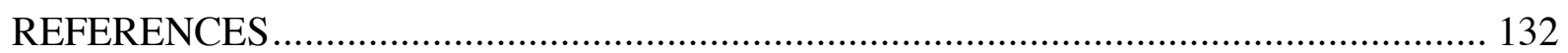

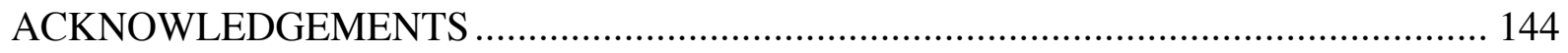

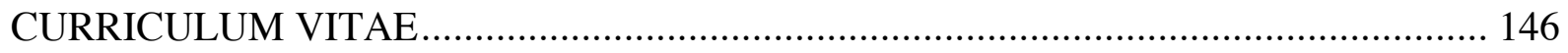




\section{ABSTRACT}

During the evolution of mammalian species, the cerebral neocortex acquired improved sensory, learning, and decision-making activities in parallel to its structural regionalization for specific functions. Each of these anatomically distinct neocortical areas is responsible for performing some different specific neural functions. However, their axonal connections with different neocortical or other brain regions are crucial for getting the necessary neural inputs and sending the output information. Therefore, elucidating the mechanisms responsible for the structural and functional development of the neocortex would provide a better understanding about its functional capacities as well as its developmental disorders in humans.

Regionalization and lamination of the neocortex are the two most important developmental processes taking place during the establishment of its structure. According to our current knowledge, the intrinsic 'protomap' mechanism (Rakic P., 1988 ) is responsible for the initial structural regionalization of neocortex. However, 'protocortex' mechanism (O'Leary DD., 1989) ensures the maintenance of this structure and establishes the functional regionalization via the thalamocortical axon (TCA) inputs. Therefore, it is important to identify and functionally characterize the genes that play important roles in each phase of neocortical regionalization. For this purpose, a large-scale comparative gene-expression analysis was done for E14.5 mouse neocortical regions by using the DNA microarray technology. This embryonic stage gives us the possibility of identifying new genes, which are responsible for early regional specificity as proposed by the intrinsic "protomap" hypothesis, since the TCA inputs from the dorsal thalamus reach the neocortex at stages later than E14.5.

Among the 216 region-specific genes for E14.5 dataset, a novel gene (unc5A) was selected for further analysis in order to identify and characterize its role during the neocortical regionalization. Unc5A encodes a chemorepulsive Netrin receptor and it is predominantly expressed not only in the visual and pyriform cortices, but also in the other brain structures important for the visual and olfactory systems. Netrin-dependent axon-guidance systems are important for the axonal path-findings and cellular migrations during CNS development. Therefore, we postulated that unc5A could play crucial roles during the functional and structural development of neocortex, especially 
for the visual and olfactory systems. In order to test these hypotheses, we created and analyzed the loss-of- and gain-of-function mouse mutants of unc5A.

The unc5A knockout mice are viable and fertile, showing that unc5A is dispensable for these two functions. Some of them are blind in a genetic-backgrounddependent manner. In 129 X CD1 mixed background, some unc $5 \mathrm{~A}^{-/}$mice are blind; whereas in pure 129 background, this phenotype is rescued by an unknown genetic trait of this strain. No gross abnormalities were detected in the brains of unc $5 \mathrm{~A}^{-/-}$mice in both mixed 129 X CD1 and pure 129 backgrounds. It will be necessary to backcross the unc5A knockout line into pure C57/BL6 background in order to efficiently analyze its phenotypes concerning the axon guidance deficiencies in the commissural pathway regions of the forebrain and neocortical regionalization.

Several transgenic mouse lines for conditional-overexpression of unc5A were also created during this study. Initially, unc5A was ectopically overexpressed in the distal neuroretina of the developing mouse embryos by aiming to disrupt the mechanisms responsible for the spatial representation of the neuroretina in the thalamus and the visual cortex. We observed blindness phenotypes in both unc5A overexpression -dependent and -independent manners due to the highly mixed genetic backgrounds of the hybrids. The latter case (overexpression-independent blindness) was successfully eliminated by partially backcrossing the Cre-line into C57/BL6-N background. Interestingly, the ratio of blind double transgenic hybrids (overexpression-dependent blindness) increased in parallel to the number of backcrossings of the unc5 $\mathrm{A}$ overexpression-line. In future, it will be important to reach a pure congenic state for the generated hybrids in order to have reproducible blindness phenotype and analyze its causes and also its effects on the visual cortex development.

The overexpression of unc5A was also established in the post-mitotic neurons of the neocortex and the pyramidal neurons of the hippocampus. It is possible with this system to identify the regions of neocortex where the netrin-dependent chemoattraction is important, and also to characterize the importance of 'switch on/off' mechanisms for the axons expressing unc5A endogenously. Since the double transgenic hybrids are healthy and fertile, they will allow us to study detailed behavioral and histological phenotypes in future. 


\section{ZUSAMMENFASSUNG}

Im Laufe der Evolution der Wirbeltiere entwickelte insbesondere der Neokortex spezialisierte, das heißt funktionell sowie anatomisch unterschiedliche Areale zur Verarbeitung sensorischer Informationen, zum Lernen sowie zu komplexen Prozessen wie entscheidungsorientiertem Verhalten.

Jedes dieser anatomisch unterschiedlichen Areale des Neokortex ist durch seine neuronalen Funktionen auf besondere Aufgaben spezialisiert. Darüber hinaus ist der Neokortex als übergeordnete Schaltstelle mit vielen anderen Bereichen des Gehirns vernetzt. Erst die Etablierung solcher komplexen neuronalen Netzwerke zwischen den verschiedenen Bereichen des Gehirns und somit den Stationen der Informationsaufnahme, -verarbeitung und weiterleitung ermöglicht den höheren Organismen die Bewältigung komplexer Aufgaben und die Entwicklung hoher kognitiver Fähigkeiten.

Aus diesem Grund stellt die Untersuchung und Aufklärung der molekularen Mechanismen der Gehirnentwicklung die Grundlage für ein besseres Verständnis der funktionellen Eigenschaften und Kapazitäten des Gehirns dar. Zugleich können solche Untersuchungen Ansätze zur Erforschung der Pathogenese und molekulare Grundlagen genetisch bedingter Krankheiten und Syndrome liefern.

Die zwei wichtigsten Prozesse während der Entwicklung der zellulären Architektur des Neokortex sind Regionalisierung und Laminierung. Nach einem aktuellen Modell der Kortexentwicklung, der so genannten Protomap-Hypothese (Rakic P., 1988), sind intrinsische genetische Mechanismen für die grundlegen destrukturelle Regionalisierung des Neokortex verantwortlich. Zum anderen scheint die Aufrechterhaltung und somit die spätere funktionelle Regionalisierung des Kortex von anderen Einflüssen, wie die Etablierung axonaler Kontakte von Neuronen aus dem Thalamus, abhängig zu sein (Protocortex-Modell, O’Leary DD., 1989). Die genauere Erforschung dieser Prozesse erfordert somit eine genaue funktionelle Analyse der Gene, die speziell für diese unterschiedlichen Entwicklungsphasen verantwortlich sind.

Aus diesem Grund wurde mittels der DNA-Microarray-Technologie eine großtechnische Genexpressionsanalyse mit embryonalem neokortikalem Gewebe (E14.5) durchgeführt, die die Genexpression in verschiedenen Kortexarealen miteinander vergleicht. Da der Kortex erst nach dem embryonalen 
Entwicklungsstadium E14.5 von thalamischen Axonen innerviert wird, war es möglich, selektiv Gene zu identifizieren, die nach dem Protokortex-Modell potentiell für die frühe intrinsische Regionalisierung des Neokortex verantwortlich sind.

Das zuvor unbekannte Gen unc5A wurde aus der Liste von 216 regionsspezifisch exprimierten Genen des Microarray-Datensatzes ausgewählt und auf seine mögliche Rolle während der Kortexentwicklung funktionell charakterisiert. Das unc5A-Gen kodiert einen Netrin-Rezeptor, der Chemorepulsion vermittelt und im Visuellen- und Piriformen-Kortex exprimiert ist, sowie in anderen Gehirnregionen, die für das Sehen und Riechen wichtig sind. Die durch Netrin vermittelten Systeme der Navigierung von Axonen sind während der Embryonalentwicklung des Zentralen Nervensystems (ZNS) für die Zielfindung und Migration dieser Zellen wichtig.

Aus diesem Grund wurde spekuliert, dass auch unc5A eine wichtige Rolle während der funktionellen und strukturellen Entwicklung neokortikaler Areale, insbesondere der Bereiche für das Sehen und Riechen, spielen könnte. Um diese Hypothese zu überprüfen, entwickelten und analysierten wir unc5A-Funktions-VerlustMutanten (loss-of function) sowie Funktions-Gewinn-Mutanten (gain-of function).

Unc5A-Funktions-Verlust-Mutanten sind lebensfähig und pflanzen sich normal fort, manche Individuen sind jedoch blind. Diese Blindheit scheint vom genetischen Hintergrund der Tiere abhängig zu sein, da nur Tiere in einem 129 X CD1 Hintergrund, nicht jedoch Tiere in einem reinen 129 Hintergrund, betroffen sind. Unabhängig vom genetischen Hintergrund wurde in unc5A Mutanten keine erkennbare phänotypische Veränderung des Gehirns festgestellt. Um eine weitere effiziente und reproduzierbare Analyse des Phänotyps bezüglich der kortikalen Regionalisierung und Zielfindung von Axonen der kortikalen Kommissuren durchführen zu können, ist es deshalb nötig, die Mutanten auf einen reinen C57/BL6 Hintergrund zurückzukreuzen.

Desweiteren wurden verschiedene transgene Mauslinien erstellt, die unc5A konditionell überexprimieren. Eine solche Überexpression von unc5A wurde zum einen in der distalen Neuroretina während der Embryonalentwicklung durchgeführt, um zu testen, ob dadurch die Entwicklung der örtlich festgelegten Projektion retinaler Axone in den Thalamus beeinträchtigt wird. Die Analyse zeigte, dass in transgenen Tieren unabhängig von der Überexpression Blindheit auftrat. Durch Rückkreuzung auf einen C57/BL6 Hintergrund konnte jedoch die überexpressions unabhängige Blindheit in transgenen Tieren überwunden werden. Interessanterweise erhöhte sich die Anzahl der 
überexpressions abhängigen blinden Individuen mit jedem Rückkreuzungsschritt. In Zukunft wird die Analyse der unc5A-Überexpression in einem reinen genetischen Hintergrund eine reproduzierbare Analyse der molekularen Grundlagen des BlindheitPhänotyps und die möglichen Effekte auf die Entwicklung des Visuellen Kortex ermöglichen.

Zusätzlich wurde unc5A in postmitoschischen und pyramidalen Neuronen des Neokortex überexprimiert. Diese transgenen Tiere stellen ein experimentelles Modell für die Untersuchung der kortikalen Areale dar, in denen Netrin-abhängige Mechanismen wichtig sind. Zusätzlich ermöglicht eine funktionelle Charakterisierung dieser transgenen Mäuse aufzuklären, ob sich das künstliche An- bzw. Ausschalten Netrin-abhängiger Mechanismen in Neuronen, die unc5A exprimieren, auf die Zielfindung ihrer Axone auswirkt. Da diese transgenen Tiere zudem lebens- und reproduktionsfähig sind, bieten sie ein gutes Modell zur weiteren Analyse des histologischen Phänotys sowie möglicher Verhaltensstörungen aufgrund möglicher Defekte der axonalen Navigation. 


\section{INTRODUCTION}

\section{Evolutionary view on brain and cerebral cortex}

3.4 billion years ago, when the first life form appeared on earth, it was just a unicellular organism. Actually for that step of evolution, it was the creation of a quite complex chemical factory, whose presence and existence was dependent on the simultaneous production of some thousands of different kinds of chemicals. It had to sense the environment through its membrane-associated sensors, interpret the input via receptors, which in turn would elicit a reaction as an output. For any life form since then, it is crucial to interact with the environment, and regulate the whole internal functions according to the conditions outside.

When 'Natural Selection' mechanisms on earth favored the first multicellular organisms, it required the specification of different cells for different functions that are necessary for the survival of the multicellular organism. The function of each individual cell was necessary for and dependent on the other cells' functions. But surely the need for sensing the environment, interpreting the sensory information, and then deciding for the response towards it remained as the most crucial feature of survival. These functions were carried out by the primitive neural and sensory (receptor) cells.

450 million years ago, when the first fishes appeared, they had a very simple brain structure. It was very small, but it was the earliest known ancestor of today's mammalian brain. 150 million years after that, when the first ancestors of the reptiles appeared, they had a more complex brain structure. Their brains had clearly divided into three compartments. The anterior part was responsible for 'smell', while the posterior part was for 'balance and coordination', and a compartment between these two regions controlled the 'vision'. The neural signals from receptors for vision and smell were coordinated in a part between 'smell brain' and 'vision brain', which was kind of a 'command post' center that is called diencephalon. The inputs from different senses were compared together here for the decision of the response. The basic instincts that are required for the survival of these reptiles, like sexual desire, search for food, and aggression responses of 'fight-or-flight', were controlled by this region of their brain. These brains of the reptiles were obviously inherited from the ancient forebear fishes and developed further according to the necessities of the organism. 
When the first mammals evolved from reptiles, 100 million years later, the structure of brains began to change in high magnitudes. These changes were mainly due to the appearance of new instincts and necessities of these mammalian species' survival. But since the instincts of ancient reptiles were still preserved in the further evolved mammals, the corresponding brain functions and structures have also been maintained in the mammalian brain. Further changes which have occurred during evolution includes addition of new parts for new instincts and functions into evolving mammalian brain while keeping old parts in their place. So the ' command post' center in the brain that controlled the instinctive behavior grew larger. The other big evolutionary change in ancient mammalian brain was the large expansion of their 'smell brain' while the 'vision' brain got partially diminished. This was mainly due to their nocturnal life style, since they had a 100 million years time of living in darkness because of the threat of ruling reptiles outside. So the sensation of smell got much more importance than vision for the survival of these species. Because of this, evolutionary mechanisms favored this specific modification in the brain compartmentalization. The two symmetric swellings in the 'smell brain' of these ancient mammalians' brain were the "cerebral hemispheres". Since the function of 'smell' was almost the only function for these cerebral hemispheres, the cranium of these ancient mammals was large enough in size to fit without wrinkling or folding (Kaas JH., 2004).

After the extinction of the ruling reptiles, mammals began to have a diurnal life style. This change brought back the importance of the visual sensation of the environment for their survival. So during the evolution of these newer mammalian species, the cerebral hemispheres got enlarged in size in order to create new circuits for getting information from eyes and analyzing them.

During the further evolution of mammalian species, the growth of cerebral cortex accelerated in order to create new functional domains, which were necessary for the newly acquired functions of those species. 120 thousand years ago, when the first Homo sapiens appeared, the evolved cortex already reached to such a massive size that the primitive region that held the circuits for the instinctive behavior of the reptile and old mammals -diencephalon-, was now completely enveloped by and buried within the human cerebral cortex. Furthermore, in order to gain more space for the evolutionary new circuits, it got many wrinklings and foldings. 
Relative to the body size, the human brain is bigger than any other animal brain. But this growth of size in evolving mammalian brain was not just simply doubling or tripling the old mammalian brains. The expansion or growth of different functional domains of cerebral cortex was differing according to evolutionary needs. There was also possibly the appearance of some new domains for new features or functions developed throughout evolution. For example, the responsible domains of cerebral cortex for learning and using languages have undergone a much higher extent of expansion during the evolutionary passage from old apes to humans, as compared to the growth of olfactory cortex. (Ponting C. and Jackson AP., 2005; Roth G. and Dicke U., 2005)

Although having a bigger brain or cerebral cortex looks like an evolutionary advantage, it also creates some problems to be solved by evolutionary mechanisms. First of all, it is the 'cost' of having a big brain. Among all organs, brain is the metabolically most expensive organ. Because of this reason, the expansion of brain during evolution occurred in parallel to the improvement or enrichment of diets of evolving species. Carnivores generally have much bigger relative brain size than herbivores, which is actually a bidirectional interaction. Carnivores need much more sensational, motor and social features in order to hunt instead of eating plants on lands. This need requires development of cerebral cortices, which are much more advanced in evolutionary terms. On the other hand, since they have a much better nutrient intake, they can afford having a bigger brain in terms of metabolic expenses. (Ponting C. and Jackson AP., 2005; Roth G. and Dicke U., 2005)

Second problem that can arise from having a bigger brain is the increased time it takes to get information from one place to another. The evolved solution for it was to do the things in brain locally. That means only the parts of the brain, which are functionally related and has to be connected are connected. So the need for communication between all parts of cerebral hemispheres was avoided in most, if not all, conditions. In the highly evolved mammalian brain, this problem has been solved by assigning different regions of the brain to have different functions. So the big brains of highly evolved mammals were compartmentalized into subdivisions for individual functions. (Ponting C. and Jackson AP., 2005; Roth G. and Dicke U., 2005) 
Considering that evolutionary mechanisms are working by natural selection of the newly created or modified genes, the genetic explanation of this expansion in brain size and its functional improvement in primate development is under several current investigations. For example, there are two important candidate genes, ASPM (abnormal spindle-like microcephaly associated) and microcephalin, which are actually causing the microcephaly in humans when they are mutated. At least for ASPM, it was shown that it plays a crucial role in mitotic spindle formation, which indicates that a change in its functional sequence would cause the decreased rate of cell division and hence brain size. (Ponting C. and Jackson AP., 2005). Another candidate gene is Nde1, whose deletion in mice results in having very small brains. (Sasaki S. et.al., 2005; Feng Y. and Walsh CA., 2004)

The GLUD2, which is a retrogene derived from GLUD1, first appeared 18-23 million years ago in hominoids. During integration into the genome as a retrogene, it picked up a brain-specific promoter. Over the next few million years, it underwent two important missense mutations. These amino acid changes made GLUD2 to be more stable and work more efficiently as a glutamate dehydrogenase compared to the GLUD1-encoded enzyme. Since GLUD activity is very important for recycling the neurotransmitter glutamate, this evolutionary change made hominoid brains to have greater neuronal activity than in monkey brains, which do not have GLUD2. (Burki F. and Kaessmann H., 2004)

In addition to the alteration of an existing gene by mutations or creating new genes based on what is already present, evolutionary mechanisms also worked on changing the spatio-temporal expression of some present genes by mutating some enhancer or regulatory sequences responsible for their expression. The expression patterns and timings of many homologues genes have some differences among human, chimps and macaques. It was also observed that many genes, which encode proteins important for metabolism, synaptic organization, and synaptic functions, are highly upregulated in human brains than in other tested primates. (Roth G. and Dicke U., 2005)

Altogether, these observations are providing clues to us in understanding how the 'new' brain (cerebral cortex) is evolved during the last several hundred million years of evolution from reptiles to humans. But unfortunately, all these evolutionary research is being focused on adult brain structures, instead of developing cerebral cortices. The main reason for this is our limited general knowledge about how the cerebral cortex is 
embryonically and postnatally developed. In the last 20-25 years, we are reaching to a better understanding of this crucial question, mainly in mice. The main reason of focusing this research on mice as the animal model is due to its being the easiest mammalian species to work on, availability of animals of well-defined genetic background and the improved transgenic technologies. But in comparative studies, which tries to interpret the data from mouse-research with medical observations on human diseases are also proving many similarities in developmental mechanisms of their cerebral cortex. (Molnar Z. et.al., 2006). So fully understanding the developmental mechanisms of cerebral cortex in a mammalian species, like mouse, would enhance our view of understanding the evolutionary mechanisms of creating human cerebral cortex. This will also give us a better understanding of many developmental diseases of the cerebral cortex.

In this regard, I will explain in the next sections the functional and morphological structure of mouse cerebral neocortex and its embryonic and postnatal development.

\section{Functional and morphological structures of cerebral neocortex}

\section{Cellular composition of cerebral neocortex}

Cerebral neocortex as being a neuronal tissue consists mainly of neurons and astrocytes. The neuronal cells of the neocortex are arranged in a perfectly ordered 6layered structure with specific cell types in individual layers with specific functions. These neurons can be categorized as (1) granular -also called stellate-, (2) fusiform, and (3) pyramidal neurons. (Guyton AC. and Hall JE., 1996; Paxinos G., 1985)

The granular neurons mainly function as intracortical interneurons with their short axons. There are mainly two classes of them: (i) Glutamergic interneurons, which release the excitatory neurotransmitter glutamate, and (ii) GABAergic interneurons, which release the inhibitory neurotransmitter gamma-aminobutyric acid (GABA). Both the sensory cortices and the association areas, which are located in between sensory and motor cortices, have large amounts of these granular cells. These cells are functionally very important for the high degree of intracortical processing of the incoming sensory 
signals in the sensory cortices and of the cognitive analytical signals in the association cortices. (Paxinos G., 1985; Guyton AC. and Hall JE., 1996)

The pyramidal and fusiform cells give rise to almost all the output fibers from the cortex. Pyramidal cells are much larger and much more numerous than the fusiform cells, and they are the source of very long and large nerve fibers that go all the way to the spinal cord. The large subcortical fiber bundles that pass from one major part of the brain to the other are also mainly the axons of these cells.

In almost any part of the cerebral cortex, one can see huge amounts of horizontal fibers that extend between adjacent areas of the cortex. There are also vertical fibers, which connect the cerebral cortex to ventral structures of the brain, spinal cord or other distant regions of the cortex in a bidirectional manner.

This 6-layered structure of cortex is maintained by placing different cell type compositions with specific functions in different individual layers. The order of the layers from the most outer to the deeper one are called as: Layer I (molecular layer), Layer II (external granular layer), Layer III (layer of pyramidal cells), Layer IV (internal granular layer), Layer V (large pyramidal cell layer), and Layer VI (layer of fusiform or polymorphic cells). (Paxinos G., 1985; Guyton AC. and Hall JE., 1996)

Most incoming specific sensory signals are terminating in Layer IV and most of the output signals leave the cortex from neurons located in Layers V and VI. Layers I, II, and III mainly perform most of the intracortical association functions via short horizontal connections with adjacent cortical areas.

The functions of Cerebral cortex are always performed in association with other brain regions, which are mainly based on extensive to-and-fro efferent and afferent connections of cortex with deeper regions of the brain. In this regard, thalamus is the most important collaborator of cortex. Thalamic excitation of the cortical regions is necessary for cortical functions. Also the development of cortex is very much dependent on thalamic inputs, as will be explained in the next section.

Every specific part of the cortex is connected with a specific nuclei of thalamus. These connections work in both directions; from thalamus to cortex, and then from the cortex back to essentially the same region of the thalamus. Because of this close association between cortex and thalamus, the term thalamo-cortical system is very frequently being used. Actually, all signal pathways from sensory organs to cortex pass through and include thalamus, with the single exception of olfactory system. According 
to this mechanism, the neuronal signals from sensory organs are brought to specific part of the thalamus, and they are transmitted from thalamus to specific cortical region via thalamocortical connections. (Paxinos G., 1985; Guyton AC. and Hall JE., 1996)

\section{Functional and morphological features of neocortical regions}

Cerebral cortex should be considered as an organ, which has morphologically and functionally distinct regions. This variety causes a huge heterogeneity (both at histological and functional levels) for cerebral neocortex that cannot be seen in any other organ of the body apart from brain. Here I will briefly describe many of these different neocortical regions based on their distinctive morphological structure and functions.

\section{Olfactory cortices}

In the most anterior part of the cerebral cortex, there are olfactory bulbs, which is an anterior outgrowth of cortical tissue in a bulbous enlargement structure. On the very anterior parts of the olfactory bulbs, there is cribriform plate, which separates brain cavity from upper reaches of nasal cavity. The axons of sensory neurons from the olfactory membrane in nasal cavity enter through cribriform plate into the olfactory bulbs. They end up in distinctive structures of olfactory bulbs, called glomeruli. It is believed that for each specific odor, there is a distinct glomeruli structure in the olfactory bulbs. Then the signals from glomerulis are transferred to two distinct cortical regions: Medial Olfactory area (MOA), and Lateral Olfactory area (LOA). These two areas located just on the posterior proximity of the olfactory bulbs. (Wilson DA. et.al., 2006; Rolls ET, 2004; Schoenbaum G. et.al., 2005; Guyton AC. and Hall JE., 1996; Paxinos G., 1985)

$M O A$ defines the evolutionarily very old olfactory system. It consists of several nuclei, so called septal nuclei. For each glomeruli in olfactory bulbs, there is an associated septal nuclei in MOA. These nuclei are associated with hypothalamus and other primitive portions of the brain's limbic system (Limbic system is the one which is concerned with basic behavior). This pathway of olfactory system are responsible for some primitive responses to olfaction such as licking the lips, salivation, and other 
feeding responses caused by the smell of food, or primitive emotional drives associated with smell, like response to pheromones. (Wilson DA. et.al., 2006; Rolls ET, 2004; Schoenbaum G. et.al., 2005; Guyton AC. and Hall JE., 1996)

LOA, on the other hand, comprises of evolutionarily newer olfactory system. It is mainly composed of prepyriform cortex, pyriform cortex and cortical portion of the amygdaloid nuclei. After the neural signal comes to these areas from olfactory bulbs, it passes to almost all portions of the limbic system, especially into the less primitive parts such as hippocampus. So this part of olfactory system is important for maintaining an olfactory memory, and giving responses according to previously obtained odormemory. (Wilson DA. et.al., 2006; Rolls ET, 2004; Schoenbaum G. et.al., 2005; Guyton AC. and Hall JE., 1996, Paxinos G., 1985)

\section{Motor cortices}

Primary and secondary motor cortices are located on the anterior half of the cerebrum, posterior to olfactory cortices and anterior to somatosensory cortices. It is responsible for "voluntary" movements of the body, which involves conscious activity controlled by the cerebral cortex. All the other motor activities are directed by spinal cord, cerebellum, and brain stem. But this does not mean that motor cortex can directly manage the conscious motor activity with direct connection to muscles. It still needs and use the pathway that involves basal ganglia, cerebellum, brain stem, and spinal cord. Almost all the skeletal muscles of the body are topographically well represented within both primary and secondary motor cortices, although representation ratio mainly depends on the complexity of any given motor activity.

The activity of motor cortex is mainly controlled by the somatic sensory system, but also in a lesser degree by auditory and visual systems. After getting the input from these sources, motor cortex functions in association with basal ganglia and cerebellum for creating the necessary motor action. The input signals reach to the motor cortex via: (1) subcortical fibers from ( $i$ ) adjacent somatosensory areas of parietal cortex, (ii) from adjacent frontal cortex, (iii) from visual and auditory cortices. (2) Subcortical fibers that pass through the corpus callosum from the opposite cerebral hemisphere. (3) Somatic sensory fibers from ventrobasal complex of the thalamus, which transmit cutaneous tactile signals and joint and muscle signals. (4) Fibers from ventrolateral and 
ventroanterior nuclei of the thalamus, which is actually the transmission of the signals coming from cerebellum and basal ganglia to these nuclei. (5) Fibers from the intralaminar nuclei of the thalamus. (Guyton AC. and Hall JE., 1996)

Output signals from motor cortex are transmitted to the spinal cord either directly through corticospinal tract or indirectly through multiple pathways, which involve the basal ganglia, cerebellum, and brain stem. Direct pathway is mainly concerned for discrete and detailed movements like in distal segments of the limbs.

Primary motor cortices contains a very special type of cells in Layer V, so called giant pyramidal cells or Betz cells, which produce very large myelinated fibers that are the most impressive fibers within the corticospinal tract. It is these cells, which maintain such a long distance connection between spinal cord and motor cortex. (Paxinos G., 1985; Guyton AC. and Hall JE., 1996)

\section{$\underline{\text { Somatosensory cortices }}$}

Somatosensory cortices (primary and secondary) are located in posterior to motor cortices, which are mostly on lateral faces of cerebrum. They are responsible for interpreting all the somatic senses. These somatic senses contain the neural information coming from all parts of the body. They can be grouped in three main categories: (1) mechanoreceptive somatic senses, which include both tactile and position sensations, (2) thermoreceptive senses, and (3) pain senses. (Guyton AC. and Hall JE., 1996)

Almost all somatic sensory information from the somatic segments of the body enters the spinal cord through the dorsal roots of the spinal nerves. Afterwards, these signals are carried via two different sensory pathways: (1) dorsal column-medial lemniscal system; in which the signal are mainly carried in the dorsal columns of spinal cord and then after synapsing and crossing to the opposite site in the medulla, they reach upwards to the thalamus (ventral posteriolateral and ventral posteriomedial nucleus) by the way of the medial lemniscus. (2) anterolateral system, in which the signals are crossing to the opposite site of the cord after being generated in the dorsal horns of the spinal gray matter, and ascend through the anterior and lateral white columns of the cord to terminate at all levels of brain stem and thalamus. From ventrobasal complex, which is the name of all nuclei of thalamus that receives all the somatic sensory inputs, third-order nerve fibers project mainly to the somatosensory 
area I. The distinct spatial orientation of all body parts through these spinal sensory pathways in thalamic nuclei, and finally in somatosensory cortices are well ordered and distinctly represented. The signals from one part of the body is never being mixed with signals from another part of the body.

Sensory signals from the taste receptors of tongue are also using the dorsal column-medial lemniscal system to reach ventral posterior medial nucleus of thalamus. Then just like any other somatic sensory signal, it is brought to the somatosensory cortex via third-order nerve fibers. (Paxinos G., 1985; Guyton AC. and Hall JE., 1996)

One of the very extensively studied issues of somatic sensation in rodents is the sensory system, which works via whiskers. For every whisker on the upper part of the lips, there is an associated structure in the thalamus (barrelettes), and also in the layer IV of somatosensory cortex (barrels). Layer IV is the region of the somatosensory cortex, where the inputs are received via thalamocortical axons from thalamus. This signal subsequently spreads towards the deeper and outer layers. The neurons in Layers II and III send axons to other related portions of the cerebral cortex, including to the opposite side of the cerebrum via corpus callosum. The neurons in Layer V and VI send axons to the deeper parts of the brain: From Layer V, very distant targets like basal ganglia, brain stem, and spinal cord are reached; and from Layer VI, axons generally reach to the thalamus back for providing feedback signals.

\section{$\underline{\text { Auditory cortices }}$}

The auditory cortex is located on the lateral side of the temporal cortex. Auditory signals reach from ears to the cerebrum via medulla and thalamus. More specifically, nerve fibers from the spiral ganglion of Corti enter dorsal and ventral cochlear nuclei, which is located in the upper portion of medulla. At this point, all these fibers synapse and second-order neurons pass mainly to the opposite side of brain stem and terminate in the superior olivary nucleus, although some of them also pass ipsilaterally to the superior olivary nucleus. From superior olivary nucleus, the nerve fibers pass upwards through the lateral lemniscus. Some of them terminate here, whereas majority of them bypass this nucleus and reach to the inferior colliculus, where almost all terminate. From this point, the pathway passes to the medial geniculate nucleus, where all the fibers again synapse. Finally, the pathway proceeds via auditory radiation to the 
auditory cortex. So in total of at least three points in the pathway from ear to cortex, crossing-over occurs: in the trapezoid body, in the region of Probst between two nuclei of lateral lemnisci, and in the region connecting two inferior colliculi. Apart from this pathway from ears to auditory cortices, there are also signal pathways from ears to brain stem, spinal cord, and cerebellum. Also for auditory system, the perfectly aligned spatial representation of the inner hair cells of cochlea within inferior colliculus, and within auditory cortices is maintained. The auditory cortex can be divided into two according to function and morphology: primary auditory cortex, and auditory association cortex (or secondary auditory cortex). Primary auditory cortices take their input signals from the auditory system mentioned above, whereas the secondary auditory cortices get their input signals from primary auditory cortices, and other regions of brain. (Paxinos G., 1985; Guyton AC. and Hall JE., 1996)

\section{$\underline{\text { Visual cortices }}$}

Both primary and secondary visual cortices are located in the most posterior region of the cerebrum. The visual pathway from both neuroretinas to visual cortex is one of the best-understood sensory systems at the molecular level. After nerve impulses leave the neuroretinas, they proceed backwards through optic nerves. At the optic chiasm, all the fibers from the nasal side of the retinas cross to the opposite side, where they get combined with the fibers from the temporal side of the opposite retinas to form the optic tracts. The fibers of each optic tract synapse in the dorsal lateral geniculate nucleus, and from there, the geniculocalcarine fibers pass through geniculocalcarine tract to the primary visual cortex in the occipital lobe. In the visual pathway, there are also signals going into suprachiasmatic nucleus of the hypothalamus, into pretectal nuclei, into superior colliculus, and into ventral lateral geniculate nucleus of thalamus.

The dorsal lateral geniculate nucleus, where all the optic nerves are terminating have a perfect spatial representation of the spatially distinct portions of neuroretinas. The similar spatial representation of neuroretina on this nucleus is transferred into the primary visual cortices, too. Just like in other sensory cortices, Layer IV of primary visual cortices get the input signals from retina via geniculocalcarine fibers, whereas secondary visual cortices gets their input signals from the primary visual cortices and other regions of brain. The optic signals, which are reaching to Layer IV of primary 
visual cortex, are then distributed to deeper and outer layers in the corresponding vertical columns. There are huge amounts of lateral signal transmission in outer layers (I, II, III). The signals from Layers V and VI pass long distances to the other visual cortices on the opposite cerebral hemisphere, to the thalamus back for feedback signaling, and also to the secondary visual cortices. (Paxinos G., 1985; Guyton AC. and Hall JE., 1996)

\section{Developmental aspects on functionality}

It therefore appears that cerebral cortex is a large heterogeneous tissue both in morphological and functional terms, although it mainly consists of the same or similar cell types. The main functional differences of different regions of neocortex can be explained mainly due to the fact that they make different synaptic connections. The area of thalamus and other brain regions that they are getting connected mainly defines their functional identity. Although they all share the same 6-layer cortical plate structure with similar cells in corresponding similar layers, there are some small morphological differences between them, too. Actually this is a long debate in the field of neocortex development if the functional thalamic connections are the driving force for the development of these morphological differences or vice versa. This will be explained in more detail within the next section of development of neocortex.

\section{Development of cerebral neocortex}

\section{Regionalization of cerebral neocortex}

During embryonic development, after the anterior neural tube closure the prospective brain consists in principle of just a sheet of pluripotent neuroepithelial cells. In order to develop a structure like cerebral neocortex, an extremely spatially heterogeneous organ, it is essential to create a 3-Dimensional frame. The dimensions will be considered as anterior-posterior axis, dorsal-ventral axis, and lateral-medial axis. There will also be a fourth "temporal" dimension, as it will be discussed in the frame of cortical development below. 
The mechanisms for creating the 3-D space information depend on the inductive signaling centers of forebrain development, which secrete morphogens. These forebrain inductive centers are: (1) Roof plate (Cortical Hem), which secretes BMP and WNT proteins, (2) Anterior neural ridge, which secretes FGF8 protein, (3) Prechordal mesendoderm (Medial Ganglionic Eminence), which secretes SHH protein. (O'Leary DD. and Nakagawa Y., 2002)

The secreted morphogenes being diffusible proteins, a concentration gradient is progressively established throughout the developing forebrain structures. The pluripotent progenitor cells of neuroepithelium sense the concentrations of various morphogens in their proximal environment and thus obtain clues for their spatial positioning in the developing brain. Subsequently, via mutual suppressive and/or enhancing effects of the downstream signaling cascades of the morphogen receptors, corresponding specific transcription factors and the specific differentiation mechanisms are activated. Some of the extensively studied and well-understood transcription factors in this mechanism are: (i) Pax6, which is expressed in anterio-lateral high, posteriomedial low gradient; (ii) Emx2, which has the complete opposite expression pattern as posterio-medial high, anterio-lateral low; (iii) Lhx2, which has a medial high, lateral low expression pattern. (Muzio L. and Mallamaci A. 2003). There is also an important dorsal-high, ventral-low expression gradient of Pax6. The expression of these transcription factors are directly regulated by the signaling cascades activated or suppressed by the morphogens from inductive signaling centers mentioned above. There are also other transcription factors, which are relatively less studied, like Emx1, Coup-TF 1, and Foxg1, whose expression are regulated via the above mentioned morphogen gradients. According to the combination and the magnitude of the expressed transcription factors in the progenitor cells of different regions of neocortex, different differentiation mechanisms are switched on. (Rubenstein JL. et.al., 1999; O’Leary DD. and Nakagawa Y., 2002).

Although such developmental system, which depends on the intrinsic factors, may function and look quite straightforward, many observations suggest that the "morphogenic identity determination" is not the only mechanism. In addition, input from the thalamocortical axons (TCA), which reach to specific regions of developing neocortex, is required. There was a long debate in the last decade between two hypotheses of neocortical development, which are: (1) protomap hypothesis (Rakic P., 
1988) suggesting that the regionalization of neocortex is already established before the arrival of TCA via the intrinsic morphogenic identity determination as explained above; (2) protocortex hypothesis (O'Leary DD., 1989) suggesting that the regionalization of neocortex is determined and maintained via the information input of the incoming TCA. Our current view on this topic combines both these hypotheses and proposes that the initial structural regionalization is established through the intrinsic "protomap" mechanism, however, the functional regionalization and the maintenance of structural regionalization depends on the TCA information flow, as suggested by the “protocortex” hypothesis. (Rubenstein JL. et.al. 1999; O’Leary DD. and Nakagawa Y., 2002). Therefore, the connections between developing neocortex and specific thalamic regions are crucial for the proper development of cerebral neocortex.

\section{Lamination of cortical plate in cerebral neocortex}

The mechanisms mentioned above explain only the structural regionalization of the developing neocortex, but not the origin of the 6-layered structures in any given region. The morphogen dependent mechanism cannot fully explain this aspect since almost all cells of each layer in any given cortical region are created from the same progenitor domain within the described 3D structure (with the exception of GABAergic interneurons, which are born in the ventricular and subventricular zone of basal ganglia and reaches to their final neocortical destinations via tangential migration). Actually, this is the point where the fourth dimension -time- is necessary as an additional factor.

The progenitors for all the neurons and astrocytes, which are produced in the ventricular zone (VZ) of developing neocortex, are the special cells called Radial Glia. These radial glia cells have long cytoplasmic protrusions which extends from the ventral to basal parts of developing neocortical tissue. The soma of these cells, which contain the nucleus, moves through these protrusions within the ventricular zone limits (basal and ventral) during the cell cycle. During the M-phase of the cell cycle, the cellular soma reaches the most ventral part, whereas during the S-phase they move away from the ventral to the basal side of the ventricular zone. The radial Glia cells can undergo two different types of mitotic division processes: Symmetric cellular divisions creating two identical sister cells which are both again radial glia cells or the asymmetric cellular divisions creating one radial glia cell and one post-mitotic neuron 
or neuronal progenitor cells of the subventricular zone. In the case of asymmetric cellular division, the post-mitotic neurons, which do not have the protrusions like radial glia cells, start to move through the developing cortical tissue by climbing over the protrusions of the radial glia cells. (Rakic P., 2003; Weissman T. et.al., 2003)

By the time window of E11-E12 of mouse development, the post-mitotic neurons created by asymmetric division move to the preplate and then to the cortical plate; whereas the neuronal progenitor cells created by asymmetric division move first to basal end of the ventricular zone or to the subventricular zone (SVZ) and undergo a further symmetric cellular division in order to create two neurons. Starting from E13, the neurons of cortical plate are being produced both from radial glia cells in the ventricular zone via asymmetric divisions and from neural progenitor cells in the subventricular zone via symmetric cellular divisions. All neurons created from both VZ and SVZ, migrate radially through outer layers of developing cortical plate with the aid of radial glia protrusions. This radial migration continues till they will reach the Marginal zone (MZ), which has a very specific extracellular matrix scaffold of Reelin proteins, secreted by Rajal-Cetzius cells. Then they will settle into the final location of their migration. The neurons that are born early will locate themselves on inner layers, and the later born neurons will settle on outer layers according to this mechanism, known as "Inside-first, Outside-last mechanism". Within this program of forming the cortical layers, S. McConnel observed that the transplantation of progenitor cells of G1phase from a developmentally younger neocortex into an older one will give rise to the adaptation of host system; whereas when the transplanted progenitor cell will be chosen from G2-Phase, then the created neurons will keep their layer identity (McConnell SK. and Kaznowski CE., 1991; McConnell SK., 1995). This suggests that the layer-identity of the post-mitotic neurons is determined during or after the DNA-Replication phase of their ancestral progenitor cells. So the final commitment of neurons for different layers is starting not when they are settled in the cortical plate but instead even before they are born. That means, what makes the neurons of different layers distinct, although they are born from the same pool of progenitor cells, is the developmental time of their birth. This can be easily explained with the spatio-temporally changing activities of signaling mechanisms, and spatio-temporally changing expressions of transcription factors in $\mathrm{VZ}$, and SVZ. (Rakic P., 2003; Weissman T. et.al., 2003) 
So from a simple sheet of neuroepithelium, the neocortex is being structurally developed based on spatial cues for the regionally distinct structure, and temporal cues for the cellular distinctions of layers in each different regions. But one should also consider that apart from spatial and temporal cues explained above, the TCAs and their synapses with high specificity to distinct regions of neocortex and to cells of distinct layers in each neocortical regions are crucial at least for fine-tuning and maintenance of the developing neocortex.

Although we have satisfactory -but not sufficient- information about how the neocortex structure is developed, we still do not know much about how the neocortex is developed in functional terms. Surely, just having a perfectly designed and established structure of neocortex is not enough for understanding its importance. With the mechanisms that are explained above we can think of creating the highly specific and differentiated neurons into the correct locations within neocortex. But what is equally important is to understand how they will later on create the perfect neural networks via creating synapses with the correct partners adjacent to them, and also very far away from them. So how neocortex is developed for maintaining its functionality is the big question waiting to be asked and investigated. In this sense, the role of axon guidance systems and synaptic mechanisms has to be clarified further in the development of a functioning neocortex.

\section{$\underline{\text { Axon guidance systems in cerebral neocortex development }}$}

After generation in the ventricular zones, neurons migrate to their final destinations and differentiate into their final state. However, their final locations are often very distant from their synaptic target cells. They establish these connections via extending long axons, which have special sensing devices on their tips called growth cones, as it was first named in 1880 by Santiago Ramon y Cajal. Growth cones sense and interact with the molecules within the environment that they are growing through. These "axon guidance" molecules attract or repel the growing axons via interacting with their corresponding receptors on the surface of the growth cones. To date, we know several families of "axon guidance" molecules, which can be grouped into two classes. The first group contains the membrane-bound molecules that act for short-range chemoattraction or chemo-repulsion. Second group consists of secreted molecules in the 
extracellular matrix and act as long-range chemo-attractants, or chemo-repellants. The spatially and temporally regulated expression of these axon guidance molecules enables the growing axons to navigate within the complex structure of brain. There is also temporal regulation of many axon guidance receptors within specific neural axons, and this will make them to respond to specific axon guidance molecules in the correct time and correct location of their paths. When they reach their targets at the end of the pathfinding, they stop growing and establish the synaptic connection with the target cell. (Mueller BK., 1999; Tessier-Lavigne M. and Goodman CS., 1996; Plachez C. and Richards LJ., 2005; Chilton JK., 2006)

\section{Molecular mechanisms of 'Axon Guidance'}

The receptors for these axon guidance molecules within the growth cones directly or indirectly regulate many different types of actin-associated proteins depending on their interaction with the axon guidance ligands. Thus, they regulate the structure and dynamics of the actin-cytoskeleton of the growing axon in order to cause attraction, repulsion or collapsing. (Plachez C. and Richards LJ., 2005; Chilton JK., 2006; Dent EW. and Gertler FB., 2003)

Axon outgrowth occurs in three steps: protrusion, engorgement and consolidation. Protrusion of the growth cone occurs by rapid extension of fillopodia and thin lamellar extension in the growth cone while the cytoplasmic membrane advances forward. During engorgement, the organelles and cytoplasm move forward. Finally in consolidation, axon outgrowth is being accomplished by advancing the growth cone further. The cytoplasm contains neurofilaments, which provide the structural organization, and microtubules, which provide a scaffold for rapid vesicle transport. This vesicle transport is crucial to maintain and re-organize the membrane as it changes. (Goldberg DJ. and Burmeister DW., 1986; Plachez C. and Richards LJ., 2005; Dent EW. and Gertler FB., 2003; Goldberg DJ. and Burmeister DW., 1989; Chilton JK., 2006)

Growth cone turning is essential for almost all axonal navigations and is responsible for changing the direction of neurite elongation. When a growth cone contacts a positive guidance cue (chemo-attractive), fillopodia become stabilized in the neurite elongation direction and microtubules are locally reoriented in order to advance 
toward the contact site. (Bentley D. and O'Connor TP., 1994; Sabry JH. et.al., 1991). But when the growth cone gets a negative cue (chemo-repulsive), actin filaments and microtubules of growth cones may interact in order to accomplish turning away from the site of cue or stopping the extension completely in a process called "growth cone collapse". The collapsing will occur with the loss of all lamellipodia and fillopodia within the growth cone in response to a negative cue. Actually the growth cone turning occurs with asymmetric growth cone collapsing. The site of the growth cone close to the repulsive cue will collapse, whereas the site away from the cue will continue to extend. (Plachez C. and Richards LJ., 2005). The exact molecular mechanisms for growth cone collapse and growth cone turning in response to a negative cue is not fully understood yet. It is believed that endocytosis which occurs in the growth cones near chemo-repulsive signals is important for the process (Fournier AE. et.al., 2000; Chilton JK., 2006). But the main link of chemorepulsion with cytoskeletal changes is waiting to be revealed.

\section{Different strategies of 'Axon Guidance' in neural systems}

In neural systems, the connections between neurons of two distant regions are established during development with the elaborate use of 'pioneering axons', intermediate targets of axon pathfinding and the use of 'Glial Guidepost' cells. (Plachez C. and Richards LJ., 2005; Chilton JK., 2006)

Before a big bundle of nerve axons establish their connections, generally some small set of 'pioneering axons' are already creating the 'path', which will later on guide the main sets of axons in order to follow through. In the neocortex, subplate neurons have been shown to serve as pioneering axons for thalamocortical and corticothalamic axons (De Carlos JA. and O'Leary DD., 1992; Ghosh A and Satz CJ., 1992; Ghosh A. and Satz CJ., 1993). Also in the medial cortical projection, the first axons that cross the rostral cortical midline are derived from neurons in the cingulate cortex. Then they are followed by neocortical axons, which mainly grow within the tract of pioneering cingulate cortex axons, and possibly fasciculating with them (Rash BG. and Richards LJ., 2001).

When one considers the long path of axons in brain, one very common observation is that they extend in one direction up to a point, and then they turn and start to extend in a different direction. This is very important to create the efficiently 
functioning neural networks in brain, and it mainly depends on usage of intermediate targets during axonal pathfinding. Pioneering axons are one set of these intermediate targets, whereas others are 'Glial Guidepost' cells and structures present along the pathway that secretes different guidance factors. Once a growth cone reaches such an intermediate target, it slows down and changes its morphology in order to look for further molecular direction cues. (Plachez C. and Richards LJ., 2005; Chilton JK., 2006)

Guidepost glial cells, which was first identified in Drosophila (Auld V., 1999), secrete guidance cues and also express cellular cues on their surface that guide axonal outgrowth. Similar mechanisms of guidepost glial cells are found in vertebrates during the development of spinal cord, the ventral roots, the optic nerve, the auditory system, and the corpus callosum. During the embryonic development of CNS, these cells are vital in order to define boundaries between different brain areas or between functional subdomains within the same area. These glial boundaries act in order to prevent axons from straying from their correct elongation path. (Plachez C. and Richards LJ., 2005; Chilton JK., 2006)

\section{'Axon Guidance' ligands and receptors}

Most axon guidance molecules were first discovered in Drosophila and C.elegans via random mutagenesis screenings. There are four main classes of axon guidance molecules and their receptors, which were identified in both invertebrates and vertebrates. These are: Slits, Semaphorins, Ephrins, and Netrins as guidance molecules and their corresponding receptors within the growth cone of elongating axons. Apart from these four classes of molecules, it was also shown that a number of other molecules, like morphogens, steroids, extracellular matrix proteins and cellular adhesion molecules could function in guiding the axons. (Plachez C. and Richards LJ., 2005; Chilton JK., 2006)

\section{SLIT-ROBO system}

Slit family of proteins are multifunctional guidance cues with roles in regulating neuronal migration (Wu W. et.al, 1999), axonal and dendrite branching (Ozdinler PH. and Erzurumlu RS., 2002), and axon guidance (Brose K. and Tessier-Lavigne M., 
2000). There are three members of this family of large glycoproteins: Slit1, Slit2 and Slit3. They have been identified both in developing and adult mammalian CNS. They all share three types of protein motifs: leucine-rich repeats, EGF-like repeats, and a laminin G domain. Loss-of-function studies of Slit1 and Slit2 showed that they play important roles in the establishment of several mammalian fiber tracts, including corticofugal, thalamocortical, callosal, optic and lateral olfactory tract. (Bagri A. et.al., 2002)

The Robo family of transmembrane proteins are the receptors for Slits. They are the members of the immunoglobulin superfamily of proteins. There are three identified mammalian homologs: Robo1, Robo2, and Rig-1. Their extracellular domains consist of five IG-like repeats, and three Fibronectin type III repeats (Kidd T. et.al., 1998).

\section{SEMAPHORIN-NEUROPHILIN / PLEXIN system}

Semaphorin family of guidance proteins contains both secreted and membranebound members, divided into eight classes: Class 1 and 2 represent invertebrate semaphorins; Class 3-7 represent vertebrate members; and class 8 is the viral semaphorin. Among the vertebrate classes, class 3 semaphorins are secreted proteins, whereas others are either transmembrane proteins or membrane-anchored molecules. All members of the family share a 500 amino acid-long sema domain. (Kolodkin AL. et.al., 1993)

There are two main classes of semaphorin receptor families: Neurophilins (two members: Npn1 and Npn2), and Plexins (nine members: PlexinA1-4; PlexinB1-3; PlexinC1; and PlexinD1). (Fujisawa H. and Kitsukawa T., 1998) They usually form multimeric -complexes composed of Semaphorin ligands, several of Neurophilin and Plexin Receptors, and also some extracellular matrix (ECM) and cell adhesion molecules. Chemo-repulsive or -attractive responses for each Semaphorin ligand is determined according to which Semaphorin receptor and adhesion or ECM protein are forming these multimeric complexes. (Plachez C. and Richards LJ., 2005; Chilton JK., 2006) 


\section{EPHRIN-EPH system}

Ephrin family of guidance proteins are grouped into two classes: Ephrin-As (5 members: A1-5) are anchored to the membrane via glycosyl-phosphatidyl-inositol (GPI) linkage, whereas Ephrin-Bs (3 members: B1-B3) are transmembrane proteins. Eph receptors, which are tyrosine kinase receptors for Ephrin ligands, are divided into two subgroups according to what type of ligand they bind: 'A' subclass contains eight members (EphA1- EphA8), which bind to most or all types of Ephrin-As; whereas 'B' subclass contains five members (EphB1- EphB4, and EphB6), which bind to most or all types of Ephrin-Bs. The only exception for this rule is EphA4, which can bind to almost all Ephrin-As and Ephrin-Bs. (Huot J., 2004; Kullander K. and Klein R., 2002).

Since both the ligand (Ephrin) and receptor (Eph) are bound to the membrane, their interactions require intracellular contact; and they can act bidirectional, which means that a ligand can act as a receptor and a receptor also can act as a ligand (Bruckner K. et.al., 1997; Holland SJ. et.al., 1996). This Ephrin-Eph receptor system regulates many cellular functions based on cytoskeletal remodeling, such as axon guidance and synaptic plasticity. The interactions are generally of repulsive character, although there are also examples where an adhesion or attraction is the final outcome. They are shown to have important functional roles in the development of spinal cord motor axons, retinal axons, and anterior/posterior tectal/superior collicular axes. (Huot J., 2004; Feldheim DA. et.al., 2000; Hu Z. et.al, 2003; Plachez C. and Richards LJ., 2005; Chilton JK., 2006)

\section{NETRIN system}

Netrins and their receptors will be discussed in more detailed way in the next sections.

\section{Morphogens}

Morphogens are best known for their roles in the mechanism of cell fate determination during development. Many recent studies stated that many of these morphogens, like $W N T$ family, $B M P$ family, and Shh family are also playing important 
roles in axon guidance. For example, the Loss-of-function of Frizzled-3, a WNT receptor, cause severe defects in several major axonal tracts in the vertebrate forebrain (Wang Y. et.al., 2002). WNT proteins are shown to regulate terminal arborization and synaptic remodeling within the developing CNS (Hall AC. et.al., 2000). There are also many indications for the role of WNT pathways in axon guidance coming from genetic studies in Drosophila. It is also shown that BMP morphogens regulate the chemorepellent activity of the roof plate during spinal cord development (Augsburger A. et.al., 1999). The defects in retinal ganglionic cell projections in mice with deletion of a BMP receptor, BMPR-1B, also provide another example for the involvement of morphogens in axon guidance.

\section{Hormones}

There are steroid hormones, which induce neurite outgrowth and indeed axon guidance. For example, Estrogen enhances the neurite outgrowth and differentiation in organotypic explant cultures of hypothalamus, preoptic area, and neocortex. (ToranAllerand CD., 2004).

So, actually any molecule that has an influence on certain actin regulatorysignaling cascades, such as Ena/VASP pathway or RhoGTPase pathway within the axons, will play a role in axon guidance. In this sense, many extracellular matrix proteins, like Laminin, tenascin, collagen, fibronectin, and a number of proteoglycans; and cell adhesion molecules, like LICAM, NrCAM, and TAG-1, were also shown to regulate the axon guidance in many parts of the neural development. (Plachez C. and Richards LJ., 2005; Chilton JK., 2006)

\section{'Axon Guidance' mechanisms in the development of neocortex}

The extremely complex nature of the mechanisms for regulating the axon guidance ensures the establishment of the correct synaptic connections. At this point, I will focus on what is known about the regulation of axon guidance during neocortical development and give a brief review of the current understandings. The most studied models for axon guidance in neocortex development are the ones for callosal axons, corticocortical axons, and thalamocortical axons. 


\section{Callosal axons}

Contralateral cerebral cortical projections through the corpus callosum are important for integrating the sensory and motor information between two cerebral hemispheres. All the current data in humans and in mice suggest that different combinations of mechanisms are regulating the development of corpus callosum across its anterior-posterior extent. During development, the callosal axons from neocortical layers 2,3 , and 5 grow ventrally to the intermediate zone, and then turn medially, cross the midline, and re-enter the appropriate contralateral cortical area to form synaptic connections with their targets. Pioneering axons in cingulate cortex, and the midline glial cell populations are functionally important for this process. Slit proteins secreted by these glial cells, and ROBO receptors in the callosal axons are shown to regulate this axon guidance event at least in some regions of neocortex. Also Netrin-DCC guidance mechanism is crucial for the regulation of their axon guidance. Netrin-1 mutants and DCC-deficient mice do not develop corpus callosum or a hippocampal commissure, and they have a greatly reduced anterior commissure. (Plachez C. and Richards LJ., 2005; Price DJ. et.al., 2006)

The deletion of L1CAM cell-adhesion molecule also results in failure of corpus callosum development in mice. The other genes which cause a failure or defect in corpus callosum when they are knocked-out are: Nuclear factor 1 (Nfi1); p35 - an activator of cdk5-; Ephrin receptors EphA5, EphB2, and EphB3; a microtubule associated protein MAP1B and many others. (Plachez C. and Richards LJ., 2005; Price DJ. et.al., 2006)

\section{Thalamocortical axons}

As it was mentioned in previous sections, most of the signal inputs to the cerebral cortex come via the thalamus from thalamocortical pathway. During the development of neocortex, the early establishment of this pathway is achieved by the growth cones of thalamic neurons navigating first ventrally through the prethalamus, and then laterally into the ventral telencephalon and finally dorsally towards the cerebral neocortex. This typical outgrowth of thalamic axons is directed by several molecular cues between the 
thalamus and neocortex. The transcription factors, Pax6 and Gbx2 are vital for thalamocortical pathway establishment. (Hevner RF. et.al., 2002) Gbx2 has a restricted expression in the thalamus, whereas Pax6 is expressed in the thalamus, along the pathway of TCA and also in neocortex. From many experimental results, it was postulated that both Pax6 and Gbx 2 regulates the expression of downstream molecules that are necessary for the TCA growth cone's ability to navigate. However these downstream molecules are not fully characterized yet. One possible system regulated by Pax6 would be cadherins, since the cell adhesion is an important regulator for axonal guidance. Other candidates are Sema3C and Sema5A, whose expressions are regulated by Pax6. (Molnar Z. and Blakemore C., 1995; Innocenti GM. and Price DJ., 2005; Price DJ. et.al., 2006)

In the long path that TCAs are navigating, they pass through and across several anatomical and gene-expression boundaries, where they change their kinetics and fasciculation. Two of the well-understood critical zones within the path are 'diencephalic-telencephalic border', and 'pallial-subpallial boundary'. It is shown that, when the diencephalic-telencephalic border is displaced (in Emx2 mutant) or missing (in Mash1 mutant, Foxg1 mutant or Pax6 mutant), the TCA either derailed while they are exiting or are not even exiting at all. For pallial-subpallial border, a pioneering axon mechanism is important. Before the TCA reaches this border region, the early corticofugal projections reach and cross the border. According to the 'handshake hypothesis', these axons of early born cortical preplate cells meet with the growing TCA in the basal telencephalon and then TCA uses the tract of these corticofugal projections in order to pass the pallial-subpallial border and finally to reach the final destination within the cortical plate. Concerning all these processes, very little is known about the directly involved axon guidance molecules. There are evidences that show limbic-associated membrane protein (LAMP), cadherins, ephrins, Eph receptors, neurotrophins, netrin-1, and semaphorins are playing a role in TCA guiding. The lossof-function of corresponding genes generally give subtle phenotypes instead of a severe guidance defect. This can be explained either by each of them playing a minor role individually, or by them all acting together. So the lack of one is mostly compensated by the presence of all others. To date the most drastic phenotypes for TCA guidance are observed in mice with mutations in Celsr3, a gene coding for a protocadherin, and Fzd3, a member of the Frizzled family that is encoding for a WNT receptor. In both of these 
mutant mice, the anterior commissure and internal capsule is absent and no fiber bundle crossing the striatum is present. (Molnar Z. and Blakemore C., 1995; Innocenti GM. and Price DJ., 2005; Price DJ. et.al., 2006)

The TCA from different nuclei of thalamus are specifically connecting to specific cortical regions. This is one of the very important features of TCA growth in cortical regionalization. How this orderly fashioned alignment of TCA is obtained is an important question still waiting to be clarified. The only clues we have by now are the involvement of Ephrin and Eph receptors. It has been partially shown that Ephrindependent mechanisms are responsible for sorting the TCA to control the topography in ventral telencephalon. (Molnar Z. and Blakemore C., 1995; Innocenti GM. and Price DJ., 2005; Price DJ. et.al., 2006)

It has also been postulated that the neural activity plays an important function in the final establishment of functional neocortex topography. There are several studies, which explains that TCA growth is terminating to create the synapses with Layer 4 interneurons in a mechanism dependent on the neural activity. But still the core of knowledge in order to explain these phenomena is unclear. (Molnar Z. and Blakemore C., 1995; Innocenti GM. and Price DJ., 2005; Price DJ. et.al., 2006)

\section{Cortico-cortical axons}

The cortico-cortical axons are responsible for connecting the cortical regions on the two sides of the cerebral hemispheres and also on the same side of the brain ipsilaterally. For the development of these corticocortical axons, the main strategy is overproduction of this type of axons, and then selecting the correct ones and eliminating the rest. It is highly possible that the same molecules and transcriptional regulators, which are responsible for the guidance of TCA within neocortex, are also responsible for guidance of corticocortical axons. But unfortunately, we have no clear information about the exact molecular mechanisms that are responsible for guiding these corticocortical axons and also for selective elimination of the unnecessary or wrong ones. (Innocenti GM. and Price DJ., 2005; Price DJ. et.al., 2006) 


\section{Netrin-dependent axon guidance systems}

Netrin-1 protein acquired its name from the Sanskrit term meaning 'one who guides' ("neta"=leader). It was originally purified in vertebrates as a floor plate-derived chemoattractant in a large-scale purification from over 10,000 chick brains that can promote commissural axon outgrowth (Kennedy TE., 1994; Serafini T., 1994). There are several members of Netrin family, which were identified in a variety of species: the unc-6 gene product in nematodes; netrin-A and netrin-B in Drosophila; netrin-1 and netrin-2 in zebrafish and chicken; netrin-1, netrin-3, netrin-G1, netrin-G2 and netrin-4 ( $\beta$-netrin) in mice. These genes encode approximately $60-80 \mathrm{kDa}$ secreted proteins that share homologous domains with Laminin, especially the EGF-like repeat motifs. In functional terms, they are bidirectional axon guidance molecules, which means that they act as chemoattractants or as chemorepellants according to the receptors on target cells or axons. Chemo attractive response to Netrins is mediated by DCC family of receptors, whereas UNC5-family of netrin receptors are responsible for chemorepulsive response to Netrins. (Barallobre MJ. et.al., 2005; Plachez C. and Richards LJ., 2005)

\section{Netrin ligands in development of the CNS}

Netrin-1 is the most studied member of the family. In all the analyzed vertebrates, it is expressed in the floor plate and specifically in the neuroepithelial cells of the ventral part of the spinal cord during development. In the developing brain, it is mainly expressed in the midline and ventral ventricular zone; but also in other structures like ganglionic eminence, internal capsule, the fimbria, external germinal layer of cerebellum, and in the lateral septum. Apart from CNS, its expression during development was also shown in the limb-primordium, mammary gland, somatic mesoderm, dorsal aorta, cardiac muscle, and pancreas. In adult, it continues to be expressed in high levels by spinal cord neurons and oligodendrocytes, and also in ovaries. (Barallobre MJ. et.al., 2005; Plachez C. and Richards LJ., 2005)

Netrin-1-deficient mice lack the corpus callosum, the hippocampal commissure, and the anterior commissure (Serafini T. et.al., 1996). This indicates that Netrin-1 is vital for axon guidance towards the midline, and also at more rostral levels. There are also severe defects in the projection of commissural axons of the spinal cord. Netrin-1 
signaling is also crucial for proper TCA guidance in developing brain (Braisted JE. et.al., 2000). Another phenotype in this mouse is that the retinal ganglionic cell axons cannot exit to the optic nerve, which results in optic nerve hypoplasia (Deiner MS. et.al., 1997). In the development of hippocampus, and cerebellum, many severe deficiencies were also observed in these mice (Barallobre MJ. et.al., 2000). There are also several evidences in vitro and in vivo, which indicate a role for Netrin-1 in guiding cellular migrations of dopaminergic neurons in the midbrain, cerebellar granule cells, some specific cells of hypothalamus, and many others. Also in the morphogenesis of semicircular channels in the inner ear, mammary gland, pancreas, and lung, the functional importance of Netrin-1 was shown. (Barallobre MJ. et.al., 2005; Plachez C. and Richards LJ., 2005)

Netrin-3 expression is shown partially in late embryonic thalamus, and motor columns in ventral spinal cord. But the role of Netrin-3 in any developmental mechanism is unclear yet. (Barallobre MJ. et.al., 2005)

Netrin-4 is expressed in basal membranes of several embryonic and adult tissues. Its high expression in retina, and olfactory bulbs, and low expression in the embryonic spinal cord has been shown. In the postnatal brain, it is expressed in cerebellar granule cells, and in pyramidal cells of the hippocampus. Its functional role in CNS development is still unclear, although it is believed to play important roles in angiogenesis during vascular system development. (Barallobre MJ. et.al., 2005)

Netrin-G subfamily, which contains two members, is characterized by their binding to the cellular membranes via glycosyl-phophatidyl-inositol. None of these two members bind to DCC or UNC5 family of Netrin receptors. They have wide expression patterns in the developing CNS, but their functional roles in development are not well understood. (Barallobre MJ. et.al., 2005)

\section{DCC family of receptors in development of CNS}

DCC family of chemoattractive receptors for netrin ligands consist of DCC and Neogenin in vertebrates; UNC-40 in nematodes; and Frazzled in Drosophila. They belong the immunoglobulin superfamily of proteins and have a transmembrane domain, which crosses the membrane once. They share sequence similarity with NCAM. They contain four immunoglobulin domains together with six type III Fibronectin repeats in 
the extracellular domain; a transmembrane domain; and a cytoplasmic domain, which has no similarity with any other known proteins. Although they are known as chemoattractive Netrin receptors, they also play a role in Netrin dependent chemorepulsion together with UNC5 family of Netrin receptors. (Barallobre MJ. et.al., 2005; Plachez C. and Richards LJ., 2005)

The $d c c$ gene (Deleted in Colorectal Cancer) was originally isolated from the chromosomal region of $18 \mathrm{q}$ in humans, which is commonly deleted in metastatic colorectal cancers (Fearon ER. et.al., 1990). It was considered as a tumor-suppressor gene because of its high $\mathrm{LOH}$ ratios in different cancer types. It has a high level of expression in brain, but also in the basal lamina of several epithelia such as in the gastrointestinal tract, skin, lung, bladder and prostate. The DCC protein was detected in many specific axonal populations, like in lateral olfactory tract, in internal capsule, in corpus callosum, in anterior commissure, in the fimbria/fornix complex, in the fasciculus retroflexus, and in the estria medularis. (Barallobre MJ. et.al., 2005) Loss-offunction phenotype for dcc is quite similar to netrin-1 knockout mice, and lacks the main commissures of the CNS (Fazeli A. et.al., 1997). Alterations in commissural fibers in the spinal cord, and absence of corpus callosum, hippocampal commissure, pontine nuclei, and the appearance of a new ectopic commissure in the interface between hindbrain and midbrain are the main observations in these dcc-deficient mice. Apart from CNS, dcc also plays important roles in the development of visual and olfactory systems, and peripheral nervous system.

Netrin-1 binds to DCC directly from its $5^{\text {th }}$ Fibronection type III repeat, and inducing its multimerization through the association of their cytoplasmic domains, which results in chemoattractive response (Geisbrecht BV. et.al., 2003). DCC is also shown to induce apoptosis conditionally, which puts it in the group of 'dependence receptors'. (Llambi F. et.al., 2001)

Neogenin is a very similar protein to DCC in protein structure. Expression of neogenin is detected mainly in mesodermic tissues of the embryos, but also in low levels within developing brain structures. There is also the high level of expression of neogenin in the axons of retinal ganglionic cells at the beginning of their extension. The neogenin knockout mice do not show severe defects in the nervous system, but for the proper development of mammary glands. (Gad JM. et.al., 1997; Srinivasan K. et.al., 2003) 
UNC-40, which is the only member of the family in nematodes, was identified by forward genetic screenings of C.elegans that give coordination defects as phenotypes. It is shown that UNC-40 guides both mesodermic and neuronal cells in C.elegans. It was also shown to regulate the axon guidance and cell migration dependent on Netrin ligands. (Chan SS. et.al., 1996; Hedgecock EM. et.al., 1990)

\section{UNC5 family of receptors in development of CNS}

UNC-5 was identified in the same screen as mentioned above for UNC-40. Then homologous proteins were identified in other species, which made the UNC-5 family. It contains the original UNC-5 in nematodes; UNC5A, UNC5B, UNC5C (RCM), and UNC5D in vertebrates. These receptors also have a single-span type of transmembrane domains and bind to Netrins with high affinity. Their extracellular domains contain two Immunoglobulin domains and two thrombospondin domains. Their cytoplasmic domains consist of a ZU-5 domain, a DCC-binding domain, and a Death-domain that is similar to the Death-domains of FAS and TNF receptor family. Netrin binding induces the heterodimerization of their intracellular domain with the intracellular domain of DCC, and this heterodimerization converts the Netrin-dependent chemoattraction into chemorepulsion. (Geisbrecht BV. et.al., 2003; Leonardo ED. et.al., 1997) But it is also shown that in the absence of DCC, UNC-5 receptors can also induce chemorepulsion alone. The current belief is that for chemorepulsion, UNC-5 receptors may need the collaboration of DCC receptors when the ligand is in low concentrations. So possibly UNC-5:DCC heterodimers mediate long-range repulsion, whereas UNC-5 homodimers mediate the short-range repulsion as a response to Netrins (Barallobre MJ. et.al., 2005). Similar to DCC, UNC-5 receptors also belong to the 'Dependence Receptors' group, which means they also induce apoptosis in the absence of the netrin ligands. The exact mechanism by which they induce apoptosis is not known, but there are evidences for the involvement of p53, and NRAGE proteins in this process (Tanikawa C. et.al., 2003; Williams ME. et.al., 2003). Recently, it was shown that the mutations of UNC-5 genes were also associated with several cancer types in humans, including colorectal, breast, ovary, uterus, stomach, lung, and kidney (Mehlen P. and Furne C., 2005). In developing mouse brain, all members have distinct but partially overlapping expressions. Apart from brain, unc5A is expressed in neuroretina and whisker pads; unc5B is expressed in 
several vasculatures, neuroretina, and inner ear structures; unc5C expressed in developing limbs; and unc5D is expressed in developing limbs and mammary glands. (Engelkamp D., 2002)

The first member of the UNC-5 family that was functionally studied in vivo, was UNC5C. The RCM (Rostral Cerebellar Malformation)-mouse had a spontaneous nullmutation in the unc5C gene. Mice homozygous for this mutation have several CNS phenotypes, including cerebellar hypoplasia, granule and Purkinje cell mismigration, defects in corticospinal tract pathfinding, and defects in ventral/ipsilateral trochlear nerve misprojections in spinal cord. (Ackerman SL. et.al., 1997; Finger JH. et.al., 2002)

Knockout mice for unc5B was recently published with a phenotype of very early lethality due to the defects in vascular system development ( $\mathrm{Lu} \mathrm{X}$. et.al., 2004). Because of early (E9.5) lethality, we do not really have understanding of its in vivo function during brain and spinal cord development.

Unc-5D was quite recently identified in a screen aiming to find all possible members of UNC5 family. It is the latest expressed member of this family during embryogenesis. But apart from few expressional data, we do not even know if it really binds to Netrins and regulates a chemorepulsive response. (Engelkamp D., 2002)

Unc-5A is the only member whose expression is restricted only to the CNS, retina, and whisker pads. The functional role of unc5A gene during neocortical development will be the aim of this study.

\section{$\underline{\text { Aim of this study }}$}

The cerebral neocortex can be clearly divided into both anatomically and functionally distinct areas, as mapped first by Korbinian Broadmann and others in the beginning of last century. However, its detailed organization and many crucial questions about its area identity, function and developmental establishment of regionalization and lamination are poorly understood.

An approach for gaining better understanding of these questions would be to identify genes that are specifically expressed in distinct regions of neocortex and characterize their roles in the establishment of the structural and functional domains of the cerebral neocortex. 
With the development of advanced molecular biological techniques, the number of genes that can be screened at a time increased from several dozens to thousands. Some of these high throughput techniques are the DNA Microarray Chip assays and SAGE.

Also with the advancement of molecular genetics, the functional roles of these identified genes are being clarified during development or adult life of mice. The transgenic techniques for creating the knock-out or over-expression / ectopicexpression mouse models for specific genes have advanced our understanding of the developmental mechanisms important for neocortex, as well as other tissue or organ structures of mouse. Although mouse and human are not very close relatives to each other in evolutionary terms, it was amazing to see how mouse models of several developmental disorders of humans could be created with these techniques. In summary, recent advances in the field of mouse transgenic technologies are helping scientists to gain deeper understanding for physiological and also patho-physiological development of the neocortex. However, in spite of all these insight, the molecular genetic bases of neocortical development remain largely unknown.

Therefore, in our group, we have attempted to identify genes, which are potentially important for neocortical regionalization and lamination. The loss and gain of function experiments with these genes are very likely to provide important clues for corticogenesis in the mammals. In this regard, we hypothesized that such genes might be predominantly expressed in an area specific manner and attempted to identify them by comparative global gene expression analyses using Affymetrix DNA microarrays.

The selected cortical areas were hippocampus, and motor, somatosensory, visual, and cingulate cortices at three developmental stages of E14.5, E16.5, and E18.5. These stages were specifically chosen based on the two current models of neocortical regionalization. According to the 'protomap model', intrinsic gene expression within the germinal zone plays an important role in corticogenesis (stage E14.5) and decisions are taken before the thalamocortical projections (TCA) reaches their target neocortical structures meaning that the development is only under the control of morphogendependent identity determination mechanism. In contrast, the 'Protocortex model' states that cortical regionalization depends on the information brought by the ingrowing TCA (stage E16.5). At stage E18.5, the principal neocortical regionalization is already finalized. The specific cortical regions from the above mentioned stages were dissected 
and used for a large-scale comparative gene-expression analysis using DNA microarray chips.

I focused on the data obtained for the E14.5 stage and decided to study the functional role of a novel gene with a regionalized expression at this stage. For this purpose, an EST was selected (which was later named as unc5A) from this data set. Identification of this gene was done both in silico and experimentally, and characterized by loss and ectopic gain of function methods. 


\section{RESULTS}

\section{Determination of predominantly expressed genes in different cortical regions of E14.5 mouse embryos}

The DNA microarray chip analysis of E14.5 mouse cerebral cortex was done previously in our group with the purpose of detection and isolation of region-specific genes. Selected cortical regions for this analysis were: Dorso-frontal cortex (motor cortex), parietal cortex (somatosensory cortex), dorso-caudal cortex (visual cortex) and caudo-medial cortex (hippocampus). Briefly, these selected cortical regions of E14.5 mouse brains were isolated and total RNA samples were extracted for each of them. These RNA samples were used as templates for oligo-dT primed cDNA synthesis. Labeled RNA probes were prepared from these cDNA samples by in vitro transcription with biotinylated nucleotides and hybridized with the microarray DNA chips (from Affymetrix, MGU74). The fluorescence signals coming from hybridizations on each spot of microarrays were scanned and their intensities were quantified. The results for all selected cortical regions were compared with each other in order to calculate the 'fold-change' values.

The data set for the E14.5 stage contained a total of 369 genes with 216 showing predominant expression in one of these regions compared to at least one other region according to the criteria of " max-fold change $>3.0$ " (our unpublished observation).

The number of genes detected as predominantly expressed in hippocampus or somatosensory cortex were much higher than the number of genes for visual or motor cortex at this embryonic stage (Figure 1A). There were 128 genes for the hippocampus, 58 genes for the somatosensory cortex, 15 genes for the motor cortex and 15 genes for the visual cortex. Among all the tissues analyzed, hippocampus belongs to the "old cortex", whereas all the others are parts of the " new cortex" (neocortex). Because of being evolutionarily distinct, one can expect that the set of genes responsible for its development might be also distinct. It is also known that the development of hippocampus and somatosensory cortex at the embryonic stage of E14.5 is much more advanced than motor cortex or visual cortex. 
According to the annotations of the microarray chips, we found that 54 of these 216 genes were already identified genes. The bioinformatical analysis of the remaining 162 genes, which were considered as ESTs, was done. By aligning the sequences of microarray probes for these genes with the Genbank database (NCBI), I identified 26 of them as being genes already identified in mouse; 36 of them as being genes identified only in some other species, like human or rat; and 100 of them as being unknown genes. All these known genes have been categorized according to the known functions of proteins that they encode for (Figure 1B). Majority of the known genes fall into the groups of transcription factors and signaling molecules. There were also several "Putative genes", which are the bioinformatically predicted genes from human genome sequences.

I then did the in silico gene-prediction studies for the remaining 100 unknown genes. The genomic databases and gene-prediction programs from Celera, NCBI, and Ensembl were used. Out of these 100 genes, 23 of them did not contain any openreading frame and 12 of them did not match with any previously isolated EST sequences deposited in the Genbank Database (NCBI). However, the putative partial or complete protein sequences for the other 65 of them were determined. Using the Blast programs of Celera, NCBI, Ensembl, and ProScan, I searched their possible protein motifs and homologies to known proteins. Functions of these genes were predicted based on these bioinformatical findings: 12 of them for a function in the metabolic pathways of mitochondria; 17 of them for the cytoplasmic metabolism; and 7 of them for being extracellular matrix proteins. 14 of them do not have any predicted protein motifs, but only similarities to some known proteins with unknown function. The remaining 15 predicted genes had homologies to many previously identified "Putative genes" from human genome. Their predicted functions include a set of transcriptional regulators, translational regulators, axon guidance molecules and WNT-signaling pathway molecules. 


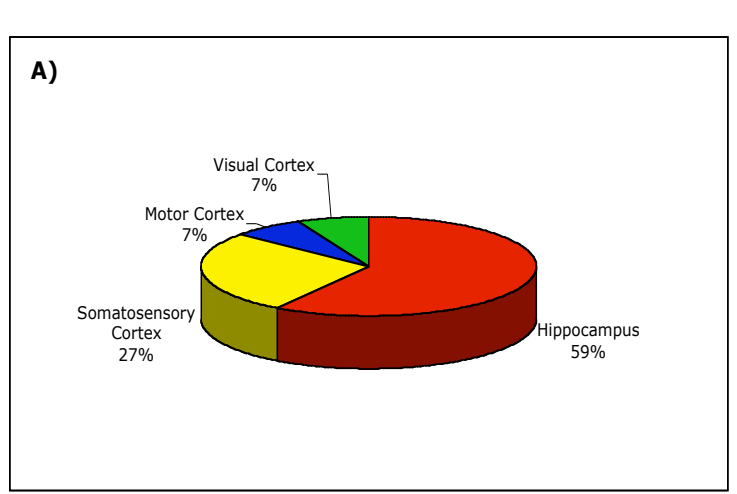

B)

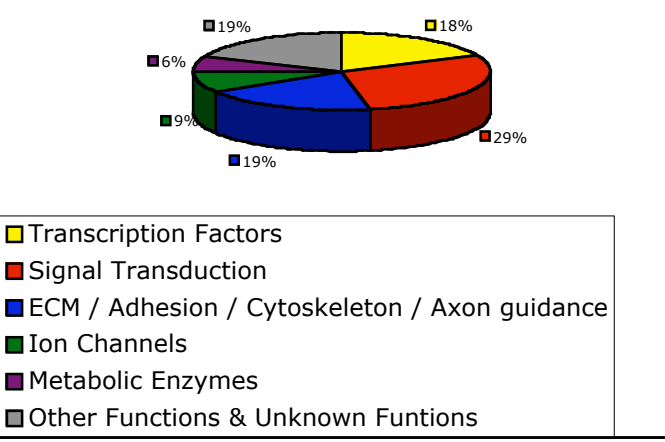

Figure 1: Analysis of microarray DNA chip data for the determination of predominantly expressed genes in different cortical regions of E14.5 mouse embryos.

A: Distribution of the predominant expression area of the 216 identified genes to various cortical regions. B: Categorization of all the known genes in the microarray data set according to the functions of proteins they encode for. 


\section{In situ hybridization analysis of selected genes from the} microarray data

21 of the 216 genes were selected for further analysis based on the known or predicted functions of the proteins encoded by these genes. The main principle for the selection was their being possibly important for the developmental processes, like being transcriptional or translational regulators, signaling pathway molecules, or axon guidance molecules. The corresponding EST clones were obtained from IMAGE consortium. In situ hybridization (ISH) analysis was done for each of them in order to confirm the chip data and also to see the expression patterns of these genes in the other brain regions that were not included in the chip analysis. As a starting point, the ISH was done on E14.5 brain sections in order to verify the chip data. For additional selected genes, the ISH was also done on E18.5 brain sections in order to see their expression patterns for developmentally older embryonic brains. Here, I will mention only three of them.

AU-18, which has been identified later as $u n c 5 A$, showed predominant expression in the visual cortex. According to the microarray data, the fold of expression in visual cortex was 4.9, 3.2, and 3.1 times higher than in somatosensory cortex, motor cortex, and hippocampus, respectively. ISH results for this gene on E14.5 mouse brain sections showed strong expression on the cortical plate of visual cortex, basolateral cortex, and pyriform cortex (Figure 2A-E). The expression pattern of unc5A is also respecting the boundaries of "Internal Capsula" within the telencephalon (Figure 2C-E). Strong expression on epithalamus, and "supraoptic area", and weaker expression in "dorsomedial hypothalamic nucleus" and "central nucleus of amygdala" was also observed (Figure 2C-E). The results of ISH on E18.5 mouse brain sections showed even more striking anterior-low and posterior-high gradient of expression. The expression level in visual cortex was much higher than in motor cortex or somatosensory cortex (Figure 3A-F). The expression in pyriform cortex and "central nucleus of amygdala" remained visible in this later stage of development (Figure 3A and 3D). The expression observed in " Rostral migratory Stream (RMS)" was also strongly visible (Figure 3A and 3B). Within hippocampus, CAIII and dentate gyrus regions were strongly positive whereas CAI and CAII were not (Figure 3D). Weaker expressions were observed in 
septum and ventromedial hypothalamic nuclei. Many important brain structures that are important for the visual system, like "medial preoptic nucleus", and "collicius superior" were showing remarkably specific expression (Figure 3A and $3 \mathrm{E}$ ). The expression of unc $5 \mathrm{~A}$ in epithalamus and hypothalamus was also observed in this embryonic stage. At E18.5 stage, it was also remarkable that there was a strong expression in dorsal thalamus, whereas no expression in ventral thalamus (Figure 3C).

AU-16 (identified later as $k l h d c 8 A$ ) showed, according to our microarray data, predominant expression in hippocampus with fold-changes of 2.2, 2.4, and 3.1 times higher than in somatosensory cortex, motor cortex and visual cortex, respectively. ISH analysis for this gene on E14.5 mouse brain sections showed weak expression in the hippocampal anlage, and no detectable expression in any other cortical regions (Figure $2 \mathrm{~F}-\mathrm{H})$. The expression was also detected in some specific nuclei of hypothalamus, "medial longitudinal fasciculus", and pons (Figure 2I-J).

According to our microarray data, AU-4 (identified later as satB2) showed the predominant expression in somatosensory cortex with the fold changes of: 2.3, 2.0 and 16.4 times higher than in visual cortex, motor cortex and hippocampus, respectively. This data suggests that AU-4 is expressed only in the 'neocortex' region of the cerebral cortex. ISH analysis for this gene on E14.5 mouse brain sections showed very strong and restricted expression in the basolateral cortex and somatosensory cortex only (Figure 2K-M). Apart from the cerebral cortex, the weak expression in dorsomedial hypothalamic nucleus was also observed (Figure 2M). We detected high expression levels of this gene in the cortical plate of cingulate cortex, parietal (somatosensory) cortex, basolateral cortex, and visual cortex by ISH analysis on E18.5 mouse brain sections (Figure 3G-L). There was no expression in hippocampus, or in diencephalon. This data suggests that AU-4 is expressed solely in neocortical structures within the developing mouse brain.

The ISH data on E14.5 mouse brain sections confirmed by and large the microarray data. Based on the expression patterns of the 21 selected genes, 3 were chosen for further analysis. Their expression patterns are shown in figures 2 and 3. 


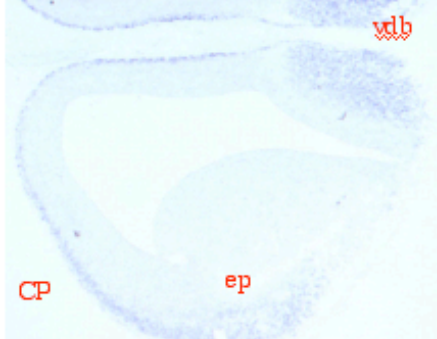

D

Epth

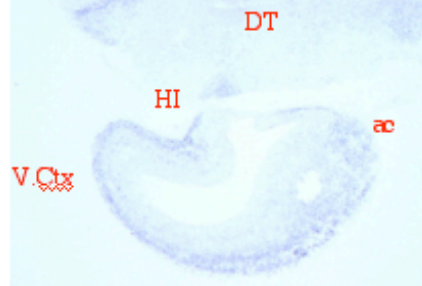

F

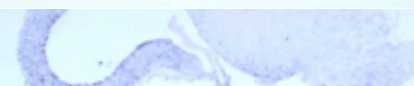

I

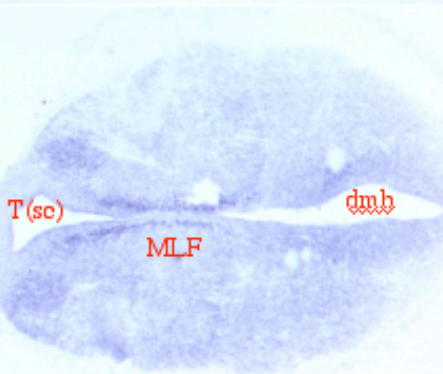

K

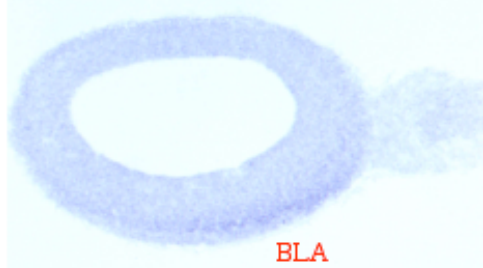

B

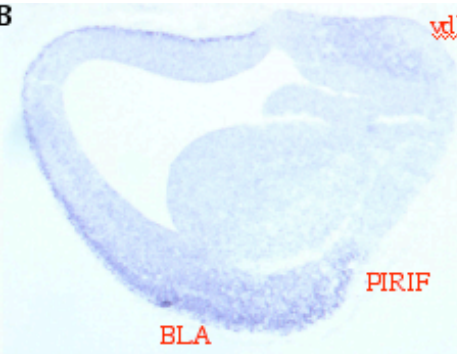

$\mathrm{db}$

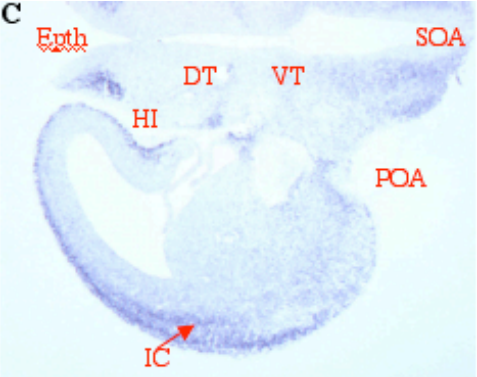

G

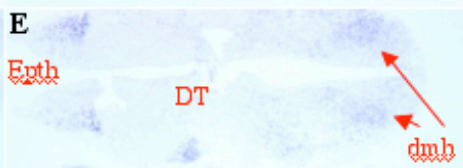

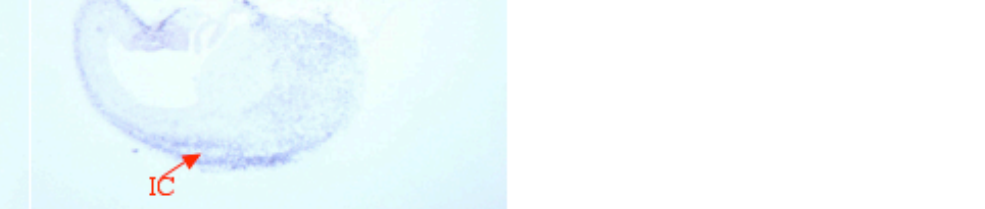

H

Hnth

HI

J

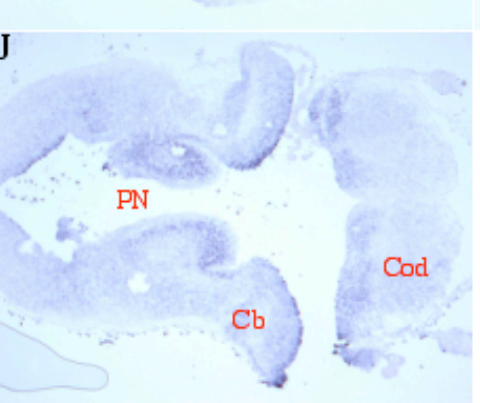

L

M

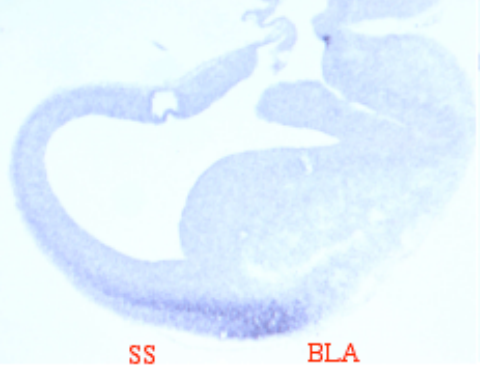

Figure 2. In situ hybridization analysis on coronal sections of E14.5 mouse embryonic brains for some of the selected genes from microarray data.

A-E: AU-18 (unc5A) showed the cortical expression in the cortical plate of visual cortex, basolateral cortex, and pyriform cortex. F-J: AU-16 (klhdc8A) showed the cortical expression in hippocampal anlage. K-M: AU-4 (satB2) showed the cortical expression in basolateral cortex and somatosensory cortex. (ac: central nucleus of amygdala, BLA: basolateral cortex, $\mathbf{C b}$ : cerebellum, Cod: dorsal cochlear nucleus, CP: cortical plate, dmh: dorsomedial hypothalamic nucleus, DT: dorsal thalamus, ep: entopeduncular nucleus, Epth: epithalamus, HI: hippocampus, Hpth: hypothalamus, IC: internal capsula, MLF: medial longitudinal fasciculus, PIRIF: pyriform cortex, PN: pons, POA: preoptic area, SOA: supraoptic area, SS: somatosensory cortex, $\mathbf{T}$ : tectum, Vdb: vertical nucleus of diagonal band of Broca, VT: ventral thalamus) 


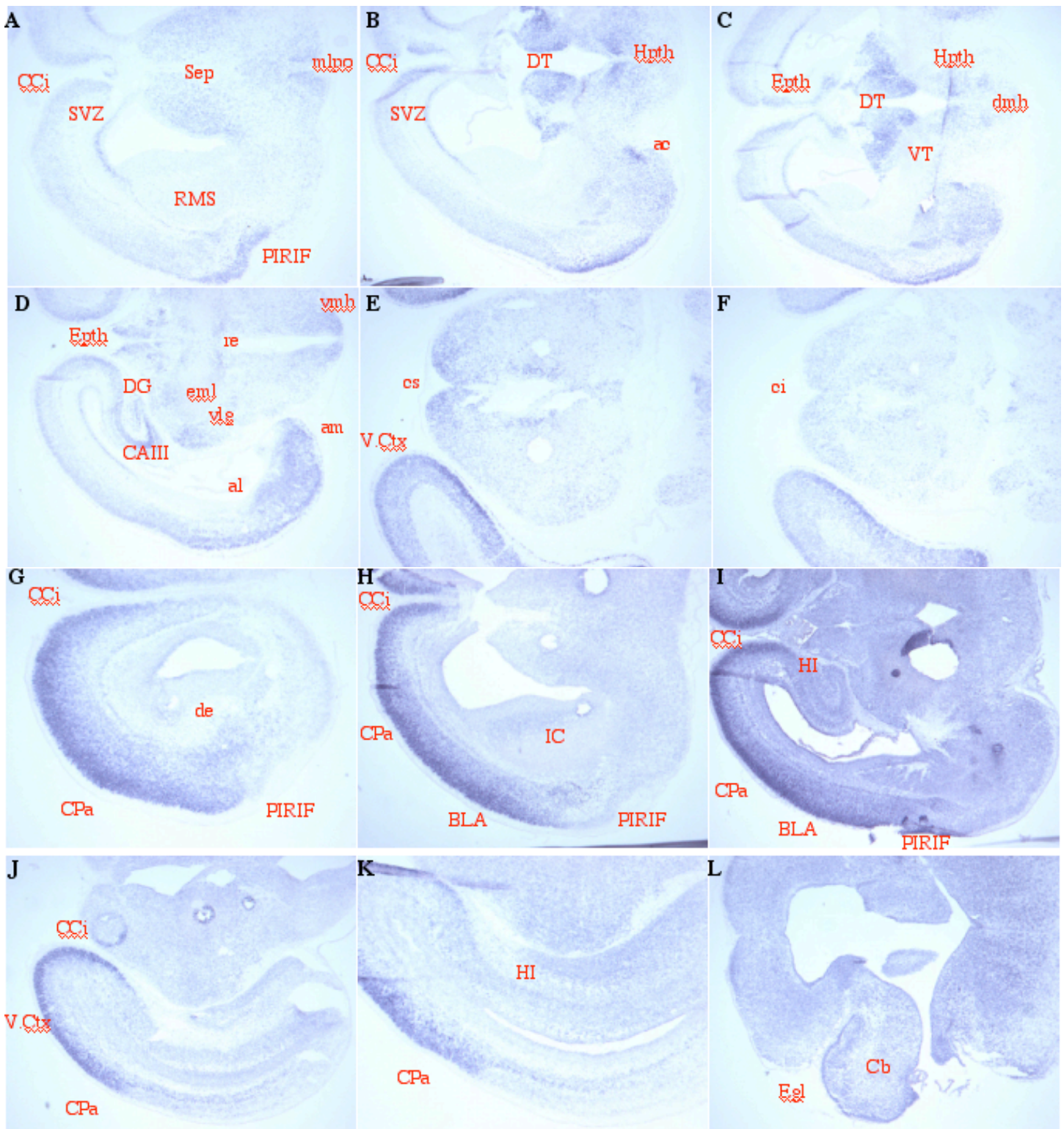

Figure 3. In situ hybridization analysis on coronal sections of E18.5 mouse embryonic brains for selected genes.

A-F: AU-18 (unc5A) showed anterior-low and posterior-high gradient of expression within cerebral cortex. G-L: AU-4 (satB2) showed high expression levels in the cortical plate of cingulate cortex, parietal (somatosensory) cortex, basolateral cortex, and visual cortex.

(ac: central nucleus of amygdala, al: lateral amygdaloid nucleus, am: medial amygdaloid nucleus, BLA: basolatareal cortex , $\mathbf{C b}$ : cerebellum, CCi: cingulate cortex, ci: coliicius inferior, $\mathbf{C P a}$ : Parietal cortex, cs: collicius superior, de: doeral endopyriform nucleus, DG: dentate gyrus, $\mathbf{d m h}$ : dorsomedial hypothalamic nucleus, DT: dorsal thalamus, Egl: external germinative layer, eml: external medullary lamina, Epth: epithalamus, HI: hippocampus, Hpth: hypothalamus, IC: internal capsula, mlpo: medial preoptic nucleus, PIRIF: pyriform cortex, re: reuniens nucleus, RMS: rostral migratory stream, Sep:. septum, SVZ: subventricular zone, vlg: ventral lateral geniculate body, vmh: ventromedial hypothalamic nucleus, VT: ventral thalamus) 


\section{$\underline{\text { In silico identification of gene and protein structures }}$}

Full cDNA sequences for these three selected genes were deduced bioinformatically, by aligning all the matching EST sequences from Genbank database in several steps. Afterwards, these putative cDNA sequences were aligned with the mouse genomic DNA sequences from Celera and Ensembl databases in order to predict the exon-intron structures for each of them.

AU-18 (unc5A) is located on chromosome 13 of the mouse genome, and contains 16 exons spanning approximately $57 \mathrm{~kb}$ region in genome. All 16 exons contribute to the open-reading frame. First exon contains a short $5^{\prime} \mathrm{UTR}$, and the $16^{\text {th }}$ exon, which is the longest exon of all, contains quite a long $3^{\prime}$ UTR sequences. It encodes a cDNA of 3992 bps, and a protein of 898 amino acids. The predicted motifs of the UNC5A protein contain a "signal peptide" on the $\mathrm{N}$ terminus of the protein, two IG (Immunoglobulin) domains, and two TSP (Thrombospondin) domains as its extracellular part; a singlespan type of transmembrane domain; and in the intracellular part the ZU-5 domain, the DCC-binding domain and the DEATH domain (Figure 4A).

Based on the predicted protein motifs and homology searches to known proteins, this gene was determined as an axon guidance receptor for Netrin ligands. It has high homology to $\mathrm{rcm}$ (rostral cerebellar malformation) gene of mouse, and unc5 gene of C.elegans. Later on, it was identified and published with the name of unc $5 \mathrm{H} 1$ or unc $5 \mathrm{~A}$ (Engelkamp D., 2002).

AU-16 (khldc8A) is located on chromosome 1 of the mouse genome. It contains 5 exons within a $7.5 \mathrm{~kb}$ genomic region. All exons contribute to the open-reading frame. There are quite short 5'UTR sequences in the first exon and $3^{\prime}$ UTR sequences in the last exon. This gene encodes a 2217 bps cDNA and produces a protein of 350 amino acids. The protein contains 4 copies of KELCH motifs only (Figure 4B). These KELCH motifs generally function in protein-protein interactions. Homology searches for this gene detected several similar proteins, which all contain KELCH motifs. Later on, it was identified as khldc8A (Kelch-Domain-Containing protein 8A). KELCH-proteins can function in many different pathways. Therefore, it is hard to predict a specific function for khldc8A. Consequently, this gene was not selected for further functional studies. 
AU-4 (satB2) is also located on chromosome 1 of the mouse genome. It contains 16 exons within a $176 \mathrm{~kb}$ of genomic region. The exons 5 to 10 contribute to the openreading frame, and there are quite long $5^{\prime}$ and $3{ }^{\prime}$ UTR sequences coming from the first four exons and last six exons, respectively. It encodes a cDNA of approximately 4100 bases and a protein of 524 amino acids. Predicted protein motifs are two CUT-domains and one homeodomain (Figure 4C).

This gene is very similar to the known genes of CUT transcription factors subfamily. It is the first mammalian member that contains two CUT domains since all the mammalian members identified up to now contain either one or three CUT domains. Both the CUT domains and the homeodomain have the ability to bind to the DNA. These features make this gene a very important candidate to investigate its function in cortical development. However, this gene was also not selected for further functional studies because its both loss-of and gain-of function mutants were created already but not published yet (personal communication with Tarabykin V.).

After the determination of the expression patterns and predicted gene structures with protein motifs for these 3 selected genes, I decided to analyze the functional role of AU-18 (unc5A) in neocortical development and regionalization via creating the loss-of and gain-of function mutant animals.

Since unc5A encodes for a Netrin-dependent chemorepulsive receptor, we hypothesized that it may play important functional roles in the structural and functional development of the neocortex. It is already known that besides the regulation of the axonal pathfinding, netrin dependent axon guidance systems are also important for the regulation of cellular migrations. Its embryonic expression in several forebrain and midbrain regions important for the visual system (like visual cortex, internal capsule, medial preoptic nucleus and collicius superior) and for the olfactory system (like pyriform cortex and rostral migratory system) have been detected and shown above. It is also expressed in the embryonic neuroretina and whisker pads (Engelkamp D., 2002). Based on these expressional patterns of $u n c 5 A$, we finally postulated that it may have crucial roles in the development of not only the visual and olfactory, but also the whisker-related somatosensory systems. 


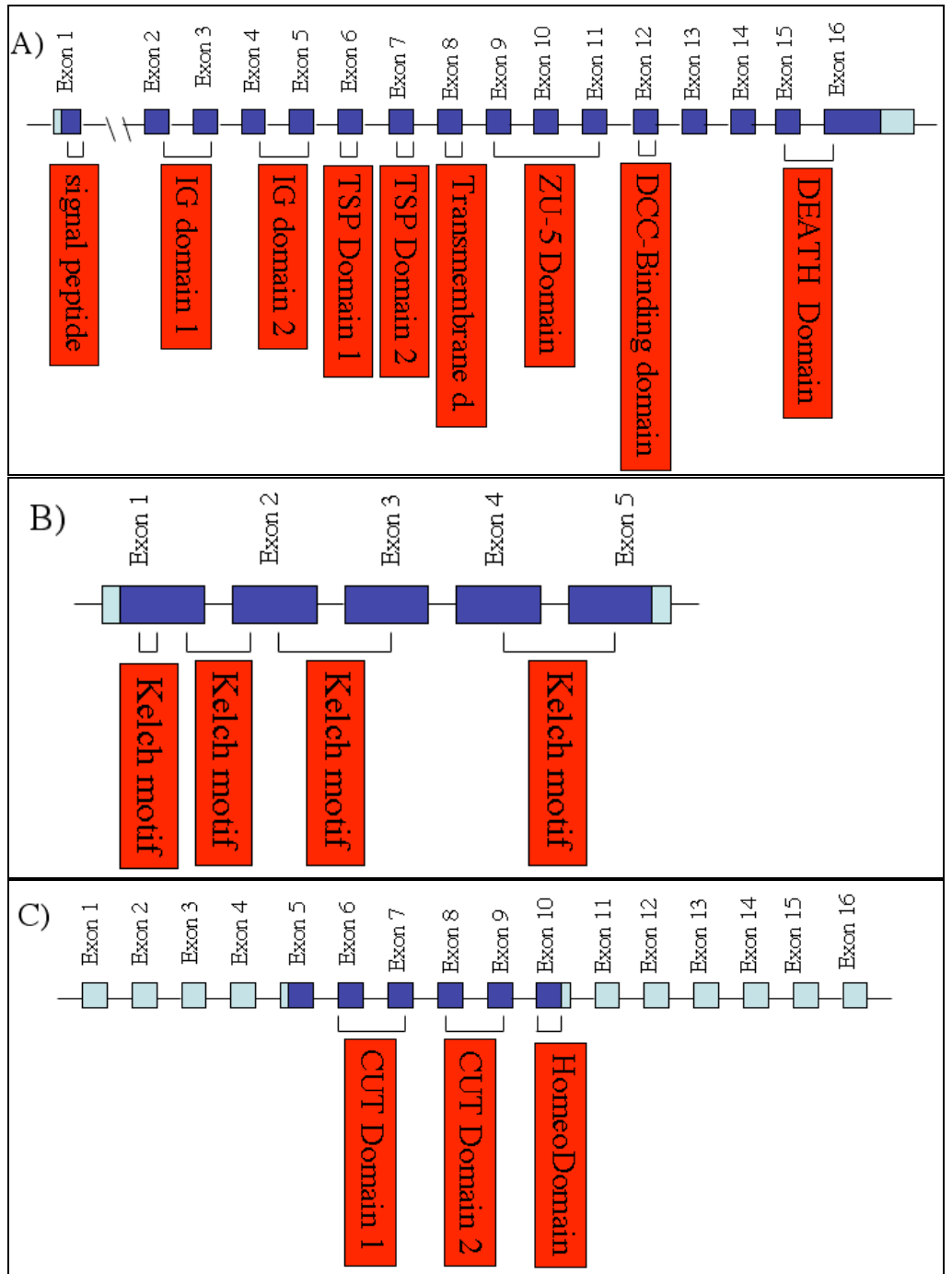

Figure 4. In silico identification of gene and protein structures.

A: AU-18 (unc5A). B: AU-16 (khldc8A). C: AU-4 (satB2)

All coding exons are shown as dark blue rectangular boxes and non-coding exons are in pale blue. The flanking genomic regions and introns are shown as horizontal lines. Predicted protein motifs for each are shown in red-colored rectangular boxes under the exons, which encode for them. 


\section{Identification of gene-structure of unc5A by RACE and RT-PCR}

\section{$\underline{\text { Analysis }}$}

To experimentally confirm the in silico-predicted gene structure for unc5A, RACE and RT-PCR methods were used. Total RNA isolated from E18.5 mouse embryonic brains was used for oligo-dT primed cDNA synthesis. The $3^{\prime}$ end of the cDNA was determined by $3-$ RACE analysis by using two nested forward primers designed from the $14^{\text {th }}$ and $15^{\text {th }}$ exons, respectively. The DNA bands with expected sizes were obtained after 2 steps of nested-PCR amplification. These PCR fragments were cloned and sequenced. The obtained sequences confirmed the 3 end sequences of the in silico predicted gene.

In order to determine the $5^{\prime}$ end of the gene with the transcriptional start site, $5^{\prime}-$ CAP-RACE was done from the same E18.5 mouse brain total RNA. Reverse nested primers were designed from the $2^{\text {nd }}$ and $3^{\text {rd }}$ exons. By using the GENE-RACER kit (Invitrogen), the intact total RNA, which contains the 5'-CAP structures, were ligated to oligo linkers. The reverse-transcription was done with a gene-specific reverse primer from the $4^{\text {th }}$ exon. The PCR fragments obtained after 2 steps of nested-PCR reactions were cloned and sequenced. The obtained sequences confirmed the predicted $5^{\prime}$ end of the $u n c 5 A$ cDNA.

Many of the EST sequences in Genbank database were suggestive of a possible spliced variant form for $u n c 5 A$, in which the $6^{\text {th }}$ exon has been spliced out. PCR primerpairs from the $4^{\text {th }}$ and $7^{\text {th }}$ exons were designed to identify such a putative spliced variant ( $\Delta$ exon6). As seen in Figure 5, the highly expressed form of unc5A during embryogenesis is actually this " $\Delta$ exon6" spliced variant. In order to discover other potential spliced variants, several PCR primer pairs were designed encompassing other exons. However, no additional splice variants were detected.

Soon after I finished these gene-identification experiments, Engelkamp D. (2002) published the structure of mouse $u n c 5 A$ gene and its only spliced variant ( $\Delta$ exon6). The published results matched completely to our data.

Expression kinetics of unc5A during embryogenesis was analyzed by RT-PCR using total RNAs isolated from embryonic stages E8.5 and E18.5 (Figure 5A). Unc5A expression was detected at all embryonic stages tested and the " $\Delta$ exon6" turned out to 
be the major transcript. During the stages between E13.5 and E15.5, the expression level of the full-length transcript reached its peak, but then declined to a lower level. The expression levels of " $\Delta$ exon6" spliced variant remained unchanged throughout all stages tested.

Since the increase of body size is much higher than the head size during the development after E13.5, I decided to check the expressional dynamics of unc5 A separately for body and head samples for the embryos older than E13.5. For this purpose, several embryos of the stages between E14.5 and E18.5 were cut into "head" and "body" on the lower parts of the neck above the shoulders. After total-RNA isolation and cDNA synthesis, the expression of $u n c 5 A$ was analyzed with the same PCR reactions (Figure 5B). Among the "head" samples, the expression of both variants of $u n c 5 A$ seems to remain unchanged between different embryonic stages. For the "body" samples, we observed a decrease in the expression of both variants starting from the E17.5 stage. 

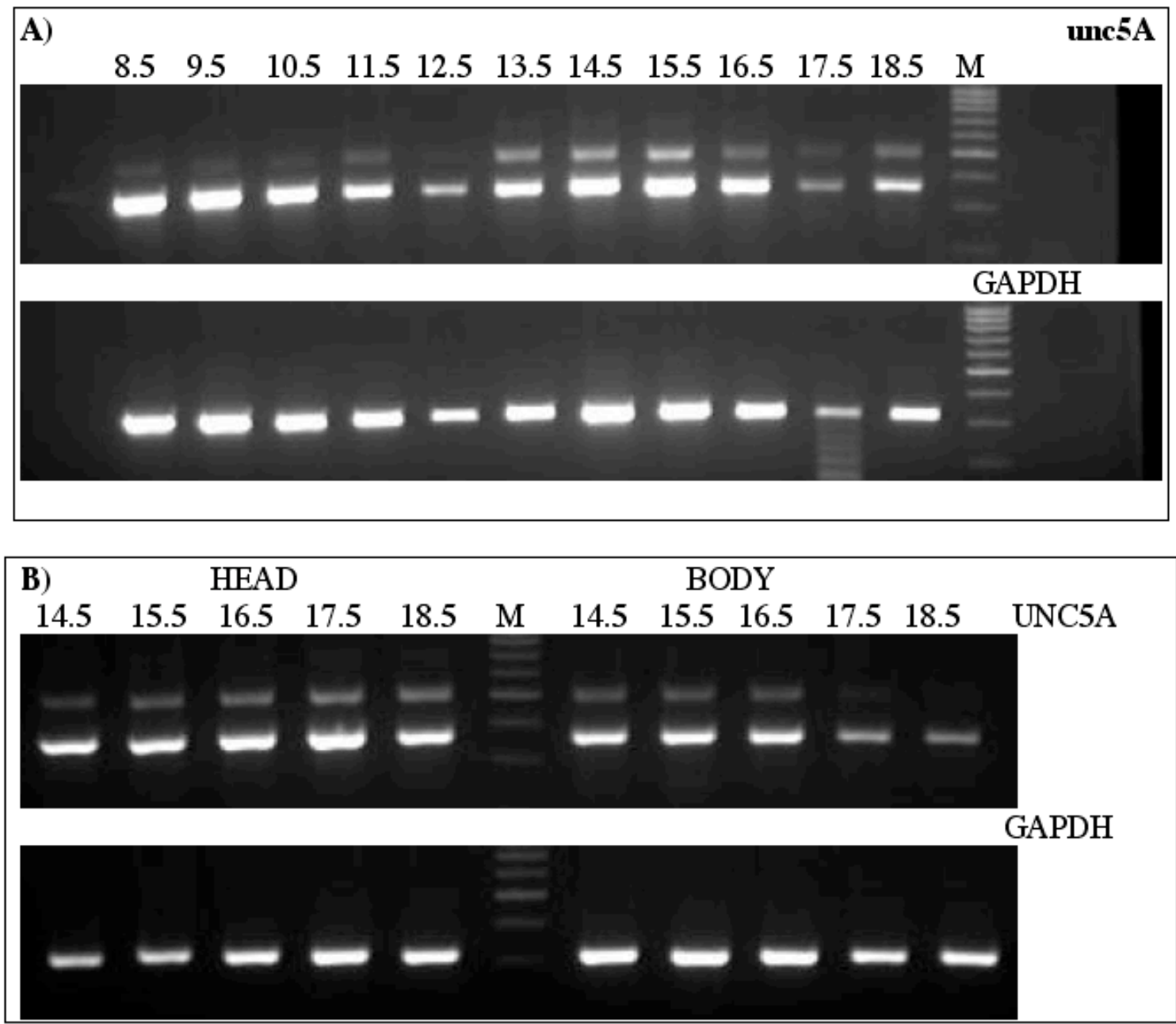

GAPDH

Figure 5. Expressional kinetics for both variants of unc5A during embryogenesis.

A: Analysis for whole embryos between stages E8.5 and E18.5 B: Analysis for "head" and "body" samples separately, between stages E14.5 and E18.5

Upper rows on each panel are for unc5A specific-PCR, and lower rows for GAPDH specific-PCR, which is used in order to normalize the differences coming from the density of cDNA samples. The upper bands on the unc5A-PCR results correspond to the full-length form (530 bps), whereas the lower bands for the " $\Delta$ exon6" variant (365 bps). $\mathrm{M}$ is the 100-bp ladder. 


\section{Generation of knockout constructs and ES-cell screens}

In order to create the loss-of-function mutants for unc $5 A$, a conventional knockout construct was generated. Both $5^{\prime}$ and $3^{\prime}$ homology arms were produced by PCR from isolated ES-cell genomic DNA. 5’ homology arm was 4981 bps -long and contained sequences between $2^{\text {nd }}$ intron and $9^{\text {th }}$ exon, whereas the $3^{\prime}$ homology arm was 4991 bpslong and contained the flanking genomic sequences downstream of the last exon (Figure 6A). This construct would delete unc $5 A$ gene sequences located between the exon 9 and the end of the gene encoding the intracellular part of the unc5A protein.

"IRES-TauLacZ-pA" cassette was inserted at the 3' end of 5'homology arm to achieve a knock-in of 'TauLacZ' into the unc5A locus. TauLacZ fusion protein was selected as a reporter, because it would give us an opportunity to trace the axonal tracts via LacZ staining. It is known that TauLacZ fusion protein is generally transported into the cytoskeleton of the long axonal structures due to the 'Tau' part of the fusion protein. This cassette contained its own polyA signal sequence to stop the transcription and IRES (Internal Ribosome Entry Site) sequence to ensure that it will be translated independent of the open-reading frame of unc $5 \mathrm{~A}$ gene. Since this cassette would be replacing the $u n c 5 A$ sequence downstream of the $9^{\text {th }}$ exon where the $5^{\prime}$ homology ends, the transcriptional control of TauLacZ gene will be under the control of transcriptional regulators of unc $5 A$ gene, and will mimic its expression. A floxed "pGK-NeomycinpA" cassette was placed between "IRES-TauLacZ-pA" cassette and 3 'homology arm in order to use it for positive selection of recombined ES-clones afterwards. Within this cassette, neomycin gene has its own strong pGK promoter and polyA signal sequence. The LoxP sites which are on both ends of the cassette in the same orientation gives us the possibility of removing the whole neomycin cassette after the selection of ES cells and production of chimaeras. For the negative selection, a thymidine kinase (TK) cassette with its own promoter and polyA sequences was inserted downstream of 3 'homology arm. (Figure 6A)

Since the homology arms were produced by PCR, both homology arms of the construct were extensively sequenced in multiple overlapping regions in order to see that PCR did not create any mutations during the amplifications. The obtained DNA sequences were compared with the genomic sequences from CELERA, Ensembl and NCBI databases. We had more than $99.9 \%$ similarity to the database genomic 
sequences. The detected few mutations were all in the intronic sequences within 5 'homology arm. However, the minor sequence differences could also be attributed to the different mouse strains.

The linearized construct was electroporated into (MPI-II) ES-cells. Positive selection for neomycin and negative selection for TK were done and the 255 surviving clones were picked and grown separately. Genomic DNAs were prepared, digested with EcoRI, electrophoresed into agarose gels and transferred to nylon membranes according to the Southern blotting protocol. The membranes were hybridized separately with corresponding 5' and 3' external probes. Expected band sizes for the wild-type (WT) allele with both probes was $21.0 \mathrm{~kb}$; whereas for mutant alleles with correct homologous recombination, the expected band sizes were $6.3 \mathrm{~kb}$ with the $5^{\prime}$ probe and $7.6 \mathrm{~kb}$ with the 3' probe. After screening all $255 \mathrm{ES}$-cell clones, no positive clone was obtained. (Figure 6A)

As the next attempt to create a loss-of-function mutant, I decided to create a “Conditional Knock-Out" construct (Figure 6B). By using 2 different overlapping mouse genomic $\lambda$-phage clones, I prepared a 3740 bps-long $5^{\prime}$-homology arm, an 8424 bps-long internal homology arm and 4273 bps-long 3'-homology arm. The 5'homology arm contained sequences between $2^{\text {nd }}$ intron and $7^{\text {th }}$ intron. Internal homology arm contained the genomic sequences starting from exactly where the $5^{\prime}$-homology arm was ending and continued till the region downstream of the last exon. A $4.2 \mathrm{~kb}$ genomic region starting downstream from the point where the internal homology arm ended, represented the 3'-homology arm. For the conditional knockout strategy, 2 LoxP sites were placed in the same orientation in the $7^{\text {th }}$ intron and in the downstream sequences after the end of the last exon, to encompass the entire internal homology arm. This would target all exons that are encoding the whole intracellular and transmembrane domains of the unc5A protein (Figure 6B). A neomycin cassette with a strong PGK promoter and polyA signal sequence together with flanking FRT sequences on both ends of the cassette (in same orientation) was placed in between the $5^{\prime}$-homology and the internal homology arms. For the negative selection of ES-cells, TK cassettes with its own promoter and polyA signal sequences were placed at both upstream of 5 homology and downstream of $3^{\prime}$ homology arms (Figure 6B).

The linearized construct was electroporated and 107 clones were obtained after positive and negative selection as mentioned earlier. Genomic DNAs from all clones 
were checked for correct integrations at both $5^{\prime}$ - and $3^{\prime}$-end with external probes. Expected band sizes for the wild-type (WT) allele with both probes were $21.0 \mathrm{~kb}$; whereas for mutant alleles with desired homologous recombinations, the expected band sizes were $5.0 \mathrm{~kb}$ with 5'probe and $7.6 \mathrm{~kb}$ with 3'probe (Figure 6B).

From the 107 ES-cell clones tested, no positive clone for correct integration at 5' or 3' end could be obtained (Figure 6B).

Since the number of clones obtained after positive and negative selections were really low in this electroporation, in the next attempt, we decided to remove one of the TK cassettes in this construct in order to weaken the selective pressures. With this purpose, a third construct was prepared in a similar way as the previous construct, but only one TK cassette was added downstream of 3'-homology arm (Figure 6C). After linearizing this construct, it was electroporated into the ES-cells. We had 159 ES-cell clones after both the negative and positive selections. Isolated genomic DNA samples from each of these 159 clones were checked with the same Southern-Blot Screening method as mentioned for the previous conditional knockout construct. As a result, we had no ES-cell clone that is positive for both 5' and 3' screens (Figure 6C). However, this time we had 2 clones, which were positive only for the $5^{\prime}$ screen but negative for the 3' screen. Obviously, these 2 ES-cell clones had undergone homologous recombination between the 5' homology and the internal homology arms instead of between $5^{\prime}$ and 3' homology arms. The recombined locus therefore maintained the neomycin cassette and only one LoxP site. But the presence of only one LoxP site would not allow us to create the loss-of-function mutants. For this reason, these ES-cell clones were also discarded.

On the other hand, the successful homologous recombination event gave us the hope that this genomic locus can be accessed by keeping the homology arms long enough. However, the percentage of clones with homologous recombination in this locus was still less than $0.5 \%$. Since it is always harder to get the correct homologous recombination with a conditional knockout construct (as compared to a conventional knockout construct), we next decided to create a conventional construct with a larger homology.

In this construct, the $5.9 \mathrm{~kb}$-long $5^{\prime}$ homology arm contained the genomic sequences located between the $1^{\text {st }}$ intron and $4^{\text {th }}$ exon and the $7.1 \mathrm{~kb}-$ long $3^{\prime}$ homology arm contained the genomic region located downstream from the last exon (Figure 6D). 
An "IRES-TauLacZ-pA" cassette was placed just after the 5' homology arm, which would replace the deleted region of $4^{\text {th }}$ exon and other downstream exons after homologous recombination. For the positive selection of ES-cell clones after electroporation, we placed a neomycin Cassette with a strong PGK promoter and polyA signal sequence together with FRT sequences on both end of the cassette (in same orientation). A TK cassette with its own promoter and polyA signal sequence was placed on downstream of 3'homology arm for the negative selection of ES-cell clones. (Figure 6D)

After the linearization of this construct, it was electroporated into the ES-cells. After the positive and negative selections, we had 310 ES-cell clones. The genomic DNA samples for each clone was prepared and analyzed by Southern Blot Screening methods with both $5^{\prime}$-internal and $3^{\prime}$ - external probes. Two positive ES clones were detected: ES\#23 and ES\#264 (Figure 6E). After intensive analysis of these clones with different restriction enzymes and hybridization probes, we realized that the clone ES\#23 was correctly integrated, but clones ES\#264 had two copies of the targeting construct, in which one was integrated into the correct place via homologous recombination, whereas the other was integrated into another genomic locus via non-homologous recombination.

The same knockout construct (Figure 6D) was electroporated one more time into the ES-cells in order to get some more positive clones. After the same set of selections, we got 210 ES-cell clones. Among these clones, we identified 1 ES-cell clone (ES\#110) as positive for both $5^{\prime}$ and $3^{\prime}$ Southern Blot screenings (Figure 6E). 

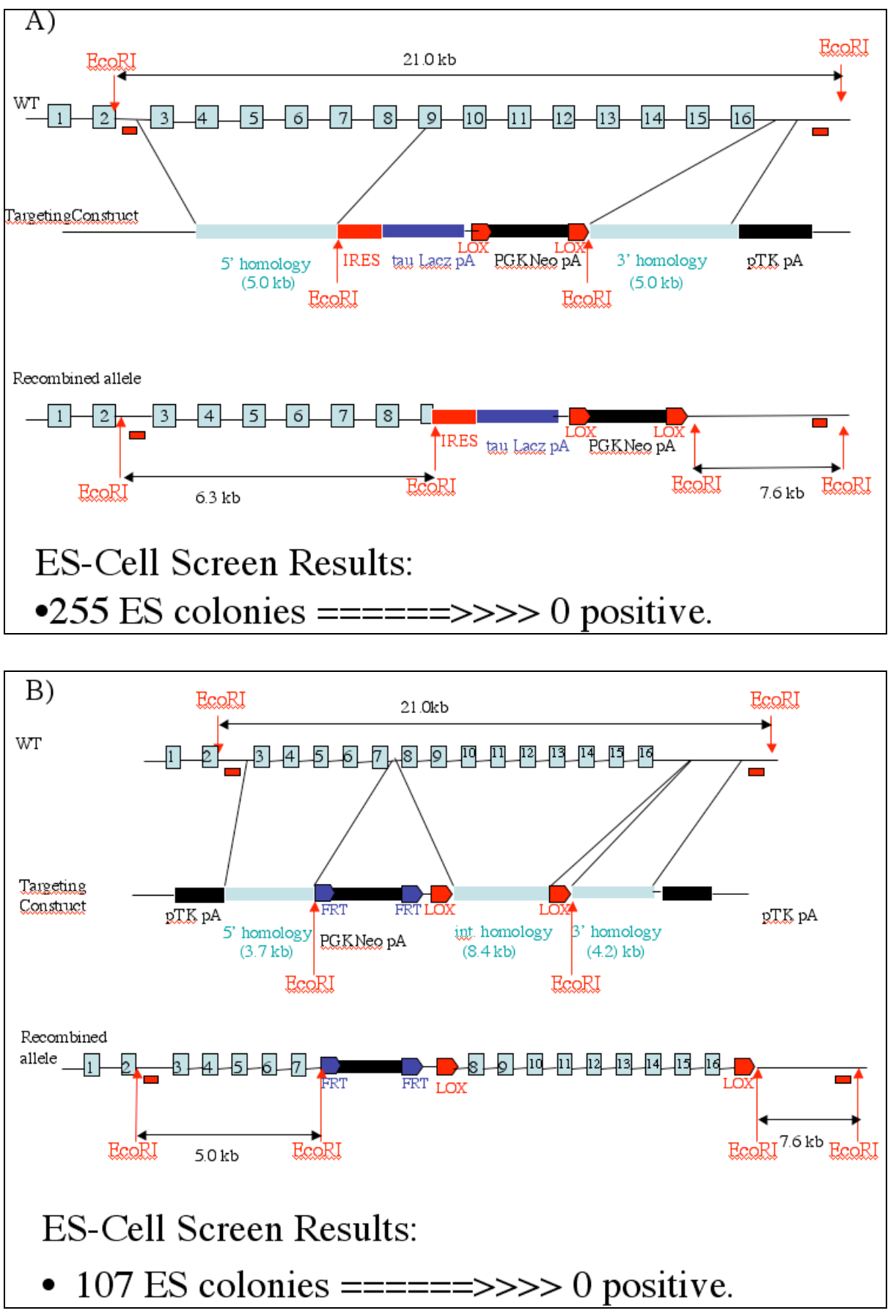


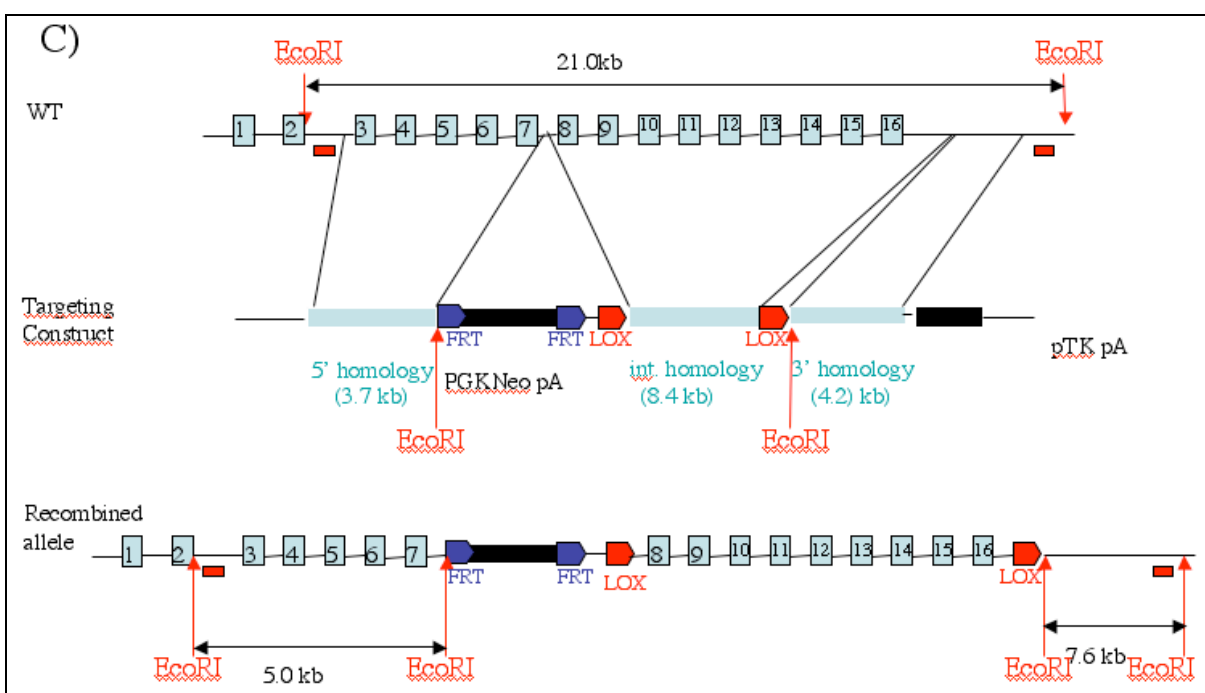

ES-Cell Screen Results:

- 161 ES colonies ======>>>> 0 positive.

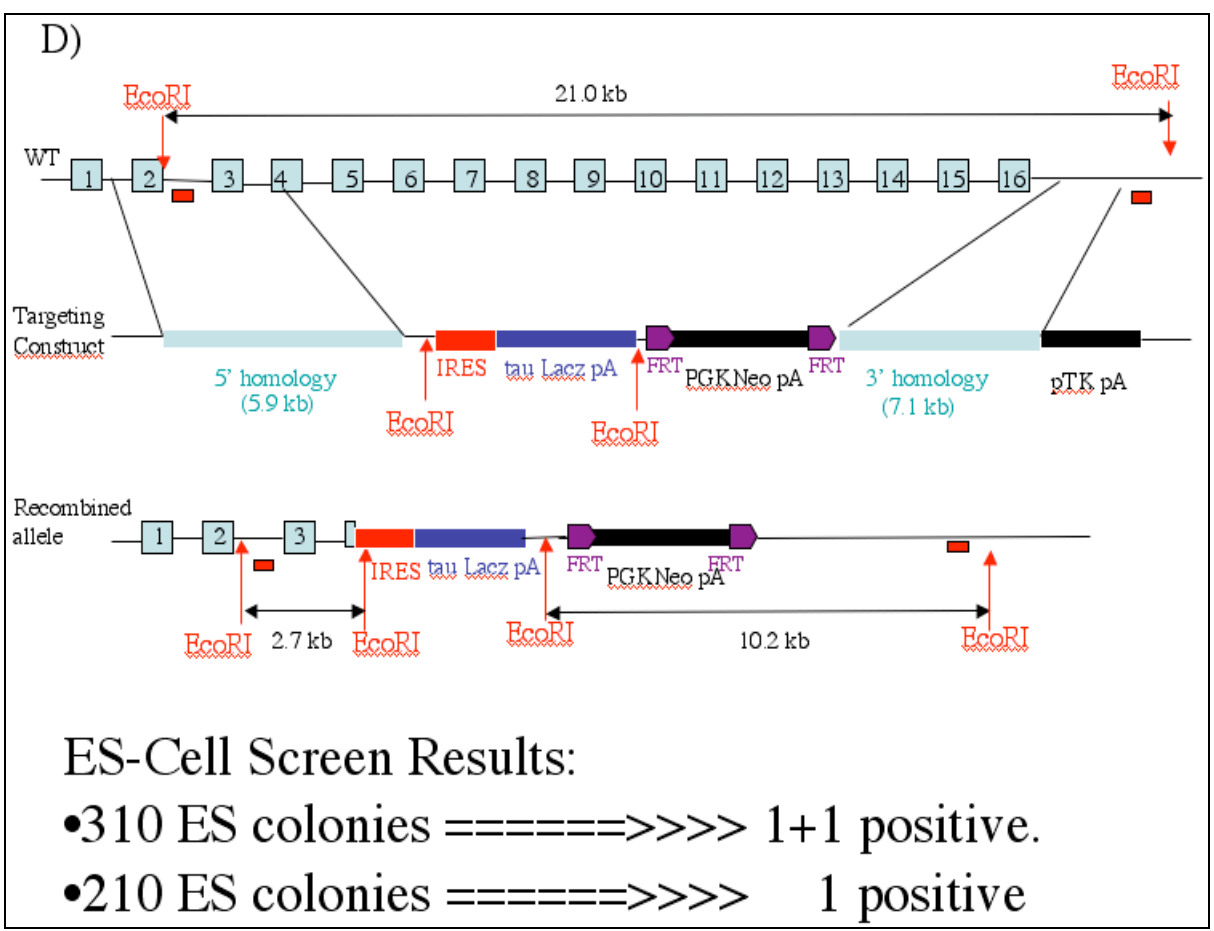




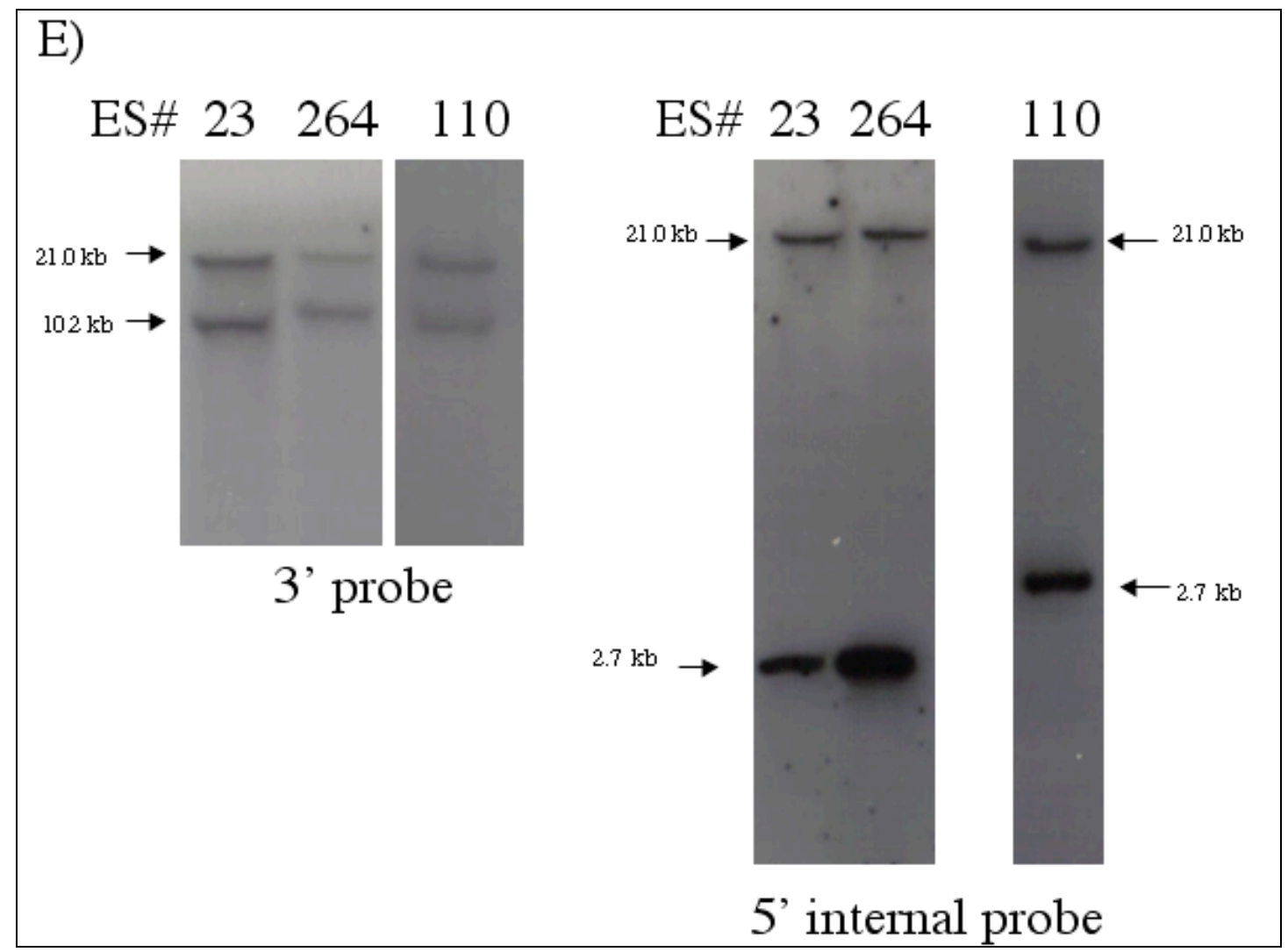

Figure 6. Knockout constructs and ES-cell screen results.

All the prepared targeting constructs for creating knockout mice are shown together with the representation of the wildtype (WT) allele and the recombined allele. All 16 exons of the unc $5 A$ gene are represented as blue boxes with lines between them that represent introns. EcoRI restriction sites, which are used for the Southern Blot Screen analysis are shown, together with the location of used Southern Hybridization probes (small red rectangles). The mentioned sizes between the EcoRI sites are the expected sizes for the Southern Hybridization results with the indicated probes. All the homology domains and used cassettes were shown as rectangles with the names written below them in the same color. The directions of FRT and LoxP sites are shown on the targeting constructs and the recombined allele representations. ES-cell screen results for each electroporation of each individual construct are mentioned under the figure of each corresponding construct. A: The first construct for creating a conventional knockout mice and its ES-cell screen results. B: The second construct for creating a conditional knockout mice and its ES-cell screen results. C: The third construct, which is a modified version of the second construct, and its ES-cell screen results. D: The fourth construct for creating a conventional knockout mice and its ES-cell screen results. E: Southern Blot Analysis results for the positive ES clones. EcoRI digested genomic DNA for each ES clone is analyzed via hybridization with $3^{\prime}$-external (left) and $5^{\prime}$ internal (right) probes. 


\section{Generation of chimaeras and screening for germline}

\section{$\underline{\text { transmissions }}$}

Chimaeras were first created from the two ES-clones (ES\#23 and ES\#264) by aggregation in our departmental facilities. From the first set of aggregations, 1 male chimera from ES\#23 and 6 chimaeras (4 male and 2 female) from ES\#264 were obtained. The resulting animals revealed only 10-20\% chimaerism. After several rounds of mating, these chimaeras did not give any germline transmission.

The same ES-cell clones were aggregated second time in order to produce more chimaeras. The second set of aggregations produced 2 male chimaeras from ES\#23, and none from ES\#264. These mice revealed only 20-30\% chimaerism. We also did not get any germ-line transmission from these chimaeras after several rounds of mating.

Then we decided to produce the new set of chimaeras via 'Blastocyst Injection' method, since this might increase our chance of obtaining chimaeras with higher germline transmission possibilities (personal communication with Franke U.). The blastocyst injection of both ES\#23 and ES\#264 clones gave us 4 male and 1 female chimaeras, which are all originated from the clone ES\#23. They revealed only 10-20\% chimaerism. Unfortunately, we also did not get any germline transmission from these chimaeras after several rounds of mating.

At this time, we obtained the new ES-cell clone (ES\#110) from the second electroporation of the last knockout construct (Figure 6D and 6E). We further tried to obtain new chimaeras from the previous ES\#23 clone and new ES\#110 clone via aggregation. The two trials of aggregation of these two clones did not produce any further chimaeras to analyze. Afterwards, we used these two clones for production of new chimaeras via the blastocyst injection method. As a result, we obtained 5 male and 7 female chimaeras for the clone ES\#23; and 15 male and 2 female chimaeras for the clone ES\#110. The chimaeras of ES\#23 had up to $30 \%$ chimaerism, whereas it was between $30 \%$ and $100 \%$ for chimaeras of ES\#110. After several rounds of mating for all the chimaeras originated from ES\#23, we did not obtain any germline transmission. However, 8 of the 15 male chimaeras of ES\#110 gave germline transmissions. The 2 female chimaeras coming from this ES clone were not analyzed. 


\section{General phenotypic investigations of unc5A knockout mice}

For initial characterization of a possible phenotype in the unc5A knockout mice, F1-hybrid matings were done by using the heterozygote animals produced during the germline transmission screenings. These animals had a mixed outbreed CD1 X inbred 129 backgrounds, since their mothers were the CD1 females used in germline transmission screen, and their fathers being the chimaeras that have their germ cells from the blastocyst-injected ES cells of a 129 background.

The distribution of the three expected genotypes from F1-matings was compatible with the Mendelian transmission ratio (25\% homozygote, $50 \%$ heterozygote, and $25 \%$ wildtype). Homozygote animals from these F1-litters were compared to their heterozygote and wild-type littermates. All homozygote mice, as well as heterozygote mice, were completely healthy and survived their normal life span without any observed abnormalities. Homozygote males and females are both fertile, and females can nurse their pups as good as their wildtype littermates.

In order to test for successful unc5A targeting and insertion of TauLacZ gene in the locus, the expressions of $u n c 5 A$ and TauLacZ were checked at the transcriptional level by RT-PCR. The deletion at the genomic DNA level has been validated earlier by southern blotting (Figure 6E) and by PCR-genotyping analysis from genomic DNA (Figure 7A). Total RNA samples were isolated from heads of PN-1 pups. RT-PCR analyses of these RNA samples showed the absence of unc5A mRNA and the presence of TauLacZ mRNA in the sample of the homozygous pup. The lower intensity of unc5A and TauLacZ band in the sample of the heterozygote pup (compared to WT and homozygote samples, respectively) is actually due to the presence of only one allele for each gene in the heterozygote state (Figure 7B).

In spite of the faithful expression of the TauLacZ reporter gene on the RNA level, I was unable to detect any LacZ staining in any trials with either embryonic or adult heterozygotes and homozygotes (see discussion). Appropriate positive and negative control embryonic or adult tissues were always included in the staining protocols. 


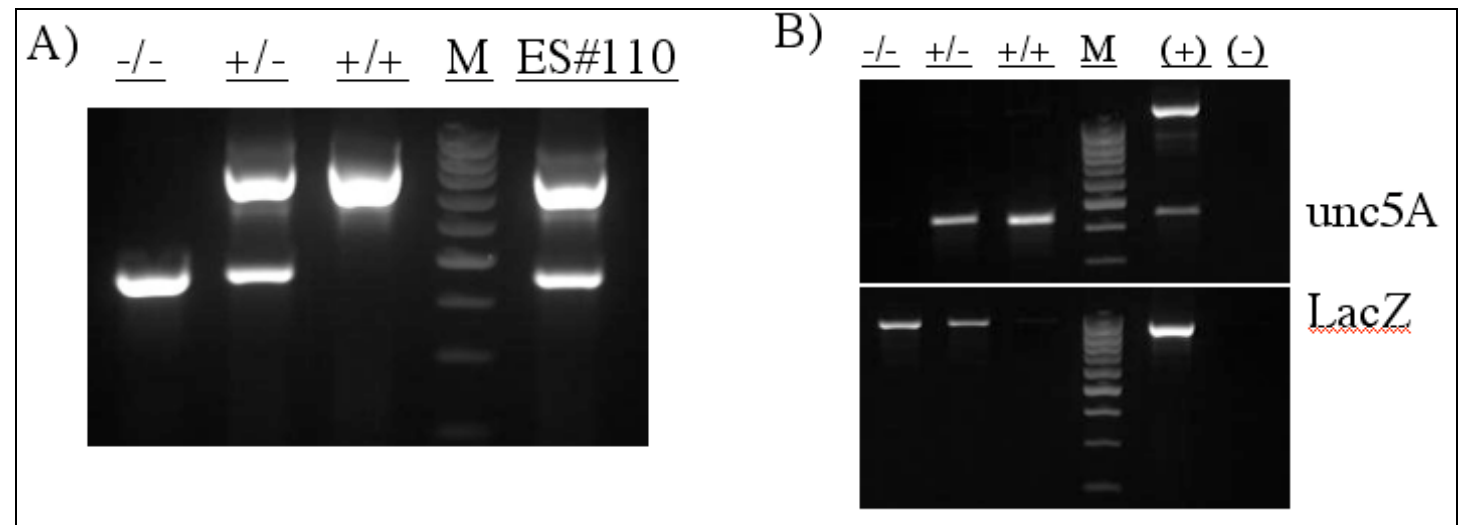

Figure 7. Proof for the deletion of unc5A on genomic DNA and RNA levels.

A: Genomic DNA samples from PN-1 pups were genotyped by PCR. Upper band (875 bps) corresponds to the wild-type allele, whereas the lower band (459 bps) corresponds to the deleted allele. Genomic DNA of the ES-cell clone \#110 is used as the positive control. B: Total RNA was isolated from the head samples of these three PN-1 pups, and checked by RT-PCR analysis using the ' $u n c 5 A$ ' specific primer-pairs (upper panel) and 'LacZ' specific primer-pairs (lower panel). Genomic DNA of one of the unc $5 A$-overexpression lines is used as positive $(+)$ control. (-) is the negative control. M, 100-bp DNA ladder. 
Since $u n c 5 A$ is expressed in the whisker pads (Engelkamp D., 2002), I compared the number and length of the whiskers in adult animals. There were no observed differences between homozygote and wild type littermates with respect to the number, organization or length of whiskers.

Because of the high level of unc $5 \mathrm{~A}$ expression in pyriform cortex and the migrating cells in rostral migratory stream (Figure 3A), I decided to check the olfactory behavior of unc5A knockout mice with simple behavioral tests. In these behavioral analyses, we were not able to detect any significant differences between wild type and homozygote litters.

Unc5A was identified originally in microarray analysis as a predominantly expressed gene in visual cortex. According to our ISH results, it had high level of expression not only in the visual cortex, but also in many regions of brain that is important for the visual system, like collicius superior, supraoptic area, and internal capsula in the posterior cortex (Figure 2A-E and Figure 3A-F). Due to such expression patterns of $u n c 5 A$ in several forebrain and midbrain regions that are important for the visual system, I started to check if the unc5A knockout mice have some visual problems. According to the simplest behavioral analysis methods for blindness, I found that some of the homozygous mice, but not all, were actually blind. The difference between these different homozygous mice could be due to having a mixed background, since it was also shown that the phenotype for unc5C knockout mice is dependent on the actual background of these mice (Burgess RW. et.al., 2006).

The isolated juvenile or adult brains of homozygous animals also showed some morphological abnormalities, like smaller cerebral hemispheres. But also this phenotype was not reproducible. Approximately $15 \%$ of the homozygous mice had this abnormality (to different extents) compared to their wild-type litters of the same gender. This discrepancy could also be due to the mixed background problem, just like the phenotype of blindness.

Because of encountering this mixed background problem, I decided to analyze the phenotypes of unc5A knockout mice in two different pure inbred backgrounds: 129 and C57/BL6-N. With this purpose, the chimaeras that gave germline transmission were mated first to 129 females. Since the ES cells were originally isolated from 129 background embryos, all the pups from these matings would have the pure 129 
background. For creating a cohort in order to analyze the phenotypes in C57/BL6-N background, these mice should be backcrossed into this background for at least 7 generations, which would give us (>99\%) pure C57/BL6-N background. The total backcrossing time would be approximately 1.5 year. Since these backcrossings are still continuing, I will not mention any kind of phenotypic analysis in this background.

129-inbred background has some disadvantages, like smaller litter size compared to other inbred backgrounds. This generally makes it difficult to obtain sufficient littermate pups or embryos of all three genotypes (especially wild-type and homozygote mutants together). Apart from this general disadvantage, it also brings some further difficulties especially for working on brain. First of all, most of the determined cortical region-specific marker genes were identified and shown in other backgrounds, especially in C57/BL6. Surprisingly, many of them do not respect completely the same regional territories in some other inbred backgrounds like 129. Most of these known marker genes are axon guidance or cell adhesion receptors, like ephrin receptors or cadherins, which has several differences in their expression patterns between 129 and C57/BL6 background mice. Second main problem of 129-inbred background for brain research is concerning the main axonal tract structures, like corpus callosum, corticocortical axon and even thalamocortical axon systems. In the brain of 129 background mice, the corpus callosum is much smaller, and the timing for the development of all these axonal systems differ compared to C57/BL6 mouse brain. Since most of the anatomical and molecular studies concerning these systems were done mostly in the C57/BL6 background, such results cannot be applied directly to the mutant mice of 129 background. However, the comparison of several genes' expression domains between wildtype and unc5A homozygous mice in 129 background could give us hints about any potential regionalization defects.

For preliminary phenotypic investigation in 129 inbred background, general health and behavioral aspects of the mutants were checked. All unc $5 \mathrm{~A}^{-1-}$ mice are completely healthy without any observed abnormalities. Both genders are fertile and the females can nurse their pups as good as their wild-type littermates.

The whisker pads of unc $5 \mathrm{~A}^{-/-}$mice were also analyzed compared to their wildtype littermates. There was no significant difference in terms of the length of whiskers or their organizations on the whisker pads. 
We also could not find any significant differences between homozygote and wildtype animals of 129 background for olfactory and auditory behaviors according to the applied simple behavioral assay systems.

Since some visual system phenotypes in mixed background was observed earlier, I tried to repeat these simple assays on the litters in 129 background. However, there were no observed differences between the homozygote and wild-type littermates in this inbred background.

The general morphology of cerebral cortex and other brain regions were then compared for any possible structural defects. There were no gross abnormalities or differences between the dissected brains of wildtype and homozygote littermates in terms of size or shape. Detailed histological examinations were done by Cresyl-Violet staining of paraffin sections from the brains of wildtype and homozygote littermates. For these analyses, I used at least 3 different pairs of animals for 3 different age groups: PN-0, PN-10 and PN-30. Some selected sections for PN-30 set are shown in Figure 8. The thickness of cortical plates and their lamination in all cortical regions within the anterior-posterior axis were observed under high magnification. However, no significant differences between homozygote and wild-type samples were visible. Also the corpus callosum and hippocampal structures were indistinguishable between these genotypes. Neither in diencephalic nor in midbrain or hindbrain structures, there was any significant abnormality in unc $5 \mathrm{~A}^{-/-}$brains.

After these histological analyses in three different stages, it was concluded that there are no big differences in the structure of neocortex or other brain regions due to the loss-of-function of unc5A gene in the 129 background. However, it is still necessary to analyze the marker gene expressions for both regionalization and lamination of neocortex in order to find out some possible minor defects in unc $5 \mathrm{~A}^{-/-}$neocortex. 


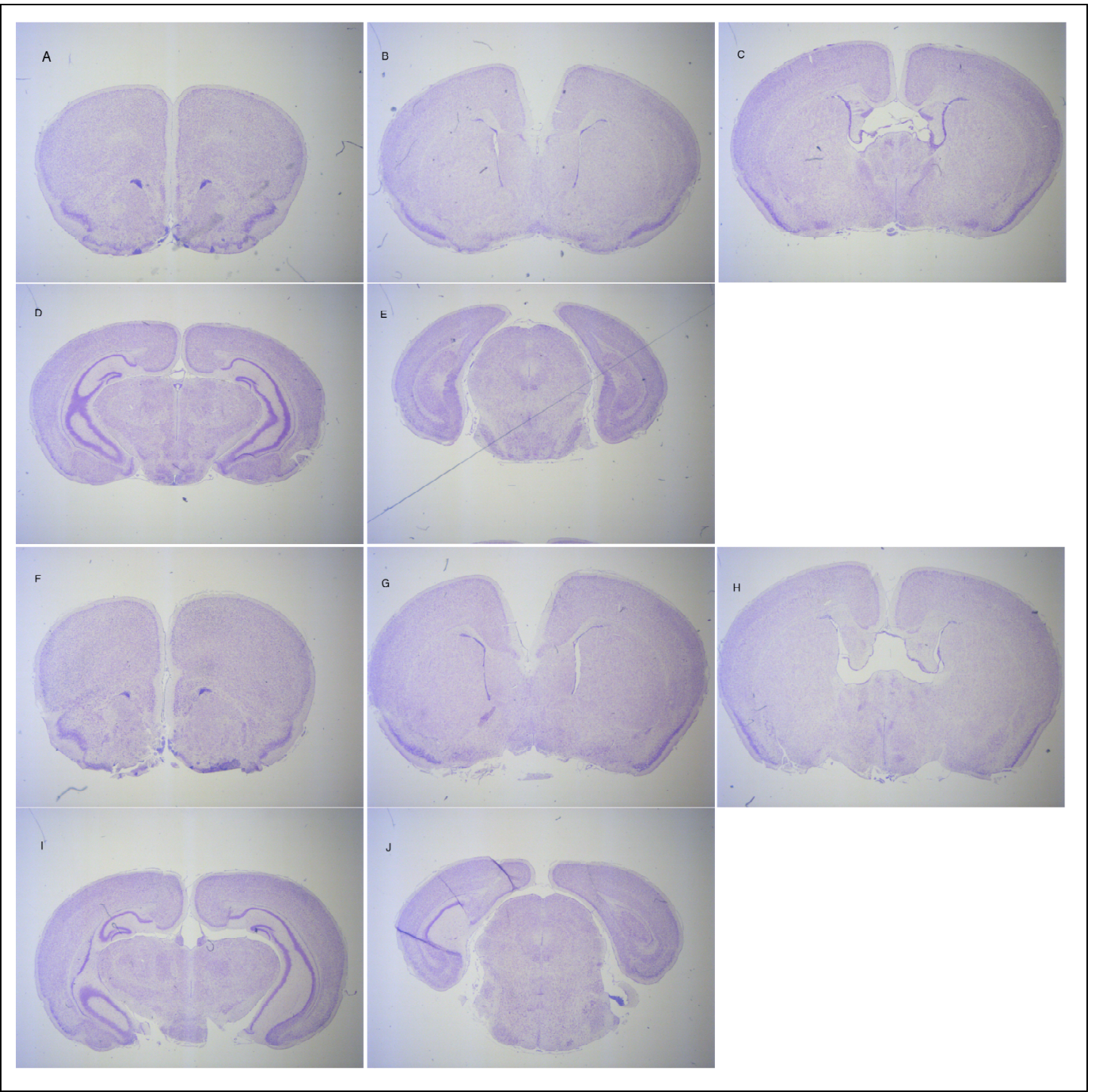

Figure 8. Histological comparison between wild type and homozygous PN-30 brains via Cresyl violet staining.

A-E: Wild-type samples with the order from anterior to posterior axis.

F-J: Homozygous samples with the same order. 


\section{Generation of 'Conditional Overexpression' construct and} screening for founder animals

In order to create the gain-of-function mutants for the " $\Delta$ exon6" splice variant of unc5A, a conditional ectopic-overexpression construct (Figure 9 A) was designed. The difference of the functions between the full-length and " $\Delta$ exon6" spliced variant of unc $5 \mathrm{~A}$ is currently unknown. Previously, we showed that the major form of unc $5 \mathrm{~A}$ expressed during embryogenesis is the " $\Delta$ exon6" spliced variant (Figure 5). It was therefore decided to ectopically over-express this spliced variant instead of the fulllength variant. An IMAGE clone (Genbank Acc: CA318180), which contains the fulllength cDNA sequence of " $\Delta$ exon6" spliced variant for unc $5 A$ gene, was obtained and cloned just upstream of an 'IRES-TauLacZ-pA' cassette. The 'TauLacZ' fusion gene that was placed on the 3'side of the unc5A cDNA sequence had an IRES sequence on its $5^{\prime}$-side. This would result in having bicistronic mRNA, that will produce both proteins (unc5A and TauLacZ) together. This reporter gene would be helpful for us to follow the unc5A-overexpressing cells and their axons. Then this unc5A cDNA fragment together with the 'IRES-TauLacZ-pA' cassette was cloned into the 3'end of the "conditionaloverexpression construct' obtained from the laboratory plasmid stock. This construct contained a composit CMV-IE and B-actin promoter (Sawicki JA. et.al., 1998), which is followed by $B$-actin intron, and floxed $e G F P$ sequences that contains several 'PolyA' signal sequences at the end. With the use of this construct, we aimed to create transgenic mice, which would constitutively express eGFP. After mating these transgenic mice with specific CRE-lines, we could delete the $e G F P$ sequences together with the polyA signal sequences via CRE-mediated homologous recombination only in the cells where there is a CRE expression (Figure 9B). This deletion would result in the overexpression of unc5A (" $\Delta$ exon6" spliced variant) protein together with the 'TauLacZ' reporter.

The microinjection of the prepared construct generated 65 founder mice, of which 3 were transgenic according to the genotyping results. These 3 founder mice were mated with C57/BL6-N partners, and the pups were checked for the uniform expression of eGFP. None of them gave the expected eGFP expression. This could be due to the genomic integration effect, in which they are silenced by some genomic sequences around the integration sites. 
Second trial of microinjection gave 63 further founder mice. Only one of them (\#55) was positive in genotype. The pups derived from this founder line gave the expected uniform expression of eGFP. This line was then backcrossed into C57/BL6-N inbred background.

While working with transgenic animals, it is important to have more than one founder lines in order to rule out that an observed phenotype is not an artifact of the specific site of integration of the transgenic construct. Because of this reason, the same construct was used in two further microinjections in order to have some more founder lines. After these microinjections, we obtained a total of 195 founder lines, in which 6 of them were genotypically positive. However, 4 of them did not succeed in mating or did not give the uniform eGFP expression in the analysis of their pups. Among this set of founder lines, two of them (\#11 and \#31) gave the uniform expression of eGFP in its descendants. These founder lines were also backcrossed into C57/BL6-N inbred background.

Uniform eGFP expression was detected in these 3 lines at embryonic stages and as well as in individual adult organs (Figure 9C). 


\section{A)}

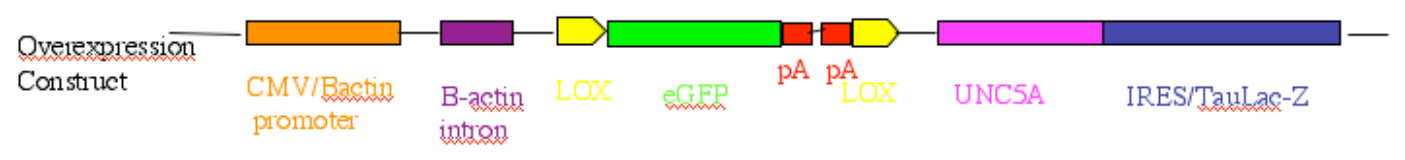

B)

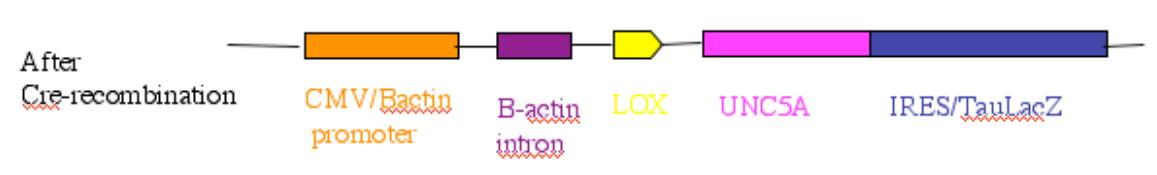

C)

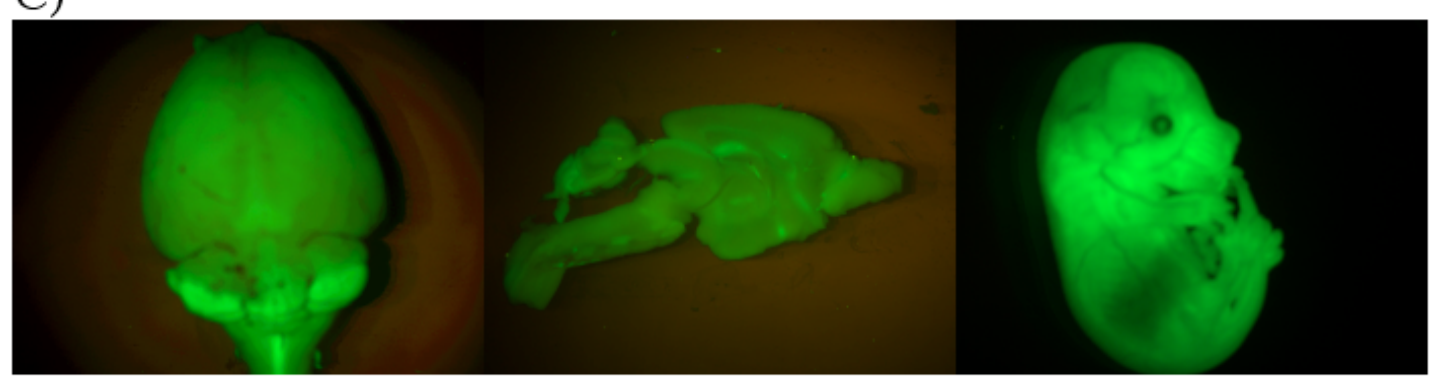

Figure 9. Generation of 'Conditional-Overexpression' line for unc5A.

A: The scheme of the unc $5 A$-conditional overexpression construct used for generating the transgenic lines. Functional cassettes are shown as rectangles with their names written below them in the same color. The 'LoxP' sites are shown with their orientations. B: The specific CRE-dependent recombination will remove the $e G F P$ and the polyA signal sequences and the expression of unc5A and TauLacZ genes will be switched on. C: Examples of uniform eGFP expression for unc $5 A$-overexpression lines in isolated adult brain (left), in sagittal section from an adult brain (middle), and E15.5 embryo (right). 


\section{Creation of double transgenics with Cre-lines}

Having a conditional overexpression line gave us the possibility to check the function of unc $5 A-\Delta$ exon6 in many different tissues or cells. Depending on the transgenic CRE-line that will be used, one could determine the spatio-temporal character of $u n c 5 A$ overexpression. With this possibility, we decided to create different double-transgenic animals via mating our overexpression line with different CRE-lines. The initial aim was to observe all these different double-transgenic animals for an apparent phenotype in order to analyze it further.

For this purpose, we used 2 different CRE-lines, which were already present in our department: (1) ECG-Cre, and (2) Nex-Cre. The results for our phenotypical observations concerning each will be explained in the next sections.

\section{Overexpression of UNC5A- $\Delta$ exon6 in developing neuroretina via}

\section{ECG-Cre}

The mouse ECG-Cre line ( $\alpha$-Cre) was selected because it expresses the CRE protein under the control of neuroretina-specific $\alpha$-promoter of pax6 gene. (Marquardt T. et al., 2001). As it was shown, the Cre activity appears quite early in all the retinal progenitor cells that will give rise to all 6 different retinal cell types: Retinal ganglion cells, horizontal cells, bipolar cells, amacrine cells, photoreceptor cells and Muller glia cells.

Unc5A is expressed in the central area of the developing E12.5 neuroretina (Engelkamp D., 2002). However, it is not expressed in the innermost layer, which is the birthplace of the first ganglionic cells. The expression of another unc5 gene (unc5B) starts earlier during development and it is expressed in the dorsal half of the neuroretina. On the other hand, the chemo-attractive Netrin receptor, $d c c$, is expressed in the innermost layers of the central neuroretina (Engelkamp D., 2002).

Non-overlapping expression of these three Netrin receptors during early development of the neuroretina could be important for sorting out the retinal ganglionic cell axons coming from different retinal sides in order to create the functional harmony. A disruption in this sequential order of retinal ganglionic cell axons would result in the 
false spatial representation of neuroretina in the corresponding nuclei of thalamus, and later on in the visual cortex. Similar situations were observed in the barrel cortex layer of the somatosensory cortex after the disruption of the whisker pads by removing some of the whiskers during embryogenesis or even during early postnatal life.

We hypothesized that we could interfere with this spatial representation of retinal ganglionic cell axons in the thalamus and visual cortex by ectopically overexpressing unc $5 \mathrm{~A}$ in all distal regions of neuroretina during early embryogenesis. We could then ask the question of how important is the affect of the thalamic influence during visual cortex development.

Since unc $5 A$ encodes a 'chemo-repulsive' Netrin receptor, we expected that all these different neuroretinal cells, especially retinal ganglionic cells, will have an impaired axonal guidance in which their exit into the optic nerve will be disrupted.

First of all, we checked the functional CRE-expression pattern via mating this ECG-Cre line with Floxed-ROSA line. LacZ staining of embryos as early as E12.5 showed that Cre activity is restricted only to the distal parts of the developing neuroretina (Figure 10). 


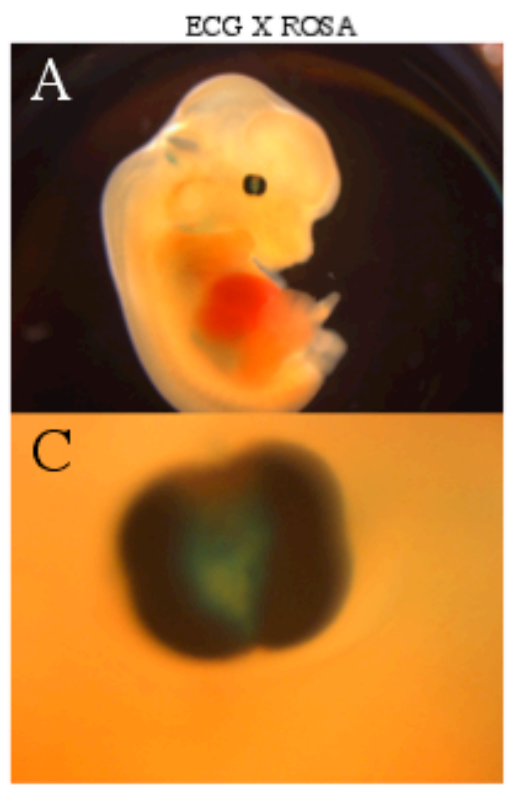

ECG X ROSA

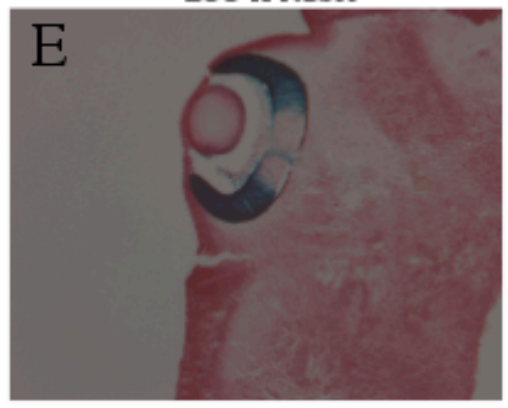

Figure 10. Expression pattern analysis for CRE activity of ECG-Cre transgenic mouse line.

ECG-Cre line is crossed with Floxed-ROSA line and E12.5 embryos were stained in whole-mount (A-D) or on $30 \mu \mathrm{m}$ sagittal sections ( $\mathbf{E}$ and $\mathbf{F})$ for LacZ expression. $\mathbf{A}$, $\mathbf{C}, \mathbf{E}$, and $\mathbf{F}$ are double transgenic embryos for both ECG-Cre and Floxed-ROSA transgene (ECG X ROSA); whereas B, and D are wildtype (WT) littermates. C and D are the high magnification pictures of eyes from embryos in $\mathbf{A}$ and $\mathbf{B}$, respectively. Neutral-Red staining is used for counterstaining in $\mathbf{E}$ and $\mathbf{F}$. WT samples are used in whole-mount stainings in order to distinguish the background stainings from the specific ones. 
Hybrid animals were produced by mating the unc $5 A$-overexpression line with the ECG-Cre line for the phenotypical investigations. All double transgenic animals were healthy and fertile in both genders. Starting from the age of PN-10, we analyzed the visual behavior of these double transgenic hybrids in comparison with each individual single transgenic and wildtype littermates. We observed the blindness phenotype only in some of these double transgenic animals (approximately 15\%), but not in all. We also observed that some of the single transgenic and even the wild-type littermates (approximately $10 \%$ of them) had blindness phenotypes. This phenotype cannot be therefore unambiguously linked to the ectopic overexpression of unc $5 A-\Delta$ exon6. The possible explanation for all this discrepancy could be due to the backgrounds of each transgenic line in use. ECG-Cre line within our department was kept in pure FVB background ( $>\mathrm{N} 15)$; and our unc5A-overexpression line was generated in B6CBA background, which was further backcrossed into C57/BL6-N background.

It was already announced by 'The Jackson Laboratory' that FVB inbred strain is known to be homozygous for the retinal degeneration allele Pde6 $b^{\text {rdl }}$ (JAX mice Data Sheet for FVB/NJ. The Jackson Laboratory; JAX Notes. Spring 2002). Since this allele is recessive, a retinal degeneration phenotype is not being expected in its heterozygote state, like in our hybrid animals. However, it cannot be predicted whether the presence of one wildtype allele of this gene (instead of two) could affect the loss-of-function or gain-of-function studies of other genes.

UNC5A-overexpression lines were generated via microinjection in B6CBA zygotes. It was also announced by 'The Jackson Laboratory' that this inbred strain has mutations at the Mitf locus that affects the eye size, pigmentation and the capacity for secondary bone resorption. (JAX mice Data Sheet for B6CBA. The Jackson Laboratory).

Initially, N1 generation of the unc5A-overexpression line was used for mating with the ECG-Cre . Afterwards, when we created the hybrid transgenic animals by using the N2 and N3 generation of unc 5A-overexpression line, the percentage of double transgenic animals that were blind increased from approximately $15 \%$ to $25 \%$ and $50 \%$, respectively. Since the C57/BL6-N inbred strain has no known defects concerning the eye development, we decided to bring all our unc $5 \mathrm{~A}$-overexpression lines' background into C57/BL6-N background (by at least 7 generations of backcrossing) prior to any further phenotypical analysis. During backcrossings into C57/BL6-N background, the 
contribution of this background in the progeny will gradually increase while decreasing the contribution of B6CBA background.

However, the blindness phenotype was still observed in approximately $10 \%$ of all of the single transgenic or wildtype littermates of hybrids generated by using N2 or N3 generation of unc5A-overexpression line. Since the ECG-Cre line was still in pure FVBbackground, also this mouse-line has to be backcrossed into C57/BL6-N background for the productions of next hybrids. As expected, no blindness phenotype was observed in any single transgenic or wild-type littermates of hybrids that were produced by using partially backcrossed (N3) ECG-Cre mouse-lines.

While these hybrid matings were in progress, I was also analyzing the embryonic development of neuroretina with histological methods. Due to the simultaneous expression of the reporter TauLacZ gene and unc5A, LacZ staining was performed on double transgenics. The staining pattern should allow us to follow any potential phenotype. However, we never obtained any kind of LacZ staining pattern in any of the analyzed double transgenic embryos for unc5A-overexpression and ECG-Cre. Since $u n c 5 A$ is a pro-apoptotic protein, one possible explanation for such an observation could be the death of all the cells that are overexpressing it. In order to see if the $\mathrm{CRE}^{+}$cells are still present in these double transgenic embryos, we designed matings to have triple transgenic embryos for 'unc5A-overexpression', 'ECG-Cre', and 'Floxed-ROSA' transgenes. There was less amount of LacZ staining in the triple transgenic E12.5 embryos (Figure 11C-D) compared to the double transgenic (ECG-Cre \& FloxedROSA) E12.5 embryos (in Figure 10E-F). However, their double transgenic littermates (unc5A-overexpression and ECG-Cre) did not give any similar type of stanings (Figure 11B). Together with the observations from the other used Cre line, which will be mentioned in the next section, it became clear that the TauLacZ expression was somehow not working. Possible explanations for it will be discussed in the next section.

In double transgenic animals for ECG-Cre and Floxed-ROSA, LacZ stainings were covering all the layers of distal neuroretina, and the axonal tracts from the ventral part of the distal neuroretina towards the exit point of optic nerve was visible (Figure $10 \mathrm{E}$ and F). In contrast, mostly the outer layers of distal neuroretina were stained for LacZ in the triple transgenic samples, and no axonal tracts from distal neuroretina towards the optic nerve was observed (Figure 11A, C and D). These results suggest a 
possible defect in the organization and/or functional connections of developing neuroretina. It will be still necessary to check the marker genes for all 6 different types of cells in the neuroretina in order to show a possible structural organization defect. Also specific axonal staining methods, like DiI-labeling, is crucial for proving the impairment of the axonal connections from all neuroretinal regions to the optic nerve.

Backcrossings of both unc5A-overexpression line and ECG-Cre line into C57/BL6-N background, as mentioned before, is important to obtain reproducible and significant phenotypical data concerning blindness. However, it should be mentioned here that such backcrossing would take approximately 1.5 year. Afterwards, it should become possible to analyze the neuroretinal phenotype and also the hypothesized developmental defects in embryonic and postnatal cerebral cortex. 

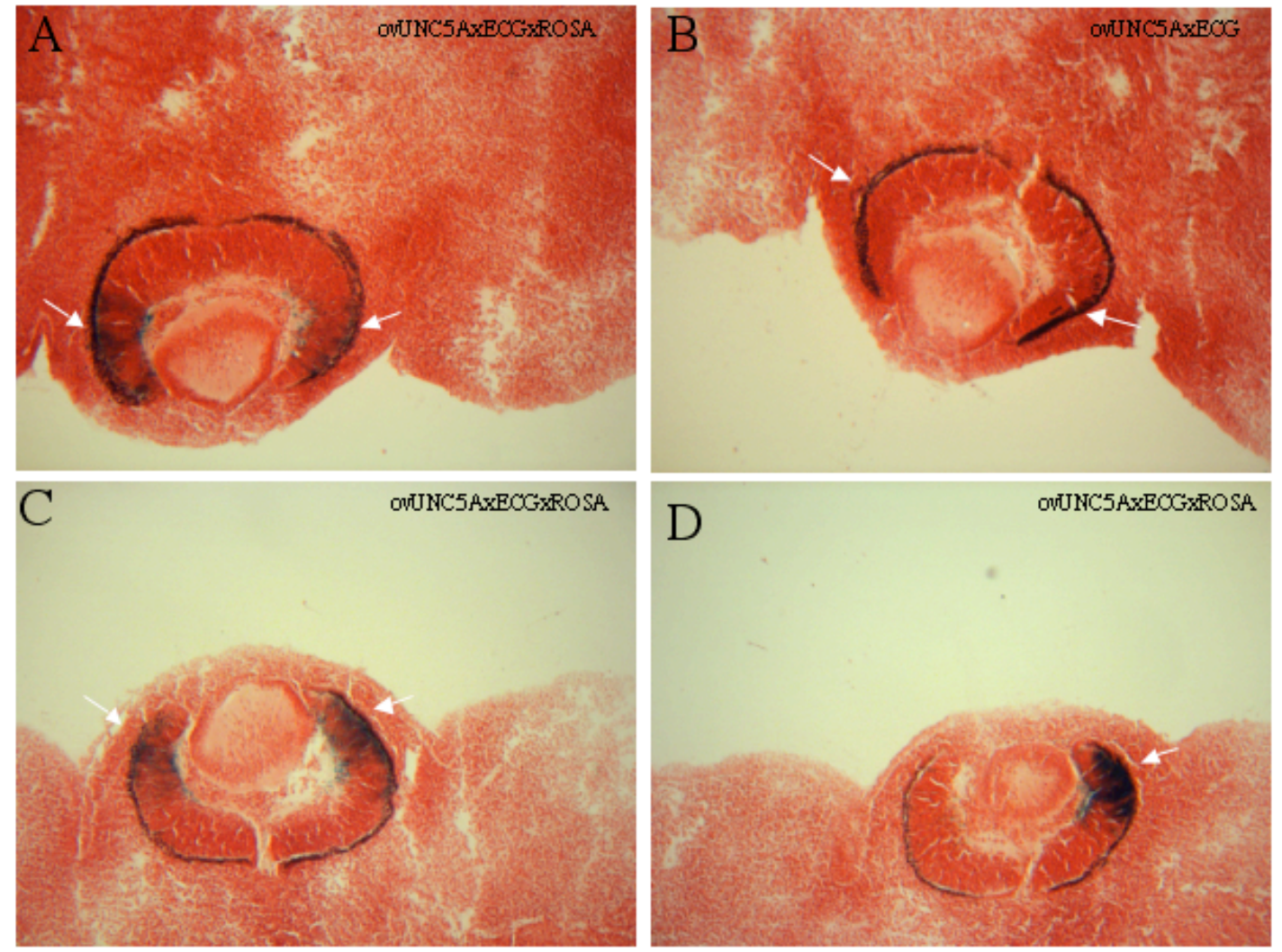

Figure 11. LacZ staining analysis of triple transgenic embryos (E12.5) for unc 5 A-overexpression, ECG-Cre, and floxed-Rosa transgenes. A, C, and D are different triple transgenic embryos (ovUNC5AxECGxROSA), whereas $\mathbf{B}$ is positive only for ECG-Cre and unc5A-overexpression transgenes (ovUNC5AxECG). A and $\mathbf{B}$ are $50 \mu \mathrm{m}$ sagittal sections; $\mathbf{C}$ and $\mathbf{D}$ are $30 \mu \mathrm{m}$ sagittal sections. White arrows are showing the distal parts of neuroretina where there is Cre expression. Counterstaining is done for all with Neutral-Red. 


\section{Overexpression of unc5A- $\Delta$ exon6 in Neocortex and Hippocampus} via Nex-Cre

Unc5A is predominantly expressed in visual and olfactory cortex regions of cerebral neocortex, as it was mentioned before. The specific expression pattern of unc $5 A$ in hippocampus was also previously shown for E14.5 (Figure 2) and E18.5 (Figure 3). Nex-Cre mouse line was used to overexpress unc $5 A$ in the post-mitotic neurons of all neocortical regions and also in the hippocampal pyramidal neurons. Such experiments would allow us to test 3 different hypotheses. (1) The ectopic overexpression of unc5A in the regions where it is not normally expressed could prevent or impair the axonal connections of the neurons belonging to these neocortical regions, assuming that the Netrin-dependent chemoattraction is important for them. (2) During the 'pathfinding' process of axons, they switch on or off specific repulsion or attraction mechanisms in order to respond to the same axon guidance cues along their path in a different manner. By continuously overexpressing the Netrin-dependent chemorepulsive receptor, unc5A, in the neurons where it is already being expressed, we could understand the significance of these 'switching on/off' mechanisms for the axonal pathfinding of several systems. (3) Hippocampus plays a crucial role in learning and memory functions of the brain. Its connections (mainly via the pyramidal neurons) with different regions of neocortical tissue are vital for these functions. Overexpressing the chemorepulsive axon-guidance receptor, unc5A, could disrupt some of these axonal connections, which require the Netrin-dependent chemoattraction mechanism.

We therefore used the Nex-Cre mouse line, a Nex-knockout / Cre-knockin line (Goebbels S. et.al., 2006). It was recently published that the Cre expression starts around E11.5 and marks the pyramidal neurons and dentate gyrus mossy and granule cells. Proliferating neural precursor cells in the ventricular zones, interneurons, oligodendrocytes, and astrocytes do not express CRE protein.

Initially, the functional CRE expression was checked by mating the Nex-Cre line with the reporter Floxed-ROSA line. As seen in figure 12, LacZ staining is specifically in the dorsal telencephalon and spinal cord of E13.5 embryos. More detailed Cre expression pattern for Nex-Cre mouse line was already published (Goebbels S. et.al., 2006). 
NEX-CREX ROSA

A

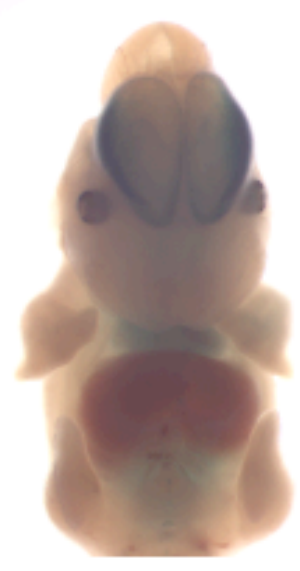

C

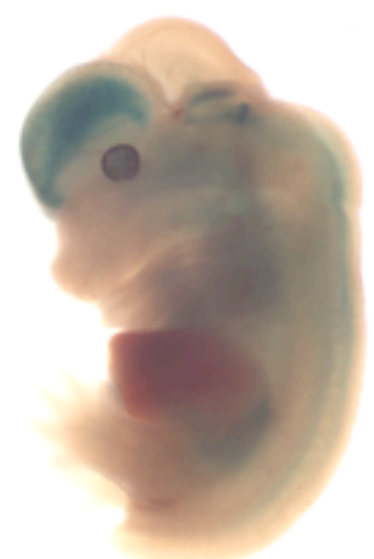

WT

$\mathrm{B}$

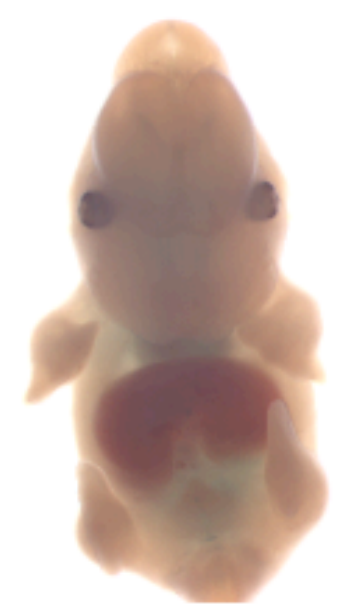

$\mathrm{D}$

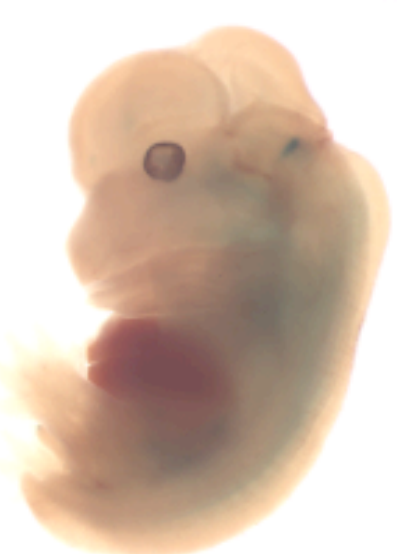

Figure 12. Functional CRE expression by the Nex-Cre transgenic mouse line.

Nex-Cre line is crossed with Floxed-ROSA line and E13.5 double transgenic (A and $\mathbf{C}$ ) and wildtype (B and $\mathbf{D}$ ) embryos were stained for LacZ in whole-mount. WT samples are used in this staining in order to distinguish the background stainings from the specific ones. 
The unc5A-overexpression line was mated with the Nex-Cre line for phenotypical investigations. Both male and female double transgenic animals were healthy and fertile. I tried to observe the postulated axonal guidance defects during the development of neocortex and hippocampus via the usage of TauLacZ reporter gene, which should be overexpressed together with unc5A. However, none of the LacZ staining trials for embryonic or adult stages gave any signal. In order to rule out the possibility that the conditional overexpression system is not working, I verified the overexpression of unc5A and TauLacZ at the RNA level by RT-PCR (Figure 13A). Hybrid pups were produced by mating the Nex-Cre line with mice from all three $u n c 5 A$-overexpression lines that we have. Total RNA samples were isolated from the brain samples of PN-1 pups from these hybrid matings and the expression of unc5A and TauLacZ were confirmed by specific RT-PCR analysis (Figure 13). Expression level of unc5A (' $\Delta$ exon6' spliced variant) was increased in double transgenic animals compared to the single transgenic Nex-Cre brains, which has only the endogenous unc5A expression. Expression of TauLacZ is detected only in double transgenic pups, as expected.

According to our conditional overexpression system, expression of CRE causes the removal of the eGFP expression (compare Figure 15A with $\mathrm{B}$ and $\mathrm{C}$ ). As expected, the $e G F P$ expression in the forebrain region of triple transgenic animals disappeared. These results confirmed that our conditional overexpression system works at least at the transcriptional level, although we cannot still rule out the possibility of a mistake in the translation of unc5A-tauLacZ bicistronic mRNA. In order to check this possibility, the construct used to produce these overexpression lines was transfected into a special bacterial strain, which expresses the CRE protein. The Cre-recombined version of the construct was isolated and used to transfect Neuro2A cells in parallel with the original construct. We had eGFP signals but not LacZ signals in the set with the original construct, whereas eGFP signals disappeared with the appearance of strong blue LacZ staining for the recombined version of the construct (Figure 14). In conclusion, it is clear that Cre-dependent deletion of $e G F P$ sequences occur correctly and consequently $u n c 5 A$ and tauLacZ genes/proteins are overexpressed. The real reason for not observing any tauLacZ staining in the mouse experiments is currently not known. 


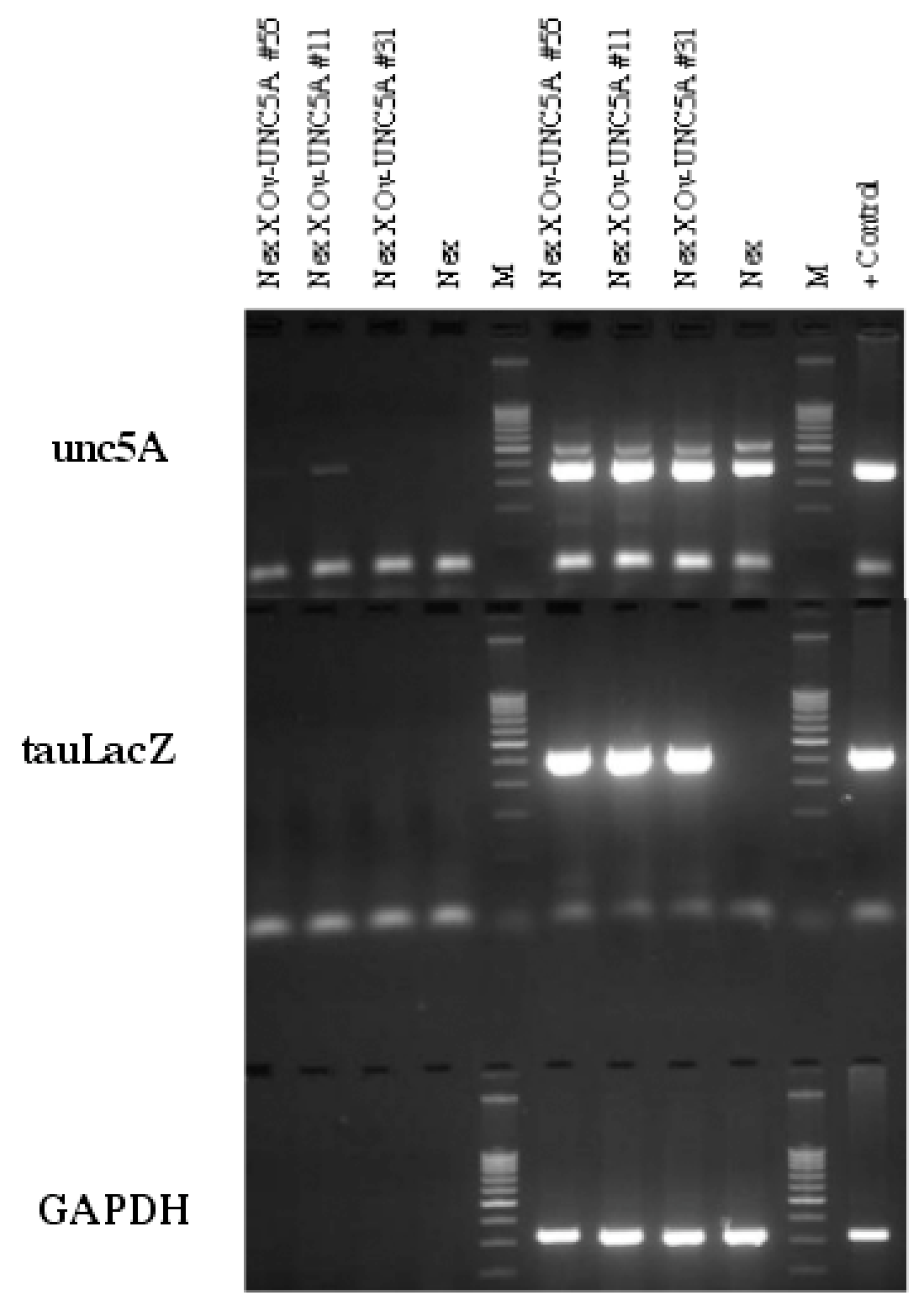

Figure 13. RT-PCR analysis for confirming the overexpression of $u n c 5 A$ and TauLacZ.

All three different unc5A-overexpression lines (\#55, \#11 and \#31) were mated with Nex-Cre line. Brain samples were dissected from double transgenic PN-1 pups of each mating together with Nex-Cre single transgenic littermates and total RNA were isolated from each. RT-PCR analyses were done with specific primers for unc $5 A$ and TauLacZ genes. First four columns on the left are the RT(-) controls. GAPDH-PCR is used for normalization against the density differences in synthesized cDNA samples. Primer pairs used for ' $u n c 5 A$ ' amplifies both spliced variants. The upper band with lower intensity is the full-length variant, whereas the lower band is ' $\Delta$ exon6' spliced variant. $\mathrm{M}$ is the 100 -bp DNA ladder as size marker. 

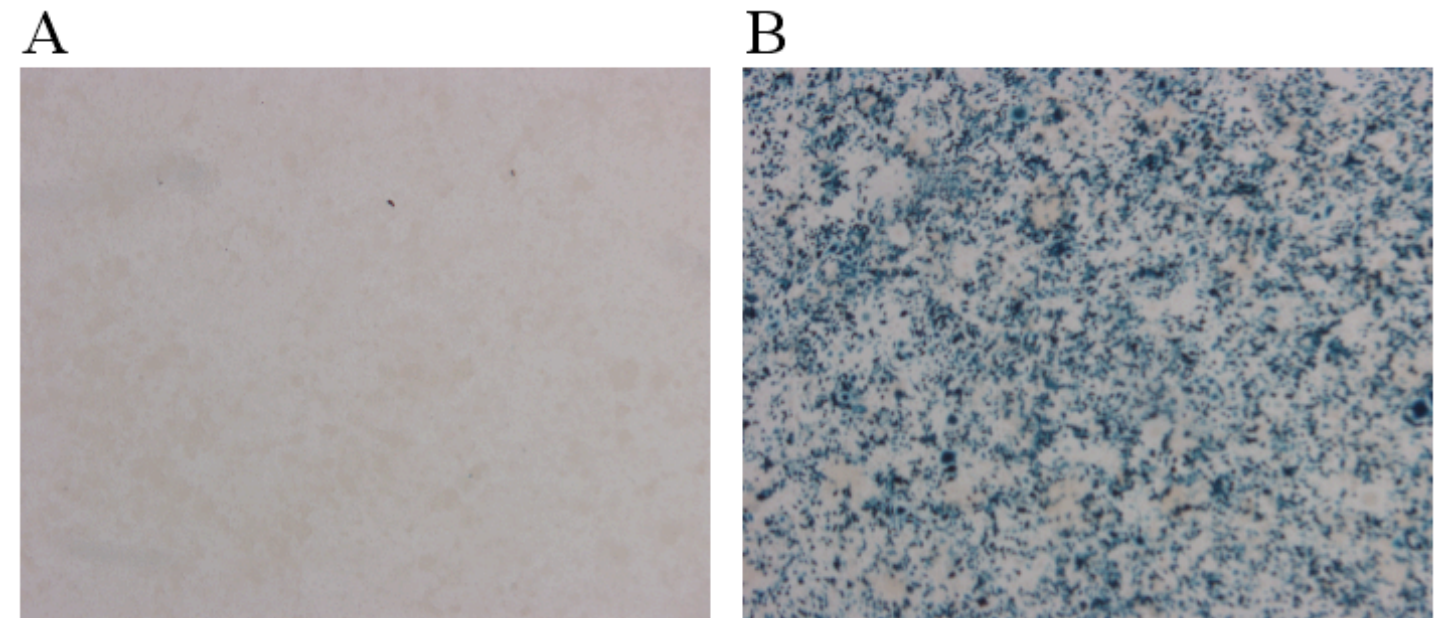

Figure 14. LacZ staining of transfected Neuro2A cells.

A: The original 'unc5A-Aexon6 conditional overexpression construct' (Figure 9A) was used in transfection. B: The CRE-recombined version of the same construct (Figure 9B) was used for transfections. 48 hours after transfection, the cells are fixed and stained for LacZ activity. 
I then tried to observe a phenotype on the morphological level by creating triple transgenics via mating our unc5A-overexpression lines with Nex-Cre /Floxed-Rosa double transgenic mice. According to our results of E15.5 embryos, the LacZ expressing zone in the triple transgenic ones (Figure 15D and $\mathrm{G}$ ) are on the same locations as the double transgenic Nex-Cre / Floxed-ROSA littermates (Figure 15F and I). However, the intensity of the staining for the triple transgenic embryo is much stronger than the other.

Since this study with Nex-Cre line was started quite recently, sufficient data has not been obtained yet to observe the hypothesized phenotypes. Detailed histological analyses of the cerebral cortex and the behavioral analysis for learning and memory have to be done in order to understand the functional role of $u n c 5 \mathrm{~A}$ gene in cortical development. 


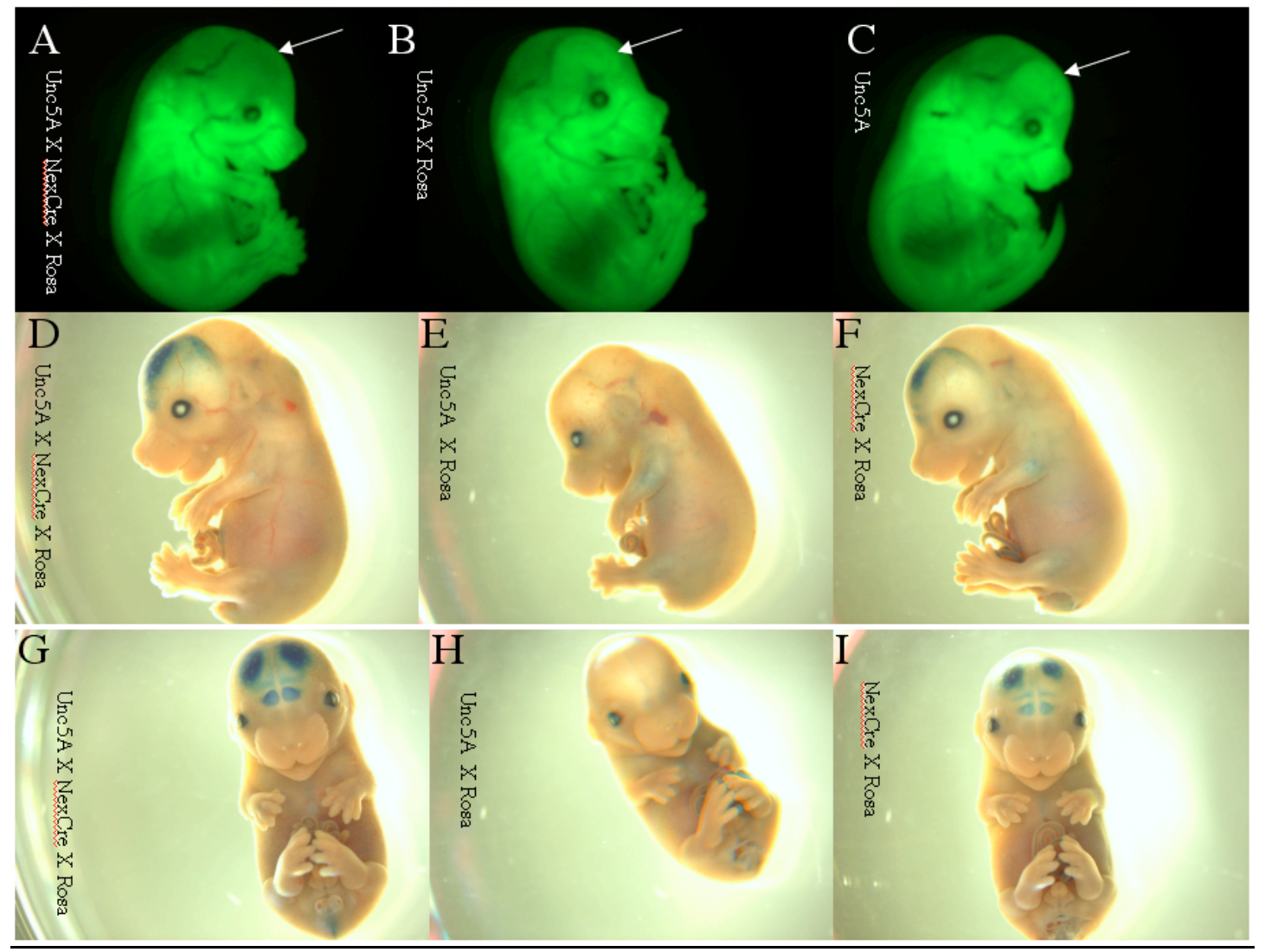

Figure 15. GFP and LacZ Analysis of triple transgenic embryos (E15.5) for unc $5 A$-overexpression, ECG-Cre, and floxed-Rosa transgenes.

$\mathbf{A}, \mathbf{D}$, and $\mathbf{G}$ are the triple transgenic embryo (unc5A X NexCre X Rosa); B, E, and $\mathbf{H}$ are the double transgenic embryo (unc5A X Rosa); $\mathbf{C}$ is the single transgenic embryo (unc5A); $\mathbf{F}$ and $\mathbf{I}$ are the double transgenic embryo (NexCre X Rosa). A-C are showing the eGFP expression with arrows indicating the forebrain region. D-I are the whole-mount LacZ staining results. 


\section{DISCUSSION}

During the 3.4 billion years of evolutionary history, the need for sensing the environment and regulating the responses against the conditions outside always remained a crucial function for all living organisms. Parallel to the evolutionary development of more advanced organisms, the neural systems responsible for this vital response functions, also developed further complexities. In this sense, the cerebral neocortex of the evolving mammals acquired improved sensory, learning, and decisionmaking activities during evolution. Compared to the early ancestors of mammalian brain in first fishes or reptiles, mammalian cerebral cortex is much bigger in size, highly regionalized and functionally more advanced in terms of consisting neural networks.

In this highly regionalized mammalian neocortex, different cortical areas have different functional responsibilities. Primary sensory cortices for visual, olfactory, auditory and somatosensory systems collect and interpret the inputs from the sensory organs, whereas secondary sensory cortices (association areas) for these systems and the motor cortices further interpret the inputs and do the 'decision-making' in association with the limbic system of the brain. During all these activities, they also create a 'memory' by the process of learning in order to advance their future functional activities. The required functional connections for these advanced neural systems are maintained by the long axons of specific neurons.

In order to gain a better understanding for the structure and functions of neocortex, it is very important to elucidate the mechanisms of its development. In this respect, the main questions to be answered are: (1) how does this highly complex structure develop from a pool of identical neuroepithelial progenitor cells; and (2) how are the correct neural connections established for its proper functioning.

Concerning the structural development of the neocortex, two different aspects should be considered: the regionalization of the cerebral neocortex, and the lamination of its cortical plate.

There are two hypotheses trying to explain the mechanisms of neocortical regionalization. 'protomap hypothesis' (Rakic P., 1988) suggests that the 
regionalization of neocortex is established via the intrinsic mechanisms, which are based on the brain inductive signaling centers and their secreted morphogens. According to this model the fate of cells in different cortical regions are already determined before the arrival of TCA inputs to the neocortex in a region-specific manner. On the other hand, 'protocortex hypothesis' (O'Leary DD., 1989) suggests that the regionalization of neocortex is solely determined and maintained via the information input of the incoming TCA. After a long debate between these two models, the current view on this topic combines both these hypotheses by suggesting that the initial structural regionalization is established through the intrinsic "protomap" mechanism, however, the functional regionalization and the maintenance of structural regionalization depends on the TCA information flow, as suggested by the “protocortex” hypothesis. (Rubenstein JL. et.al., 1999; O’Leary DD. and Nakagawa Y., 2002).

Regarding the second aspect of neocortical development, which is the lamination for creating the 6-layered cortical plate, our current view is based on two complementary models. The first model, 'Inside-first, Outside-last' mechanism, explains how this 6-layered structure is being established with different cell type compositions in each specific layer of cortical plate. According to this model, early born post-mitotic neurons are located within the inner layers, whereas the late-born ones migrate over the previous ones and create the outer layers of the cortical plate. On the other hand, the second model explains that the developmental time of their birth makes the neurons of different layers distinct, although they are born from the same pool of the progenitor cells (McConnell SK. and Kaznowski CE., 1991; McConnell SK., 1995).

So far, we do not have almost any clear understanding about the mechanisms of the 'functional' development of the neocortex. It is obvious that the functionality of neocortex relies on the establishment of the correct synaptic connections between different neocortical regions and also between these regions and other necessary brain structures. However, the nature of the developmental mechanisms regulating these highly complex and crucial processes is still unclear. It is therefore important to identify and functionally characterize the involving 'axon guidance' systems in order to gain a better understanding of synaptogenesis. 
In our group, we attempted to identify region-specific genes in developing neocortex by using microarray chip analysis with the aim of identifying potentially important genes for neocortical regionalization and lamination. In this study, I analyzed the microarray data for E14.5 stage and found a novel gene with predominant expression in the visual cortex. Identification of this gene (unc5A) was done both in silico and experimentally. In order to understand its potential role during the development of neocortex, its loss-of- and ectopic gain-of- function mutants were created and analyzed.

\section{Microarray analysis of E14.5 mouse cerebral cortices determined} the sets of genes showing region-specific expressions among different

\section{cortical regions}

Thalamocortical axons (TCA) reach to specific cortical layers of different neocortical regions around the developmental age of E15-E16. Therefore, it is important to determine the region-specific gene expressions within the neocortex at an earlier developmental stage (E14.5) to analyze the effect of intrinsic mechanisms during the neocortex regionalization. As proposed by the 'protomap hypothesis', the differences among different neocortical regions at E14.5 are completely under the regulation of intrinsic mechanisms. With this purpose, the large-scale comparative gene-expression analyses were done for E14.5 neocortical regions by using the Affymetrix DNA microarray chips.

The data set for this analysis contained 216 genes with a predominant expression in one of the analyzed cortical regions compared to at least one other region according to our selection criteria of " max-fold change $>3.0$ ". Majority of these genes showed the region specificity for hippocampus and somatosensory cortex (Figure 1A). This distribution actually fits to the expectations based on our current knowledge about the development of cerebral cortex. Hippocampus belongs to the evolutionarily older part of the cerebral cortex compared to the neocortical regions. Therefore, the sets of genes important for its development and for providing region-identity could be different than the genes responsible for neocortical areas. We observed many genes in our data set, 
which shows expression only in hippocampus but not in any neocortical regions, like $k l h d c 8 A$ (Figure 2F-J). On the other hand, we also observed several genes with different level of expressions in different neocortical regions, but no expression in hippocampus, like satB2 (Figures 2K-M and 3G-L). These observations also support the idea of having different sets of genes for evolutionarily distinct cortical regions.

At E14.5, the developmental stage of somatosensory cortex is at a much more advanced level than that of visual or motor cortex. It can be expected that the number of genes, which are specific for the differentiated-states of the different post-mitotic neurons within the developing neocortical plates, would be higher in the regions where the development is more advanced. Accordingly, we had much more number of genes with a predominant expression in somatosensory cortex than in visual or motor cortices (Figure 1A).

During such an early stage of corticogenesis like E14.5, most of the cells in the analyzed cortical regions are the radial glia cells (progenitors of most of the neuronal cells and all the glial cells within the cerebral cortex) of the ventricular zone, neuronal progenitor cells within the subventricular zone, and the early-born post-mitotic neurons of the inner cortical layers. Since it is expected by the 'protomap hypothesis' that different types of differentiation mechanisms should be switched on in different neocortical areas, it is expected to observe differential expression of region-specific transcription factors and signal transduction molecules. The categorization of all the known genes within our microarray data set according to their known or predicted molecular functions, actually confirmed this expectation (Figure 1B). Transcription factors and signal transduction molecules were represented as $18 \%$ and $29 \%$ of all known genes detected, respectively. The other large set of known genes detected by this microarray analysis contained the ones for extracellular matrix (ECM) proteins, celladhesion, cytoskeleton, and axon guidance molecules. Unlike the transcription factors or signal transduction molecules that are important for initiating the specific differentiation mechanisms, these other set of genes are most probably representing the differences of the differentiated or specialized cell lineages within different neocortical areas.

Furthermore, in silico prediction of the genes for the 100 ESTs within the dataset identified many novel genes with different functions, like mitochondrial or cytoplasmic metabolism. There were also some predicted genes for extracellular matrix proteins, 
transcriptional or translational regulators, axon guidance and signal transduction molecules. Besides the ones for which we could predict a protein function, there were also several other novel genes either with no predicted functions or even with no openreading frames.

Such region-specific expressions of genes for cellular metabolism could also be an indicative of the intrinsic mechanisms of 'protomap hypothesis'. It has been postulated that the morphogenic gradients created by the brain inductive signaling centers switches on distinct differentiation mechanisms in different neocortical regions, which in turn leads to different cell types within the different neocortical areas of E14.5 mouse cortex. Therefore, observing differential expression of the genes for cellular metabolisms could indicate the differences of cell types among these neocortical areas.

It is always important to verify and validate the microarray data by real time RTPCR or ISH analysis of some of the selected genes. It was decided to perform ISH, because this is a much better method to detect and show gradient-like expression pattern of any gene in tissues or organs. For 21 selected genes from our dataset, the ISH analyses were done on E14.5 brain sections. Three examples are shown in Figure 2. Same tendency of fold-changes in the microarray dataset were observed for almost all genes tested by ISH analysis. This confirmation of the microarray data suggests that the results of our large-scale analysis are trustable.

\section{Expression analyses and in silico identification of candidate genes}

\section{for functional studies}

The 21 genes, mentioned above, were selected for identifying a novel candidate gene and to characterize its functional role during the neocortical regionalization. The initial selection of them from the whole microarray dataset was based on their predicted molecular functions. The genes encoding for novel transcriptional or translational regulators, signal transduction molecules, and axon-guidance or cellular adhesion molecules were specifically chosen because these kinds of molecular functions could possibly be important for the determination and establishment of the neocortical regionalization. 
To further reduce the number of the candidate genes, additional selection from these 21 genes was done based on the results of their ISH analysis performed on E14.5 mouse brains, which was initially done to confirm the microarray data. These ISH analyses also showed the expression of these genes in other regions of the E14.5 brain that were not included in the microarray analysis (Figure 2).

According to the 'protomap hypothesis', the regionalization of neocortex is regulated by the morphogenic gradients secreted by the brain inductive signaling centers. These morphogen gradients are sensed and then interpreted by the neural progenitor cells via expressing specific transcription factors, like Pax6, Emx2, or Lhx1, to switch on the distinct differentiation mechanisms. During this process, a gradient like expression of these transcription factors appear mimicking the morphogenic gradient. Therefore, it is hypothesized that the genes that are important for the determination of the regional-identity within a developmental axis should show a gradient of expression on these same axes. Based on this hypothesis, the genes with specific gradients of expression throughout the different developmental axes of cerebral cortex were selected. On the other hand, the genes with high levels of expression in most of the midbrain and hindbrain structures were eliminated from the list, because the functional studies for this type of genes by creating conventional loss-of-function mutants could be difficult to analyze for their roles in neocortical regionalization. After this step of selection, there were only 3 genes left: AU-4 (satB2), AU-16 (klhdc8A), and AU-18 (unc5A).

AU-16 was also eliminated later because of two reasons. First of all, it was identified as a gene predominantly expressed in hippocampus by both microarray and ISH analysis. Moreover, no expression of this gene was detected in the neocortex (Figure 2F-J). Therefore, it can be an important gene for the development of hippocampus but not for the regionalization of neocortex. Secondly, it encodes a protein that only contains $4 \mathrm{KELCH}$ motifs (Figure 4B). Therefore, it is not possible to predict a certain function for this protein, since Kelch motif is important for any kind of protein-protein interactions.

ISH analyses for the other two genes were done on E18.5 mouse brain sections in order to see their expression patterns within the developmentally older brain, where the principal neocortical regionalization has already been established (Figure 3). 
The expression of AU-4 (Satb2) in E14.5 brains was restricted only in basolateral and somatosensory cortices (Figure 2K-M). However, by E18.5, high levels of expression were observed in cingulate and visual cortices in addition to the basolateral and somatosensory cortices (Figure 3G-L). Interestingly, no hippocampal expression was detected for E14.5 or E18.5 stages. This gene was also not expressed in any midbrain or hindbrain structures. These expression patterns suggest that Satb2 is a neocortex-specific gene. According to the gene-prediction results, it encodes a protein with two CUT domains and one homeodomain (Figure 4C). Both types of these domains are the DNA-binding domains found in the CUT-family of transcription factors. So, being a transcription factor with highly restricted expression for and within the neocortex makes this gene a perfect candidate to analyze its function during neocortical development and regionalization. However, the loss-of and gain-of-function mutants for this gene have already been created (personal communication, Tarabykin V.).

AU-18 (unc5A) was identified by the microarray analysis as a gene with predominant expression in the visual cortex. According to the ISH results on E14.5 brain, it is expressed in the cortical plates of visual, basolateral, and pyriform cortices (Figure 2A-E). There are also restricted expressions of unc5A in several forebrain structures, like internal capsula, epithalamus, supraoptic area, dorsomedial hypothalamic nucleus and central nucleus of amygdala. The expression pattern on E18.5 mouse brain sections showed even more striking anterior-low and posterior-high gradient of expression (Figure 3A-F). High levels of restricted expression pattern in visual and pyriform cortices were detected within the neocortex. The specific expressions within the rostral migratory stream, and some hippocampal areas like CA3 and dentate gyrus appeared within this developmental stage. In addition to the epithalamus and hypothalamus, there was also remarkably strong expression in dorsal thalamus, but not in ventral thalamus. Many other important brain structures for the visual system, like medial preoptic nucleus and collicius superior revealed the unc $5 \mathrm{~A}$ expression.

Unc5A expression was detected in many different brain structures. However, these different regions of its expression are actually connecting the different brain structures important for the visual and also the olfactory systems. Therefore, we postulated that unc $5 A$ could play an important role for the development of these systems 
not only at the level of their related neocortical regions, but also including the rest of the structures within these systems. In our gene-prediction studies, this gene was identified as a chemorepulsive receptor for Netrin-dependent axon guidance mechanism. It had the highest homology to the mouse $\mathrm{rcm}$ (Rostral Cerebellar Malformation) gene, which is renamed later as $u n c 5 C$. It contains all the protein motifs common to all known UNC5 family of proteins: two Immunoglobulin (IG), and two thrombospondin (TSP) domains as extracellular part, single-span type of transmembrane domain, and ZU-5 and DEATH domains as intracellular part (Figure 4A). Because of its predicted function as a Netrin-dependent chemorepulsive receptor and its expression patterns in the developing mouse brain mentioned above, it is highly possible that unc $5 \mathrm{~A}$ is playing a vital role in structural and/or functional development of visual and olfactory systems via regulating the axonal guidance for the establishment of the necessary and correct synaptic connections. Since netrin-dependent axon guidance system is also believed to regulate some cellular migration processes, it is also possible that unc $5 \mathrm{~A}$ is important for radial or tangential migrations during the neocortical development. In order to test these hypotheses, I decided to identify and functionally characterize the roles of unc $5 \mathrm{~A}$ during neocortical development and regionalization within this study.

\section{$\underline{\text { Identification of unc5A gene structure and its spliced variants }}$}

By both 5'- and 3'- RACE analysis, the in silico predicted cDNA sequence of unc $5 \mathrm{~A}$ gene was confirmed. The genomic structure of this gene was identified bioinformatically by aligning its cDNA sequence with mouse genomic sequences (from CELERA, NCBI, and ENSEMBL genomic databases) in order to find the exon-intron structures. Possible spliced variants of unc $5 A$ expressed during embryogenesis were identified by RT-PCR analysis using several primer pairs covering different combinations of exonic sequences. The only identified spliced variant was ' $\Delta$ exon6', in which the $6^{\text {th }}$ exon was spliced out. The major form of $u n c 5 A$ expressed throughout embryogenesis is this ' $\Delta$ exon6' spliced variant (Figure 5). However, soon after obtaining these results for the identification of the unc $5 \mathrm{~A}$ gene and its spliced variant, these data were published by Engelkamp D. (2002). The proposed gene structures in this publication matched completely to our data. 


\section{Generation of unc5A loss-of-function mutants}

A classical approach to understand the function of a gene in biological processes is to eliminate the expression of that gene by creating a loss-of-function mutant and to observe the resulting phenotypes due to this genetic modification. Several such mutants have been generated for studying neocortical development. (Job C. and Tan SS., 2003; Zaki PA. et.al., 2003). Here, I first decided to characterize the role of unc5A during corticogenesis, by the loss of function mutagenesis approach.

For this purpose, initially a DNA construct was designed for the generation of a conventional type of knockout mice (Figure 6A). PCR-amplified 5'- and 3'-homology arms were placed in this construct for obtaining homologous recombination in ES cells in order to target all the exons encoding the intracellular domains of unc5A protein. TauLacZ fusion gene was introduced into the construct to replace the deleted region of unc $5 \mathrm{~A}$ gene after homologous recombination in order to serve as a reporter gene to mimic the endogenous expression of $u n c 5 A$. Unfortunately, no positive clones for the expected homologous recombination was obtained after screening 255 ES clones (Figure 6A).

It is known that some genomic loci are less prone to have a homologous recombination than others, although the reason for this is not clear. However, it is speculated that the level of compaction for the genomic DNA shows differences for different genomic loci, and the degree of compaction affects the access of the knockout constructs to the correct genomic region for homologous recombination event to take place. Although there was a total of approximately $10 \mathrm{~kb}$ homology region within our construct, no ES clone with the homologous recombination in the correct genomic loci was obtained. Therefore, in the next construct, the homology regions were further extended. It is also important for the recombination efficiency to have 100 percent identity between the homology domains of targeting construct and the targeted genomic region. Therefore, it was also decided to prepare the homology domain of the next construct from mouse genomic $\lambda$-phage clones isolated from a $\lambda$-phage library.

In order to generate conditional knockout mutant for unc $5 A, 3$ homology arms of the following sizes: $3.7 \mathrm{~kb} 5^{\prime}-$ homology, $8.4 \mathrm{~kb}$ internal homology and $4.2 \mathrm{~kb} 3^{\prime}-$ homology and LoxP sites were included at appropriate places (Figure 6B). The 
transgenic mouse-line created via this construct would have a CRE-dependent deletion of all the transmembrane and intracellular parts of the unc5A protein. A neomycin cassette and 2 TK cassettes were placed for positive and negative selections of ES clones, respectively. Few number (107) of ES clones were obtained after both selections and none had the desired homologous recombination (Figure 6B). A new construct was prepared in a similar way as the previous one but putting only one TK cassette instead of two, in order to weaken the selective pressures to obtain more ES-cell clones (Figure 6C). Another important reason for decreasing the number of TK cassettes in this construct was the idea that having an identical $2.0 \mathrm{~kb}$ region on both ends of the construct could possibly prevent the linearity of the construct within the ES-cells via the paired alignments between these two DNA sequences.

After the electroporation of this new construct and both positive and negative selections, 161 ES-clones were obtained. None of them had the desired homologous recombination, which should be between the 5'- and $3^{\prime}$-homology regions. However, there were 2 clones that had the homologous recombination between the $5^{\prime}$ - and internal-homology regions. These clones would not allow us to create the loss-offunction mutants, since only one of the LoxP sites were integrated into the locus. However, this result showed us that we could succeed to access the unc $5 \mathrm{~A}$ genomic locus by using these large homology arms, although the recombination efficiency would be still lower than 1\% [2 ES-clones out of 107+161=268 ES clones in total].

When compared to the $5^{\prime}$ and the 3 'homology arms, the conditional knockout construct had a quite big internal homology arm. It seems advisable to keep the sizes of internal homology arms as small as possible in order to increase the chance of homologous recombination event between the $5^{\prime}$ - and the $3^{\prime}$-homology regions instead of between the internal homology and one of the $5^{\prime}$ - and $3^{\prime}$-homology regions (personal communication with Ashery-Padan R.). However, due to the special exonic structures of the unc5A gene, such construct with a large internal homology region was unavoidable. Keeping the internal homology small by targeting only few exons would not disrupt the entire open-reading frame (ORF) of the gene, since almost all exons possess ORFs, which are in frame with both the up- and downstream exons.

Keeping the above observations concerning the homologous recombination efficiency in the unc $5 \mathrm{~A}$ locus in mind, a new conventional construct was generated. This construct contained a total homology of $13.0 \mathrm{~kb}$ and targeted a big portion of the 
gene encoding for the whole transmembrane and intracellular parts and most of the extracellular parts of unc5A protein (Figure 6D). As a reporter, TauLacZ fusion gene was used again to replace the deleted region of unc5A gene. 310 ES clones were obtained with this construct after selections, and 2 of them were identified as positive clones (Figure 6D-E). We had an unusual observation for one of these clones (ES\#264) as the band corresponding to the mutant allele had a much higher intensity for the screen with $5^{\prime}$-internal probe (Figure 6E). After intensive screenings by using different restriction enzymes and hybridization probes, it was realized that this ES-clone had two copies of the targeting construct. One of them was integrated into the correct locus of unc $5 A$ via homologous recombination, whereas the other one in a different wrong locus via non-homologous recombination. Nevertheless, both of these ES-clones were used in order to generate chimaeras.

The same construct was electroporated once more into the ES cells in order to obtain more positive ES-clones. Screening 210 new ES-clones resulted in only one new positive clone (Figure 6D-E). Together from 2 electroporations, 520 ES cell clones were screened producing 3 recombinants, revealing a recombination efficiency of $0.58 \%$. This observation also confirms our initial speculation of low recombination efficiency within the unc $5 A$ genomic locus.

Although gene targeting by homologous recombination in ES-cells is a technique used for quite a long time, the real mechanisms that affects the recombination efficiencies are not known. For example, the effects of the ES-cell state during the electroporation (like the cell-cycle stages) is not known, which is the reason why it is not being controlled during these experiments. On the other hand, it is observed also by many others that different genomic loci have different tendencies for homologous recombination; however, there is no clue about on what this tendency of recombination depends. Therefore, it is impossible to foresee the success rate of a targeting construct.

There are two different techniques to generate chimaeras from ES-cells: Aggregation and blastocyst injection. First, the aggregation method was tried with all three positive ES clones. Four aggregations with ES\#23 clone generated only 3 chimaeras with low chimaerism, which did not give any germline transmission. Two trials of aggregation with ES\#264 gave 6 chimaeras with low chimaerism and no 
germline transmission. For ES\#110, no chimaeras were obtained from two different sets of aggregation.

It has been observed that the chimaeras generated by blastocyst injection method generally have a higher success in germ-line transmission as compared to the ones generated by aggregation (personal communication, Franke U.). Therefore, blastocyst injection method was tried with all three ES-cell clones. ES\#264 did not produce any chimaera. On the other hand, 5 and 12 chimaeras were obtained from two different blastocyst injections of ES\#23, respectively. All these chimaeras were with low chimaerism and did not give any germ-line transmission. However, ES clone \#110 produced 17 chimaeras with high chimaerism (up to 100\%), and 8 of them transmitted the knockout mutation through germline.

It has been observed by us and others that not all positive ES clones from the same targeting experiment generates chimaeras with high chimaerism and their germline transmissibility is also variable. Although the real explanation for such cases is not known, this could be due to some possible spontaneous mutations occurring in some ES-clones during electroporation or culturing. The nature of the type of such mutations that will effect the generation of chimaeras is not known. Apart from such a genetic reason, the probability effect for the random distribution of these chimaeric blastocyst cells is also important. For ES\#23 clone, all the chimaeras produced either with 4 sets of aggregation or 2 sets of blastocyst injection had really low chimaerism. So a genetic explanation sounds more probable than just the matter of a chance.

There are also several technical considerations that affect the success of aggregation or blastocyst injection methods in terms of creating highly chimaeric mice. Preparation of the ES-cell culture in order to be used for both of these techniques is important. For example, they should be kept at low confluence before starting the aggregation or blastocyst injection. The state of the ES-cells (for example, the stage of their cell-cycle) just before being used in these techniques may affect the chimaerism level of the resulting embryos. Preparation of morulas for aggregation and blastocysts for blastocyst injection should be done with extreme care. Small differences during their preparations at different times may give different rates of success for the respective method. These are possibly the explanations for why the aggregations (or blastocyst injections) of the same ES-clone in different times give different results in terms of chimaerism, as observed in this study. 


\section{Analyses of unc $5 A$ loss-of-function phenotypes}

The unc $5 \mathrm{~A}^{-/-}$mice were viable and fertile, did not have any general health problems in mixed 129xCD1 background. In preliminary behavioral analyses, we did not observe any differences compared to their wildtype littermates for olfactory behaviors. Some of the homozygotes, but not all, were observed to be blind according to the simple behavioral tests.

Actually such a visual system phenotype was expected, since unc5A is expressed in the neuroretina (Engelkamp D., 2002) and also in the almost all the brain structures important for the visual system, as explained before. However, the reason why we cannot observe the same phenotype in all homozygotes is not clear. The difference between these different homozygous mice could be due to having a mixed background, since it was also shown that the phenotype for unc $5 \mathrm{C}$ knockout mice is dependent on the actual background of these mice (Burgess RW. et.al., 2006). Homozygous mice for the null mutation in unc5C locus survive the normal life-span in B6xSJL mixed background, although they have numerous CNS phenotypes, like cerebellar hypoplasia, granule and Purkinje cell mismigration and defects in corticospinal tract path-finding. However the same mutation causes perinatal death due to the apparent respiratory distress in pure C57/BL6-J background, because of the incomplete phrenic nerve innervations of the diaphragm muscle (Burgess RW. et.al., 2006).

The loss-of-function of another gene, acetylcholinesterase (AChe), which is believed to function in axonal outgrowth mechanisms during neural development (Bigbee JW. et.al., 1999), also shows different types of phenotypes depending on the backgrounds of the analyzed mice. These mice survive to adulthood in 129 background, whereas they die during seizures before PN-21 in inbred C57/BL6 or outbred CD1 backgrounds (Duysen EG. et.al., 2006).

During the analysis of the adult brains, we also observed that approximately $15 \%$ of the unc $5 \mathrm{~A}^{-/-}$mice had smaller cerebral hemispheres compared to their wildtype littermates. The extent of this size difference was also variable. It is important to know that the cerebral hemispheres of CD1-outbreed mice are usually bigger than the ones for 129-inbred mice. However, the genetic explanation for this observation is not clear. Since the analyzed F1 mice were in completely mixed CD1x129 background, it is 
possible that this 'smaller cerebral hemisphere' phenotype is not due to the loss-offunction of $u n c 5 A$, but the mixed background effect.

Because of encountering such mixed-background problems, it is important to analyze the phenotypes of $u n c 5 A$ knockout mice in the pure inbred background. Among the most commonly used inbred mouse strains, different subgroups of C57/BL6 background mice offer several advantages. First of all, most of our current knowledge about neocortical development and axon guidance systems is based on the studies using the mice of this strain. Interestingly, the expression patterns of some marker genes for neocortical regionalization and the developmental timings of most of the axon-guidance events differ between mice of different backgrounds. Therefore, it is important for our studies to use the similar genetic background, in which most of our current knowledge was produced. Working with C57/BL6 background mice also offers an advantage for general behavioral analyses. No apparent defects for the sensory systems, like visual, olfactory or auditory systems, were detected in this inbred mouse strain. Furthermore, the mice of this background have high reproductive successes and give satisfactory litter sizes (average of 8-10 pups per litter) for comparative littermate analyses.

Because of these advantages of C57/BL6 inbred background, I started to backcross the unc $5 \mathrm{~A}$ knockout mouse line into this inbred background. It is necessary to have at least 7 generations of backcrossing (N7) in order to reach an acceptable congenic state (>99\%). Since these extremely time-consuming backcrossings are still in progress, the phenotypical investigations for the unc $5 \mathrm{~A}$ loss-of-function mutants in this background cannot be started yet.

Germ cells of the successful chimaeras were generated from the ES-cells derived from the pure 129-background embryos. Therefore, it was possible to obtain the unc $5 \mathrm{~A}$ knockout line directly in pure 129 background. In this respect, I started to analyze the unc $5 A$ knockout line in the inbred 129 background, while the backcrossings into C57/BL6 background was in progress.

The unc $5 \mathrm{~A}^{-/-}$mice did not have any general health or reproduction problems in pure 129 background. According to the preliminary behavioral analyses, no differences between homozygote and wildtype littermates were observed for olfactory, auditory or visual behaviors. The previously observed blindness phenotype in CD1x129 mixed background disappeared in pure 129 inbred background. This could be explained by the presence of some unknown genetic traits present in 129-background that rescues the 
blindness phenotype. Therefore, the littermates among the CD1x129 mixed background animals, which did not have these rescuing genetic traits (due to differential segregation of genetic elements), most probably showed such a blindness phenotype; whereas for the ones that had those genetic traits, the phenotype was rescued.

In terms of general morphology of cerebral cortex and other brain regions, no gross abnormalities or significant differences between the wildtype and homozygote littermates were observed. However, some genotype-independent differences in all commissural pathway regions of the forebrain (the corpus callosum, hippocampal commissure, and anterior commissure) were observed. It is known that some inbred mouse backgrounds, like BALB/c and 129, has some abnormalities in all these commissural pathways. Although these inbred lines are genetically highly homogenous, different embryos within the same set of litter coming from WTxWT matings show differences. For example, some has no corpus callosum, whereas others have with varying sizes and areas (Wahlsten D. et.al., 2003; Wahlsten D. et.al., 2006). Therefore, the analyses for identifying a defect in axonal guidance mechanisms of corticogenesis could not be done effectively in this 129-background. However, after obtaining the unc5A knockout line in pure C57/BL6-N background, it will be possible to test all our hypotheses concerning the deficiencies in axon guidance mechanisms of corticogenesis.

Further analyses for identifying a minor neocortical regionalization defect was also not possible in this study because of two reasons. Most importantly, the expression patterns of some of the regional marker genes were showing subtle differences in 129 background compared to the published studies, where they were established as a regionspecific marker for the neocortex of C57/BL6 background mice. Secondly, the small litter size (4-6 pups per litter in average) obtained in 129 background was mostly preventing any 'within-litter' analyses, since the probability of getting both homozygous and wildtype embryos or pups is lower in such a small litter size. However, in future, it will be possible to test all our hypotheses concerning the functional role of unc $5 \mathrm{~A}$ in neocortical regionalization when the unc $5 \mathrm{~A}$ knockout line will be obtained in C57/BL6-N background because of all the advantages of this background as mentioned earlier.

The generation of $u n c 5 \mathrm{~A}$ loss-of-function mutants and their phenotypical analyses during spinal cord development was very recently published by others (Williams ME. 
et.al., 2006). In that study, they generated the unc5A knockout line by targeting the genomic regions between the $4^{\text {th }}$ and $8^{\text {th }}$ exons, which encodes the most of the extracellular part and transmembrane domain of unc5A protein. They also tried to replace these targeted regions of unc5A with an 'IRES-TauLacZ' cassette. They observed a reduction in the apoptosis within the motor neuron columns of E14 cervical spinal cord, which resulted in having more of Islet $1 / 2$ positive cells. Interestingly, this phenotype do not cause any observed abnormality in the motor activity of these homozygous mice. Within this study, they do not mention in which kind of mouse background, they observed this phenotype.

Surprisingly, they do not show any data obtained by LacZ staining with the knockin TauLacZ reporter. In our studies, we also did not obtain any kind of LacZ staining patterns for neither embryos nor adult brains. Both our and their targeting strategies were similarly replacing the $u n c 5 \mathrm{~A}$ sequences downstream of $4^{\text {th }}$ exon with a TauLacZ reporter gene. The expressions of TauLacZ gene were shown at RNA level for both heterozygous and homozygous animals (Figure 7B). However, a functional TauLacZ activity was obviously not produced by these transcripts. This could be due to the suppression of its translation by unknown post-transcriptional or translational regulation mechanisms.

\section{Analysis of unc5A gain-of-function mutants}

A different genetic approach widely used to understand the function of genes is to analyze the phenotypes of gain-of-function mutants for those genes. This approach also gives the scientists deeper insights for understanding many diseases, in which a mutation causes the misexpression of a gene.

With ectopic overexpression of $u n c 5 A$, which encodes a chemorepulsive netrin receptor, the significance of Netrin-dependent axon guidance mechanism can be elucidated in two different ways: (1) By ectopically overexpressing unc $5 \mathrm{~A}$ in the regions where netrin-dependent chemoattraction is crucial for guiding the axons (or directing the cellular migrations), this chemoattractive mechanisms could be disrupted or converted to chemorepulsion. The observed phenotypes would indicate the direct or indirect functional importance of these disrupted mechanisms during the development. 
(2) During axonal pathfinding, neurons switch on and off the expression of several axon-guidance receptors in order to respond differently for the same type of axon guidance ligands along the distinct regions of their path. By constitutively overexpressing unc5 $\mathrm{A}$ in the axons where it is already being expressed with a temporal regulation, the importance of the switch mechanisms could be understood for the corresponding axonal path-finding processes.

Therefore, transgenic lines for conditionally overexpressing unc $5 \mathrm{~A}$ were created with the aim of characterizing the functional role of $u n c 5 A$ and the Netrin-dependent axon guidance mechanisms during neocortical development. Since the " $\Delta$ exon6" spliced variant is the major form expressed during embryogenesis (Figure 5), I decided to overexpress this variant of $u n c 5 A$ instead of the full-length form. The functional difference between these two forms of $u n c 5 A$ is not known. The $6^{\text {th }}$ exon of $u n c 5 A$ gene encodes for one of the two TSP domains within the extracellular part of the unc5A protein. These TSP domains of UNC5 family of proteins could be important either for interacting with different ligands and other extracellular or cell-adhesion molecules in the surrounding or just for the stability of the conformational structure of their extracellular parts. It was shown, at least in vitro, that IG domains of UNC5 family of proteins are the responsible ones for binding to the Netrin ligands (Geisbrecht BV. et.al., 2003).

By using the transgenic mouse lines generated with this conditional overexpression construct (Figure 9), it would be possible to decide when and where to overexpress unc $5 A$ depending on the CRE-line that will be used.

Three founder lines were generated by using this construct (Figure 9). It is important to have more than one founder lines while working with transgenic mice in order to rule out the possibility that any observed phenotype in the experiments are actually coming from the disruption of a certain genomic locus during the integration of the transgenic construct. Since the genomic integration site of the construct is random, it is unlikely that two completely different founder lines will have the same genomic site for the integration of the transgenic construct. Therefore, observing same phenotype by using more than one founder line ensures the reliability of the obtained results.

Seven of the 323 generated mice by four trials of microinjection of the same construct were genotypically positive. However, 1 of these 7 founder lines did not give any progeny, whereas 3 of them gave progeny without uniform expression of eGFP. 
The other 3 founder lines were giving progenies with expected uniform eGFP expression both in embryonic and adult stages (Figure 9C). In generating transgenic mouse lines, it is well known that not all genotypically positive founder lines will give the expected expression patterns. This is most probably due to the silencing of the transgene's expression by the genomic elements surrounding the random integration site of the constructs.

The uniform eGFP expression in all the embryonic and adult stages of each 3 generated founder lines showed that the strong composit CMV-IE and B-actin promoter is functioning well for the overexpression (Figure 9C).

\section{Ectopic overexpression of unc5A-Aexon6 in the embryonic distal neuroretina} causes blindness in a background-dependent manner

In all neural sensory systems, the correct spatial representation of the neural inputs within the corresponding thalamic and neocortical regions are important. For example, the spatial representation of the sensory neurons of the nasal olfactory membrane is established as 'glomeruli' of olfactory bulbs, and then as 'septal nuclei' in MOA. Another well-understood example is the perfect spatial representation of whiskers in the 'barrellettes' of thalamus, and then the 'barrels' of somatosensory cortex. These perfect spatial representations could be important for the development of neocortex either in structural (as proposed by 'protocortex hypothesis') or in functional terms. Therefore, we aimed to genetically disrupt the mechanisms responsible for the spatial representation of neuroretina in the corresponding thalamic nuclei and visual cortex in order to see its effect in the structural and functional development of visual cortex.

It was shown that unc5A is expressed within the outer layers of the central neuroretina as early as E12.5 (Engelkamp D., 2002). In contrast, the chemoattractive receptor, $d c c$, is expressed in the innermost layers of the central neuroretina; and another chemorepulsive receptor, $u n c 5 B$, is expressed in the dorsal half of the neuroretina (Engelkamp D., 2002). These differential expressions of the three Netrin receptors suggest the presence of a specific Netrin-dependent axon guidance mechanism for the correct spatial representation of the neural inputs brought by the optic nerve. 
Therefore, we postulated that the ectopic overexpression of unc5A in the distal part of neuroretina would either cause the misrepresentation of this spatial order or block the representation of distal neuroretina in optic nerve completely. With this purpose, the ECG-Cre line, which expresses CRE protein under the control of Pax6 gene's neuroretinal enhancer element, was used. This transgenic line gives CRE activity in all the retinal progenitor cells within the distal neuroretina very early during the development of neuroretina (Figure 10 and Marquardt T. et al., 2001).

Blindness phenotype was checked for generated double transgenic animals. Surprisingly, a background-dependence was observed for this phenotype. Some of the wildtype and single transgenic littermates were also blind as some of the double transgenic littermates. All the analyzed hybrid animals were in a mixed background for 3 different backgrounds: FVB, C57/BL6-N, and B6CBA. It is known that FVB- inbred background is homozygous for the retinal degeneration allele $P d e 6 b^{r d l}$ (JAX mice Data Sheet for FVB/NJ. The Jackson Laboratory; JAX Notes. Spring 2002). It was also announced that B6CBA inbred strain has mutations at the Mitf locus that affects the eye size and pigmentation. (JAX mice Data Sheet for B6CBA. The Jackson Laboratory). Therefore, independent of the unc $5 A$ overexpression, some of the hybrids were showing the blindness phenotype as a result for the combination of genetic traits that were segregated from each of these 3 different inbred backgrounds. As expected, the blindness phenotype, which is independent of unc5A overexpression, is mostly diminished when the partially backcrossed ECG-Cre line (from FVB background into the C57/BL6-N background for 3 generations) was used. Since these hybrids had less contribution of FVB background, it is clear that this 'unc5A-overexpressionindependent' blindness was due to the genetic traits of FVB background.

On the other hand, the ratio of double transgenics that gave blindness phenotype was increasing with every backcrossing generation of unc5A-overexpression line. Obviously, this increase was in parallel with the decrease of the contribution of B6CBA background, since the transgenic founders were generated in B6CBA background and then backcrossed into C57/BL6-N background. This observation suggests that some genetic traits present in B6CBA inbred-background actually rescue the blindness phenotype of some of the double transgenic animals.

Different E12.5 embryos, which overexpress unc5A in distal neuroretina, showed different patterns of LacZ stainings (Figure 11). Since we could not know which one of 
these embryos would reflect the situation for the blind hybrids, it is impossible to explain the reason of blindness. However, one important observation for these embryos is the lack of staining from the ventral part of the distal neuroretina moving toward the central neuroretina at the exit point for the optic nerve (compare Figure $11 \mathrm{~A}, \mathrm{C}$, and D with Figure $10 \mathrm{E}-\mathrm{F})$. This could suggest that the axons that should represent the distal neuroretina are not able to reach the optic nerve. However, these observations should be confirmed later by more extensive molecular analyses.

According to all these observations concerning the above mentioned "background effects" in blindness, it is obviously important to backcross all the used transgenic lines into the pure C57/BL6-N background prior to any phenotypical investigations. By this way, the reproducible and significant results concerning the blindness phenotype could be obtained, and its effect on neocortical development can be investigated. However, due to the extremely long time span needed to backcross the mice (over 1 year), these experiments could not be performed within the context of this study.

\section{Ectopic overexpression of unc5A-Dexon6 in neocortex and hippocampus}

I then decided to use the Nex-Cre transgenic line in order to overexpress unc $5 \mathrm{~A}$ in the post-mitotic neurons of all neocortical regions and also in the hippocampal pyramidal neurons. This could allow us to test 3 different hypotheses concerning the importance of Netrin-dependent axon guidance systems during the structural and functional development of neocortex and hippocampus: (1) The neocortical regions, where the Netrin-dependent chemoattraction mechanism is important, could be identified by ectopically overexpressing $u n c 5 A$ in those regions. Since it is assumed that unc $5 A$ normally should not be expressed in those regions, such an overexpression would prevent or impair the axonal connections of the neurons belonging to those neocortical regions. (2) The 'Switch on/off' mechanism ensures that during the axonal path-finding processes, the axons will respond to the same axon guidance cues in different manners along their paths by spatio-temporal regulation of the corresponding attractive or repulsive receptors' expressions. By forcing a continuous overexpression of $u n c 5 \mathrm{~A}$ in the neurons where it is already being expressed, the significance of these 
'switch on/off' mechanisms for the axonal path-finding of several systems could be understood. (3) Learning and memory functions of the brain are regulated by the hippocampus via its connections with the different neocortical regions. By overexpressing the unc $5 \mathrm{~A}$, some of these axonal connections that require the Netrindependent chemoattraction mechanism could be identified, since they will be disrupted by the chemorepulsive activity of $u n c 5 A$.

TauLacZ reporter that is supposed to be expressed together with the overexpression of $u n c 5 \mathrm{~A}$ would be useful for analyzing these postulated axonal defects. Unfortunately, no LacZ staining by this reporter was detectable by any CRE-lines used in this study. However, the CRE-activity dependent expression of TauLacZ was shown at RNA level (Figure 13) and in vitro by using Neuro2A cell-lines (Figure 14). Although the reasons for this absence of LacZ activity is not clear, it could be due to some unknown post-transcriptional or translational regulation mechanisms that suppress its expression at protein level.

Generated hybrid animals for the overexpression of unc $5 \mathrm{~A}$ in the Nex expressing regions, were healthy and fertile. The detailed histological and behavioral analyses for testing our hypotheses have still to be done in future. 


\section{MATERIALS AND METHODS}

\section{$\underline{\text { Organisms }}$}

\section{$\rightarrow \underline{\text { Mouse }}$}

NMRI and CD1 wildtype outbred strains of mice were used for the ISH-screen of selected genes from microarray dataset as well as RACE and RT-PCR analyses for the detection of $u n c 5 A$ spliced variants and expression kinetics.

The mouse knockout line for unc5A gene was produced by using 'MPI-II' ES cells of $129 / \mathrm{Sv}$ origin. CD1-outbreed strain mice were used during the germline screenings. 129/Sv and C57/BL6-N inbred strains were used during the matings for backcrossings and colony maintenance.

The transgenic lines for the conditional overexpression of $u n c 5 \mathrm{~A}$ were produced in B6CBA background. C57/BL6-N inbred strains were used during the backcrossings.

The transgenic CRE-lines, 'ECG-Cre' (Marquardt T. et al., 2001) and 'Nex-Cre' (Goebbels S. et.al., 2006), and the reporter line, 'Floxed-ROSA' (Mao X. et.al., 1999), were used from the animal stock of the department. FVB and C57/BL6-N inbred strains were used during the matings for backcrossings or colony maintenance.

For overnight matings, the morning when a vaginal plug was observed considered as E0.5. For timed-matings, the time of vaginal plug observation was accepted as E0.

Mice were always sacrificed for analyses by cervical dislocations.

\section{$\rightarrow \underline{\text { Cell-Line }}$}

'Neuro2A' cell line from the cell-line collection of the department was used in the cell-culture studies. 


\section{$\doteq \underline{\text { Bacteria }}$}

The E. coli strain 'DH5 $\alpha$ ' was used for all molecular cloning studies.

Special E.coli strains that express CRE or FLIP recombinase proteins in a temperature-sensitive manner were used for checking the prepared loss-of and gain-of function constructs. These bacterial strains were obtained from departmental stocks of bacteria.

XL1-BlueMRA strains of E.coli (Stratagene) were used for the screening of mouse genomic $\lambda$-phage library.

\section{$\underline{\text { Materials }}$}

\section{$\rightarrow \underline{\text { General laboratory equipments }}$}

All PCR reactions were done in PTC-200 Peltier Thermal Cycler (MJ Research).

Centrifugations in room temperature for small volumes $(>1.5 \mathrm{ml})$ were done on bench-top centrifuges (Heraeus Instruments). For big volumes, RC-50 centrifuges (Sorvall) were used with HS-4 rotors.

\section{$\zeta \underline{P C R}$ primers}

All PCR primers were ordered from IBA-GmbH, Goettingen.

\section{$\rightarrow \underline{\text { Chemicals and enzymes }}$}

All chemicals used for the preparation of experimental solutions were obtained from the suppliers: Merck, Sigma, Roth, Gibco, and Fluka.

All the enzymes for molecular biology experiments were ordered from the companies: Promega, NewEngland Biolabs (NEB), Stratagene, Boehringer-Roche and Invitrogen. The enzymatic reactions with these enzymes were done according to the protocols supplied by the manufacturers.

Taq-Polymerase and other PCR reagents were ordered from GeneCraft.

Other used reagents or kits are mentioned below during the description of the methods. 


\section{$\uparrow \underline{\text { Standard solutions }}$}

All standard solutions like LB, LB-Agar, NZY-medium, PBS, PFA, SSC, TBE, and TE solutions were prepared according to the protocols in Sambrook J. and Russell DW. (2001).

\section{Basic molecular biology techniques}

All plasmid DNA extractions were done with 'QIAprep Miniprep' or 'Plasmid Purification' kit of QIAGEN according to the manufacturer's protocols.

PCR purifications and extraction of DNA fragments from Agarose gels were done with 'QIAquick Spin' kit of QIAGEN according to the supplied protocols.

Radioactively-labeled DNA fragments, which were used as probes for plaque-lifthybridization, colony-lift-hybridization, and southern-blot-hybridization analyses, were prepared from 50-100 ng of specified DNA templates by 'Rediprime II' kit (Amersham) and $50 \mu \mathrm{Ci}$ of ${ }^{32} \mathrm{P}$-dCTP (Amersham) according to the protocol supplied by the manufacturer. Afterwards, the probes were purified via G 50-Spin columns (Amersham) and denatured for 5 minutes at $95^{\circ} \mathrm{C}$ before hybridizations.

All sequencings were done in the sequencing facility (Applied Biosystems, ABIPrism, 3100-AVANT genetic analyzer) of the department.

The protocols in Sambrook J. and Russell DW. (2001) were used for:

- Preparation of plasmid DNA by Alkaline Lysis method

- Agarose Gel electrophoresis

- Determinations of the concentrations for nucleic acids

- Phenol-Chloroform purification of DNA

- Ethanol precipitation of DNA samples

- Preparation of the electrocompetent E.coli bacteria

- Transformation of E.coli by electroporation 


\section{$\underline{\text { In silico gene-prediction studies and Bioinformatics }}$}

For the unknown genes (ESTs) within the E14.5 microarray dataset, in silico gene-prediction studies were done in two steps: Initially, the sequences of the microarray probes for the corresponding gene was used to identify all the EST sequences deposited in the GenBank database of NCBI by using BLAST search program of NCBI (http://www.ncbi.nlm.nih.gov/BLAST/). The consensus sequences from these alignments were derived from at least three independent sets of ESTs. These consensus sequences were again used for a further BLAST alignment with the GenBank database to expand the obtained putative cDNA sequences with the same selection criteria. This process was repeated till it did not result in any further expansion. In the second step, these putative cDNA sequences were first in silico translated into protein sequences. These peptide sequences were then used to align with the protein sequence database of GenBank in order to find similarities with known proteins. Subsequently, the same set of protein sequences were used to determine the putative protein motifs by PROSITE program of Expasy database (http://www.expasy.ch/prosite/).

Exon-intron boundaries for the selected genes were determined by aligning their predicted cDNA sequences with the mouse genomic DNA databases from CELERA (http://www.celera.com/), Ensembl (http://www.ensembl.org/), and NCBI (http://www.ncbi.nlm.nih.gov/).

\section{In situ hybridization (ISH) analyses}

For the in situ hybridization analyses of the selected genes from the microarray dataset, the EST clones of Image Consortium (RZPD, Berlin) were purchased in order to use them for the RNA probe preparations (Table 1). After sequence verifications, the plasmid DNA samples for each were prepared by 'Plasmid Purification' kit (QIAGEN)

\section{I) Synthesis and purification of dig-labeled RNA probes}

Plasmids containing corresponding EST clones were linearized with appropriate restriction enzymes and used as templates for the in vitro transcription reactions using corresponding RNA Polymerase enzymes (Table 1). 
Table 1: The EST clones used as templates for the preparation of ISH probes. Gene nomenclatures are the names given during this study. GenBank Accession Numbers are given for each EST clone. Restriction enzymes used for their linearizations and the necessary RNA polymerases for corresponding antisense RNA probe production are shown.

\begin{tabular}{cccc}
$\begin{array}{c}\text { Gene } \\
\text { Nomenclature Acc.No }\end{array}$ & $\begin{array}{l}\text { Restriction } \\
\text { Enzyme }\end{array}$ & $\begin{array}{l}\text { RNA } \\
\text { Polymer } \\
\text { ase }\end{array}$ \\
\hline AU-1 & BG921984 & SalI & T7 \\
AU-2 & AW210387 & SmaI & SP6 \\
AU-3 & AA219849 & XhoI & T3 \\
AU-4 & AI588076 & SmaI & T7 \\
AU-5 & AI503811 & NotI & T7 \\
AU-6 & BF018619 & KpnI & T3 \\
AU-7 & AI391420 & XhoI & T3 \\
AU-8 & BF135045 & SalI & T7 \\
AU-9 & BF783046 & SmaI & T7 \\
AU-10 & AA038241 & XhoI & T3 \\
AU-11 & AA423063 & XhoI & T3 \\
AU-12 & AI788815 & XhoI & T7 \\
AU-13 & AW412254 & SalI & T7 \\
AU-14 & AA123140 & SmaI & T7 \\
AU-15 & BI686758 & SalI & T7 \\
AU-16 & AA218393 & XhoI & T3 \\
AU-17 & BE449028 & XhoI & T3 \\
AU-18 & BG298307 & SalI & T7 \\
AU-19 & AW260073 & XhoI & SP6 \\
AU-20 & AI323029 & XhoI & T3 \\
AU-21 & BI556086 & SalI & T7
\end{tabular}

$1 \mu \mathrm{g}$ of purified linear EST clones were used for $20 \mu 1$ of in vitro transcription reactions at $37^{\circ} \mathrm{C}$ for 2 hours according to the manufacturer's protocol ('DIG RNA Labeling Kit' by ROCHE).

Synthesized RNA probes were diluted into a final volume of $100 \mu$ with TE $(\mathrm{pH}$ 8.0) and precipitated with $10 \mu \mathrm{l}$ of $4 \mathrm{M} \mathrm{LiCl}$ and $300 \mu \mathrm{l}$ of Ethanol at $-80^{\circ} \mathrm{C}$ for 2 hours. After centrifugations at $20.000 \mathrm{xg}$, the pellets were washed with $100 \mu 1$ of $70 \%$ Ethanol and final RNA pellets were dissolved in $100 \mu \mathrm{l}$ of TE ( $\mathrm{pH} 8.0)$. Integrities and the concentrations of each RNA probe were checked on Agarose gels (1\%). Probes were stored at $-20^{\circ} \mathrm{C}$. 


\section{$\rightarrow$ Preparation of embryonic mouse brain sections}

NMRI-outbreed strain mice were mated overnight. After sacrificing the pregnant female mice at days E14.5 and E18.5, embryos were collected and embryonic brains were dissected under a dissection microscope. The isolated brains were immediately put in to $4 \%$ PFA solution and fixed at $+4^{\circ} \mathrm{C}$ for 6 to 8 hours. After three times of $1 \mathrm{X}$ PBS wash for 20 minutes each, they are cryoprotected in 30\% Sucrose solution (in PBS) at $+4^{\circ} \mathrm{C}$. They were embedded in 'Tissue Freezing Medium' (from 'Jung') on dry ice after they started to sink within the $30 \%$ sucrose solution.

The tissue blocks were sectioned for $16 \mu \mathrm{m}$ coronal sections with the cryostat (Leica), and sections were taken onto 'SuperFrost Plus' slides (Menzel-Glaser). After the preparation of all sections, the slides were stored at $-20^{\circ} \mathrm{C}$ for at most a week till performing the ISH analyses.

\section{$\rightarrow \underline{\text { Non-radioactive } R N A \text {-in situ hybridization }}$}

All incubations are in room temperature (RT) unless it is mentioned otherwise.

After partially thawing the sections, they were fixed in 4\% PFA for 15 minutes and then washed twice with $1 \mathrm{X}$ PBS for 5 minutes each. The proteolytic digestions were done with Proteinase $\mathrm{K}(20 \mu \mathrm{g} / \mathrm{ml})$ at $37^{\circ} \mathrm{C}$ for 4 minutes. This permeabilization of sections were stopped with $0.2 \%$ Glycine (in PBS) and then they are washed twice with 1X PBS for 5 minutes each. Afterwards, they were post-fixed with $0.2 \%$ Glutaraldehyde / 4\% PFA (in PBS) for 20 minutes. They were again washed twice with 1X PBS for 5 minutes each, and then prehybridization solution was put onto them. After the 2 hours of incubation at $70^{\circ} \mathrm{C}$, the prehybridization solutions were replaced with hybridization solutions (prehybridization solution together with the denatured DIGlabeled RNA probes). Hybridizations were done overnight at $70^{\circ} \mathrm{C}$.

Next day, the hybridization solutions were discarded, and sections were washed three times with $2 \mathrm{X} \mathrm{SSC}(\mathrm{pH} 4.5) / 50 \%$ Formamide solutions at $65^{\circ} \mathrm{C}$ for 30 minutes each. Afterwards, they were washed twice with KTBT solution for 10 minutes each, and then blocking solution was put onto them. After the 2 hours of incubation, the blocking solutions were replaced with the antibody solutions (1:2000 anti-DIG antibody in blocking solution) for an overnight incubation at $+4^{\circ} \mathrm{C}$. 
On the third day, the antibody solutions were discarded, and the sections were washed with KTBT solution for three times of 5 minutes each and a further 3 times of 30 minutes each. Afterwards, they are washed three times with NTMT solution for 5 minutes each. The color development reactions were started by adding the NBT-BCIP solution (from Roche) in NTMT (1:50) onto the sections. After obtaining satisfactory staining, the color development reactions were stopped by three times washing with $1 \mathrm{X}$ PBS for 5 minutes each. Then the slides were mounted with Mowiol, and the pictures were taken under a light microscope with a digital camera (SZX-12 Olympus).

For all the ISH analyses done, proper controls with 'sense' probes were done. No signals for any of these 'sense' groups were observed.

\section{¿) $\underline{\text { Solutions used in ISH }}$}

All solutions used in the first day of ISH were prepared by DEPC (SIGMA)treated solutions in order to prevent the RNase contamination.

Prehybridization solution (final concentrations of the ingredients):

$\begin{array}{ll}50 \% & \text { Formamide } \\ 5 \mathrm{X} & \text { SSC } \\ 1 \mathrm{mg} / \mathrm{ml} & \text { Yeast tRNA } \\ 0.1 \%(\mathrm{w} / \mathrm{v}) & \text { CHAPS } \\ 0.1 \%(\mathrm{v} / \mathrm{v}) & \text { Tween-20 } \\ 5 \mathrm{mM} & \text { EDTA }(\mathrm{pH} 8.0) \\ 0.1 \mathrm{mg} / \mathrm{ml} & \text { Heparin }\end{array}$

KTBT solution (final concentrations of the ingredients) :

$\begin{array}{ll}50 \mathrm{mM} & \text { Tris-Cl }(\mathrm{pH} 7.5) \\ 150 \mathrm{mM} & \mathrm{NaCl} \\ 10 \mathrm{mM} & \mathrm{KCl} \\ 1 \%(\mathrm{v} / \mathrm{v}) & \text { Triton-X-100 }\end{array}$

NTMT solution (final concentrations of the ingredients) :

$\begin{array}{ll}100 \mathrm{mM} & \text { Tris-Cl (pH 9.5) } \\ 100 \mathrm{mM} & \mathrm{NaCl} \\ 50 \mathrm{mM} & \mathrm{MgCl}_{2} \\ 0.05 \%(\mathrm{v} / \mathrm{v}) & \text { Tween-20 }\end{array}$




\section{$\underline{\text { RACE analyses for AU-18 (unc5A) }}$}

In order to determine the $5^{\prime}$ - and $3^{\prime}$-ends of the cDNA for unc5A, 5'-and $3^{\prime}-$ RACE (Rapid Amplification of cDNA Ends) analyses were done by using the total RNA isolated from the pooled E18.5 brain samples.

\section{$\zeta \underline{\text { Total-RNA isolation }}$}

Male and female mice of NMRI-outbreed strain were used in overnight matings. The pregnant female mice were sacrificed at day E18.5. Brains were dissected from the collected embryos under a dissection microscope and immediately frozen on dry ice.

Total RNA was isolated from these pooled brain samples with TRIzol reagent (Invitrogen) according to the manufacturer's protocol. Integrities of the isolated RNA samples were checked on Agarose gels (1.5\%), and the concentrations were determined by the spectrophotometer measurements.

\section{$\Rightarrow \underline{3^{\prime}-R A C E}$ Analysis}

$3^{\prime}$-end of the unc5A cDNA is determined by using the isolated E18.5 brain total RNA and two nested PCR primers for unc5A gene by using the 'GeneRacer Kit' (Invitrogen) according to the protocol supplied by the manufacturer. The unc $5 \mathrm{~A}$ primers were both in forward orientations designed from the $14^{\text {th }}$ and $15^{\text {th }}$ exons' sequences of the predicted cDNA (Au18-3Re14 and Au18-3Re15, respectively):

\section{Au18-3Re14 : 5' - CAGTATCTGCACTGCACCTTCAC -3’ Au18-3Re15 : 5’' GAAATGCTGGCTCTGGAGAGTG -3’}

At the end of the two-step nested-PCR amplifications, a PCR fragment of 1450 bps was obtained. This size was compatible to the predicted unc5A cDNA sequence. This PCR product was purified with 'Qiaquick Spin' kit (Qiagen) and ligated into pGEM-Teasy vector (Promega). $1 \mu \mathrm{l}$ of the ligation mix was electroporated into E.coli. 20 different positive bacterial colonies were randomly picked and analyzed via sequencings. 


\section{$\Rightarrow \underline{5^{\prime}-R A C E}$ Analysis}

The same total RNA sample above is used to determine the $5^{\prime}$-end of the unc $5 \mathrm{~A}$ cDNA also by using the same 'GeneRacer Kit' (Invitrogen). 3 reverse primers were designed from the predicted unc5A cDNA sequences for the 4th exon (Au18-5R1) and $3^{\text {rd }}$ exons (Au18-5R2 and Au18-5R3):

\section{Au18-5R1 : 5'- GACAAGgTAGCACAATGCCTTGCTC -3' Au18-5R2 : 5’ GTAGGCCTTCTGGCTTTTGGTG -3’ Au18-5R3 : 5'- GTACTCCTCCAGCCCAAACACTTTC -3'}

Au18-5R1 was used as gene-specific primer for the 'reverse transcription', and Au18-5R2 and Au18-5R3 were used for the first and second steps of the nested-PCR amplifications, respectively. A PCR fragment of $620 \mathrm{bps}$ was obtained and this size was compatible to the predicted unc $5 A$ cDNA sequence. This DNA fragment was cloned into the pGEM-Teasy vector and 20 randomly picked positive clones were analyzed by sequencing.

\section{$\underline{\text { RT-PCR analyses for determining unc5A spliced variants and }}$}

\section{expression kinetics}

Different stage embryos between E8.5 and E18.5 were collected from overnightmated mice (CD1-outbreed strain) and pooled. Total RNA samples for each sample set were isolated with TRIzol Reagent in the same way as mentioned before.

cDNA samples were synthesized from all isolated total-RNA samples by using 'random hexamers' with the 'First-Strand cDNA Synthesis' kit (Amersham) according to the supplier's protocol.

\section{$\rightarrow$ PCR analyses for identifying the spliced variants}

9 PCR-primers specific for different exons were designed (Figure 16A). All the synthesized cDNA samples above were tested by different combinations of these 
primers with the mentioned PCR-conditions in 35-40 cycles (Table 2). The sequences of the used primers were:

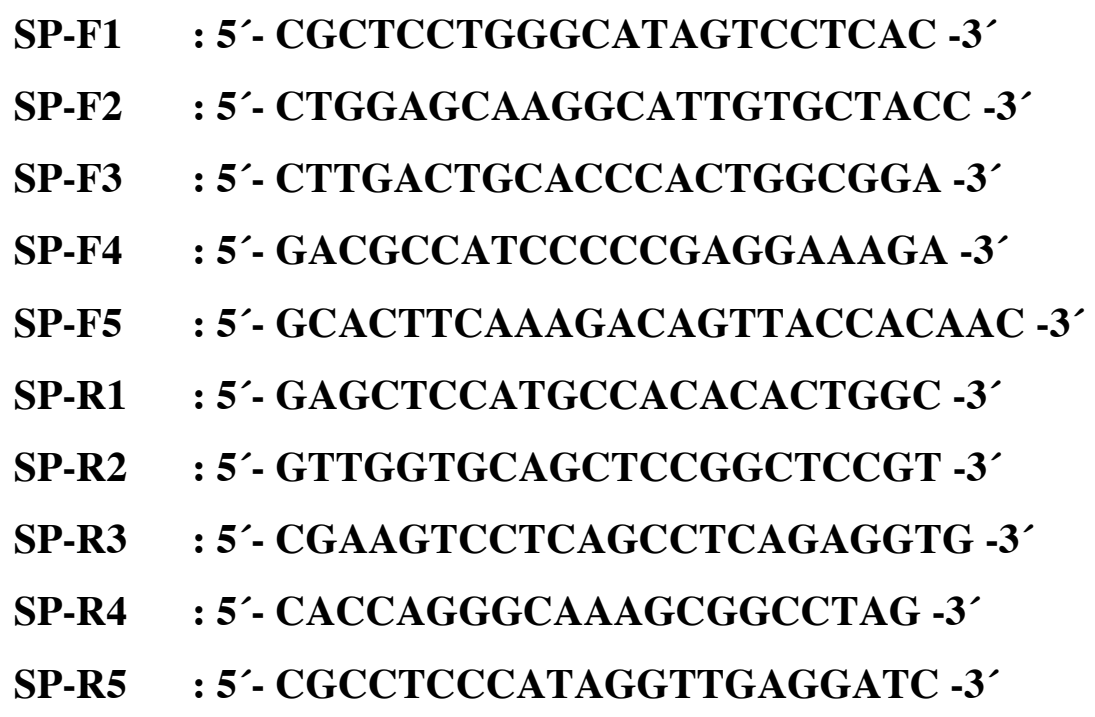

Expected sizes for each PCR reaction according to the full-length unc5A cDNA sequence are mentioned in Table 2. Any specific PCR band with a different size was cloned into pGEM-Teasy vector and sequenced.

\section{$\rightarrow \underline{P C R}$ analyses for identifying the expression kinetics}

A PCR-primer pair was designed by using the sequences in the exonic junctions (Figure 16B), which will determine the full-length and the spliced ' $\Delta$ exon6' variants by the same PCR reaction:

\section{AU-EP3 : 5' - CCAGCTGAGgTGGAGTGGCTC - $3^{\prime}$ \\ AU-EP4 : 5'- GAAGAGgTGTGCAGgCAGAGGTC -3'}

All the synthesized cDNA samples mentioned above were used for the PCR reactions with this primer pair (PCR conditions are mentioned in Table 2). They were also amplified in parallel by PCR with GAPDH-specific primers mentioned below in order to normalize the data for the cDNA concentrations. PCR conditions are mentioned in Table 2.

GAPDH_Frw : 5'-CCGGTGCTGAGTATGTCGTGGAGTCTAC -3' GAPDH_Rev : 5'- CTTTCCAGAGGGGCCATCCACAGTCTTC -3'

cDNA syntheses and all PCR reactions were repeated three times, and the same results were obtained in each set of experiments. 
Table 2. PCR-conditions used for identification of $\boldsymbol{u n c 5 A}$ spliced variants and expression kinetics.

\begin{tabular}{lccccc} 
PCR & $\begin{array}{l}\text { Forward } \\
\text { primer }\end{array}$ & $\begin{array}{l}\text { Reverse } \\
\text { Primer }\end{array}$ & $\begin{array}{l}\text { Annealing } \\
\text { temp }\end{array}$ & $\begin{array}{l}\text { Extension } \\
\text { Time } \\
\text { (seconds) }\end{array}$ & $\begin{array}{l}\text { Expected } \\
\text { sizes }\end{array}$ \\
\hline SP-F1R1 & SP-F1 & SP-R1 & 68 & 30 & $364 \mathrm{bps}$ \\
SP-F2R2 & SP-F2 & SP-R2 & 70 & 30 & $330 \mathrm{bps}$ \\
SP-F3R3 & SP-F3 & SP-R3 & 68 & 30 & $502 \mathrm{bps}$ \\
SP-F4R4 & SP-F4 & SP-R4 & 68 & 30 & $342 \mathrm{bps}$ \\
SP-F5R5 & SP-F5 & SP-R5 & 70 & 30 & $500 \mathrm{bps}$ \\
SP-F1R3 & SP-F1 & SP-R3 & 68 & 90 & $1407 \mathrm{bps}$ \\
SP-F3R5 & SP-F3 & SP-R5 & 68 & 90 & $1662 \mathrm{bps}$ \\
& & & & & \\
EP & EP-3 & EP-4 & 68 & 30 & $530 \mathrm{bps}$ \\
GAPDH & GAPDH_Frw GAPDH_Rev & 72 & 30 & $286 \mathrm{bps}$
\end{tabular}

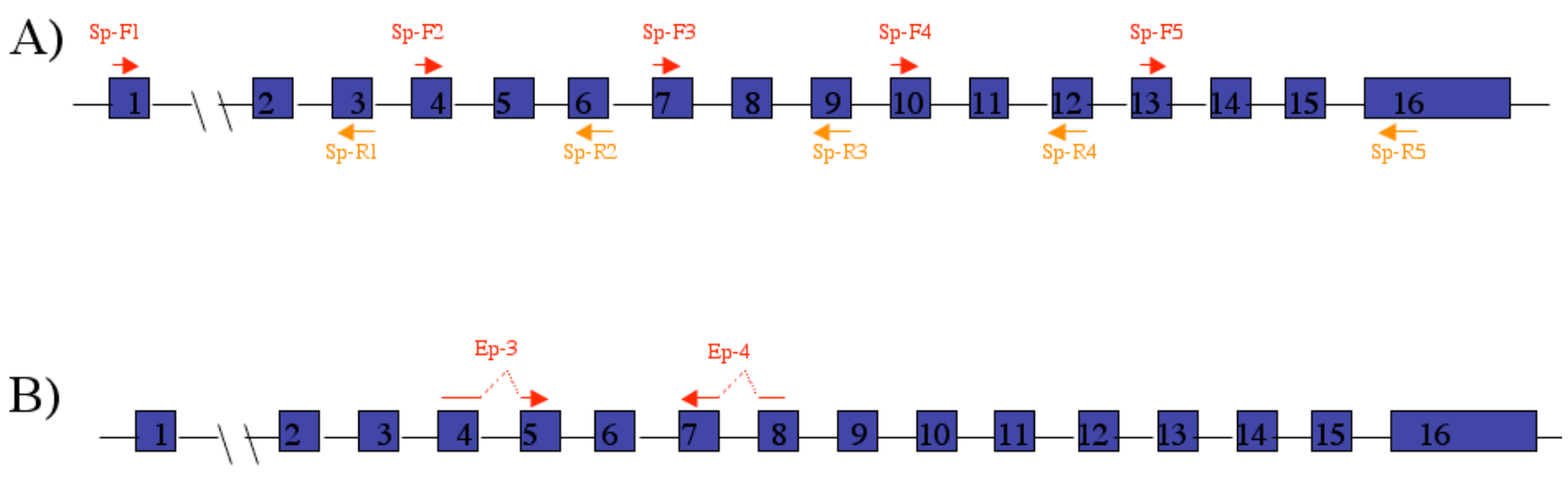

Figure 16. Locations of the PCR-primers used for the identification of unc5A spliced variants (A) and expression kinetics (B). Blue boxes represent the exons of unc $5 A$, whereas the lines between them represent the introns.

A: Forward primers were shown as red arrows above the scheme of gene, whereas reverse primers were shown as orange arrows under the scheme. B: These primers were designed from the junction points of exon 4-to-5 and exon 7-to-8. 


\section{Generation of knockout constructs}

\section{$\neg \underline{\text { First knockout construct }}$}

The first knockout construct (Figure 6A) was prepared for generating the conventional unc5A loss-of-function mutants. Both $5^{\prime}$ and $3^{\prime}$ homology arms were produced by PCR from isolated genomic DNA of ES-cells with following primers:

\section{5'hom_Frw : 5' - GCAACCCTAATTGAATTTCTCTGT -3' \\ 5'hom_Rev : 5'- GTGACCATTAGAGAGCTGGAACTT -3' \\ 3'hom_Frw : 5'- TGATCATTTTCTCAAACCTGAATG -3' \\ 3’hom_Rev : 5’-AGTTGGGAGTTAGTCTAGGTGGTG -3’}

PCR-production of $5^{\prime}$ and $3^{\prime}$ homology arms were done with annealing temperatures of $63^{\circ} \mathrm{C}$ and $61^{\circ} \mathrm{C}$, respectively, and extensions of 5 minutes in 30 cycles. 'Expand Long Template PCR system' (Roche) was used in order to reduce the mutation rate due to the PCR amplification. After each PCR reaction, a single band of approximately $5.0 \mathrm{~kb}$ (as expected) was obtained and then cloned into pGEM-Teasy vector. These homology arms were extensively sequenced from both sides with 30 different internal primers (15 forward and 15 reverse primers) in an overlapping manner in order to see if there are important mutations created by PCR reactions. After the sequence verifications, both $5^{\prime}-$ and $3^{\prime}$-homology arms were excised from the vectors by EcoRI digestions and then they were inserted via ligation inside the construct on the upstream of 'IRES-TauLacZ-pA' cassette and downstream of 'LoxP-pGK-NEO-pALoxP' cassette, respectively. These ligations were done in a blunt-ended manner with T4 Ligase (NEB) after blunting the 5'-sticky ends created by the restriction enzymes with Klenow (NEB) according to the manufacturers' protocols. The construct that contained all the necessary cassettes with the order of 'IRES-TauLacZ-pA', 'LoxPpGK-NEO-pA-LoxP', and 'pTK-pA', were kindly provided by Chowdhury K.

Prepared knockout construct was checked with restriction mapping by using several different restriction enzymes. The junction points where the ligations occurred were extensively checked with sequencings. The construct was linearized with AscI (NEB), purified with 'Phenol-Chloroform' method, precipitated with Ethanol and finally dissolved in TE. Integrity and concentration of the prepared DNA was checked 
by Agarose gel electrophoresis and spectrophotometer measurements. Then it was used for the electroporation of the construct into the ES-cells.

\section{\lrcorner$\underline{\text { Screening of mouse genomic } \lambda \text {-phage library }}$}

A $\lambda$-phage library (Lambda FixII by STRATAGENE) for the genomic DNA of mouse (129/SvJ strain) was screened with a 'PCR-based $\lambda$ DNA library screening' method as described in detail on the internet site:

(http://www.methods.info/Methods/Library screening/library pcr.pd f)

3 different overlapping $\lambda$-phage clones were isolated in different times with the corresponding primers:

PCR-primers for the $1^{\text {st }}$ screen:

\section{Au-181 : 5’- CTGGTCCTCGTCCTCATCTACT -3' \\ Au-183 : 5'- TGCAGCACCTATCAACATGTAA -3'}

PCR Conditions: Annealing temp is $58^{\circ} \mathrm{C}$, extension for 50 seconds.

PCR-primers for the $2^{\text {nd }}$ screen:

\section{Au-184 : 5’- CCTCTCACATGCTGCCCTTCTC -3' \\ Au-185 : 5’-ACGCCTTGATGgTCCTGGAAGT -3’}

PCR Conditions: Annealing temp is $64^{\circ} \mathrm{C}$, extension for 50 seconds.

PCR-primers for the $3^{\text {rd }}$ screen:

\section{Au-186 : 5'- TGGTACCAACCATACATCCACTGC -3' \\ Au-187 : 5’ GGAAGGCCATTTGCTTCTGGAG -3’}

PCR Conditions: Annealing temp is $66^{\circ} \mathrm{C}$, extension for 50 seconds.

Cycle numbers for PCR amplifications were changed according to the steps in $\lambda$ DNA library screening method.

The PCR fragments for all these 3 primer-pairs were also cloned into pGEMTeasy vector and sequence verified. Excised inserts for each were used as templates to prepare radioactively labeled probes with ${ }^{32} \mathrm{P}-\mathrm{dCTP}$ as described before.

After obtaining single positive $\lambda$-phage plaques, corresponding $\lambda$-DNA fragments were isolated by using the protocols described in Sambrook J. and Russell DW., (2001).

Obtained $\lambda$-DNA fragments were verified by restriction mapping and sequencing the $5^{\prime}$ - and $3^{\prime}$-ends. Afterwards, they were excised from the $\lambda$-phage arms with SalI and subcloned into the SalI sites of the pBluescript vector (Stratagene). 


\section{$\lesseqgtr \underline{\text { Second knockout construct }}$}

The second knockout construct (Figure 6B) was prepared for generating the conditional loss-of-function mutants for unc $5 \mathrm{~A}$.

$5^{\prime}$ - and internal- homology arms were excised from the sub-cloned $2^{\text {nd }} \lambda$-clone with HpaI - ClaI and ClaI - BclI digestions, respectively. 3'-homology arm was generated by excising the BclI-BclI partially digested fragment of sub-cloned $3^{\text {rd }} \lambda$ clone. 'pTK-pA' and 'FRT-pGK-NEO-pA-FRT-LoxP' cassettes were obtained from the departmental plasmid stocks. All the 5'- and 3'- sticky ends created by the restriction enzymes were filled-in by Klenow or T4 Polymerase (NEB) enzymes, respectively, according to the suppliers' protocols. Blunt-end ligations were used during these clonings

After extensive controls of the prepared knockout construct by restriction mapping and sequencing, it was linearized with PvuI enzyme (Promega). The linear construct, which was purified by 'Phenol-Chloroform' method and precipitated with ethanol, was finally dissolved in TE. Integrity and concentration of the prepared DNA was checked by Agarose gel electrophoresis and spectrophotometer measurements. Afterwards, it was used for the electroporation into the ES-cells.

\section{† $\underline{\text { Third knockout construct }}$}

The third knockout construct (Figure 6C) was same as the second construct except having only one 'TK' cassette instead of two. So, its preparation was almost in the same way as mentioned above for the second construct.

\section{$\rightarrow$ Fourth knockout construct}

The fourth knockout construct (Figure 6D) was prepared for generating the conventional loss-of-function mutants for unc5A. 5'-homology arm was generated by excising the NotI-FspI fragment from the sub-cloned $1^{\text {st }} \lambda$-clone, whereas a partially digested NotI-NheI fragment of sub-cloned $3^{\text {rd }} \lambda$-clone was used to generate the $3^{\prime}-$ homology arm. 'IRES-TauLacZ-pA' cassette was taken from a previously used construct (Mombaerts P. et.al., 1996). 'pTK-pA' and 'FRT-pGK-NEO-pA-FRT' cassettes were obtained from the departmental plasmid stocks. Similar to the previous constructs' preparations, all 5'- and $3^{\prime}$ - sticky ends created by the restriction enzymes 
were filled-in and blunt-end ligations were performed. The final construct was also extensively checked by sequencings and restriction mappings.

Linearization of this construct was done with AscI enzyme (NEB), and the purified linear construct was used for the two consecutive electroporations into the ES cells.

\section{Southern-blot screening of ES cell clones for homologous}

\section{$\underline{\text { recombination }}$}

After the electroporations of the knockout constructs into the ES-cells (MPI-II), they underwent a positive and negative selection for about 10 days with geneticin (G418) and gancyclovir, respectively. Individual ES-cells that survive during these selections were separately grown. While preparing the cryo-stocks of each clone, some from each were let to grow in 24 -well plates without the feeder layers. The screenings for the homologous recombination were done by using these ES cell cultures.

\section{$\neg$ Genomic DNA extraction from ES-cells}

Genomic DNAs from these ES cells were extracted by proteolytic digestion with Proteinase $\mathrm{K}(1 \mathrm{mg} / \mathrm{ml})$ in Lysis Buffer at $56^{\circ} \mathrm{C}$ for overnight and then precipitating the genomic DNA with the addition of $1.5 x$ volume Isopropanol onto the lysed samples. After vigorous shaking by hand, they were centrifuged for 20 minutes, and then the pellets were washed with $70 \%$ ethanol and finally dissolved in $100 \mu \mathrm{l}$ of TE.

Lysis Buffer (final concentrations of the ingredients):

$\begin{array}{ll}100 \mathrm{mM} & \text { Tris-Cl (pH 8.0) } \\ 5 \mathrm{mM} & \text { EDTA (pH 8.0) } \\ 0.2 \% & \text { SDS } \\ 200 \mathrm{mM} & \mathrm{NaCl}\end{array}$




\title{
$\Rightarrow$ Digestions of genomic DNA and southern-blotting
}

During the preparation of all four different knockout constructs mentioned above, some EcoRI sites were introduced in specific regions of the construct. These EcoRI sites are absent in the wildtype genomic region. In case of homologous recombination, digestion with EcoRI would therefore produce specific bands of expected sizes as shown in Figure 6D and 6E.

With this idea, one third of each ES-clone genomic DNA sample was digested with EcoRI (Promega) at $37^{\circ} \mathrm{C}$ overnight. Digested genomic DNA samples were loaded onto $0.7 \%$ Agarose gels and electrophoresed at 30V overnight. Southern-blotting of these gels were done according to the protocols in Sambrook J. and Russell DW, (2001) in order to transfer the DNA fragments on the gels to the nitrocellulose membranes.

\section{$\rightarrow$ Probes for southern-blot hybridizations}

Particular genomic fragments (shown as small red rectangles in Figures 6A-D) were PCR amplified from the wildtype ES-cell genomic DNA with the following primer-pairs:

\section{Au-518_Frw : 5'-TACACAAgAgCATCTTGCAgGT -3' Au-518_Rev : 5'-CTTCCTTCTCTCTGGTGTGTGA -3’ \\ PCR Conditions: Annealing temp is $60^{\circ} \mathrm{C}$, extension for 50 seconds.}

\author{
Au-318_Frw : 5'-AgAgggatagCAAgAgggagag -3' \\ Au-318_Rev : 5'- GCTTTTATCCGTTTTGACCTTG -3' \\ PCR Conditions: Annealing temp is $60^{\circ} \mathrm{C}$, extension for 50 seconds.
}

\section{Au-5int_Frw : 5' - TTCACTGgGTTTTTCTCGATGC -3' Au-5int_Rev : 5'-GgTCTCCCTGACAACCCAGAAT-3' \\ PCR Conditions: Annealing temp is $60^{\circ} \mathrm{C}$, extension for 50 seconds.}

The corresponding 1.0, 1.0, 1.5 kb PCR fragments, respectively, were purified, and cloned into pGem-Teasy vector. After sequence verifications, the inserts were excised with NotI digestion, and purified DNA fragments were used as templates for radioactively labeled Southern-blot-hybridization probes with the protocols mentioned 
before. 'Au-318' was used as 3'-external probe in all screens with all four constructs. 'Au-518' served as a 5'-external probe in the screens for the first three constructs, whereas 'Au-5int' was used as 5'-internal probe in the screens for the last construct.

\section{isouthern-blot hybridizations}

All the southern-blot hybridizations were done according to the protocols in Sambrook J. and Russell DW., (2001) with the corresponding labeled probes.

All ES-cell clones were always checked by both 5'- and $3^{\prime}$ - probes.

The positive ES-cell clones were further checked intensively by using different restriction enzymes for the digestion of their genomic DNA, and additional internal and external hybridization probes.

\section{Generation of chimaeras and screening for germline}

\section{$\underline{\text { transmissions }}$}

The chimaeras were produced from the positive ES-cell clones either by 'aggregation' with the isolated morulas or by 'blastocyst injection', in which the EScells were injected into the isolated blastocysts.

Production of chimaeras by 'aggregation' was done in the departmental facility by U.Franke. The 'blastocyst injection' methods were kindly performed by the transgenic facility of 'Max-Planck Institute for Experimental Medicine', Goettingen.

The chimaerism for the chimaeras were roughly determined according to the percentage of agouti color within the total fur color.

For germline-transmission screens, the chimaeras were mated with CD1-outbreed strain partners. Eye and/or fur color of the progeny from these matings were used as phenotypic signs of germline transmission. These matings continued till reaching the approximately 80 negative pups for each chimera before concluding that this chimera is negative in terms of germline transmission.

ES-cells were originally isolated from (129/Sv) inbred strain of mouse embryos. This strain has 'black eye' and 'agouti coat color' features. The morulas used for the aggregation were isolated from CD1 or NMRI outbreed strains, which have 'red eyes' and 'white coat colors'. Black eye and agouti coat color are dominant traits over the red 
eye and white coat color features. Therefore, the chimaeras that have germ cells originated from the aggregated ES-cells should give progeny with black eye and agouti coat color. None of the aggregation-produced chimaeras gave such pups.

The blastocysts used for the blastocyst injection were isolated from C57/BL6 strain of mice. Since black coat color is recessive over agouti coat color, the germline transmission would require the production of pups with agouti coat colors. Eye colors can not be used as phenotypic evidence of germline transmission, because all the mice generated by this blastocyst injection will transmit the 'black eye' feature to their progeny independent of their chimaerism or germline transmission success. Some of the chimaeras produced by blastocyst injection of ES\#110 clone gave progeny with agouti coat colors. As expected, half of these agouti pups were genotyped as heterozygotes for unc $5 \mathrm{~A}$ gene by both Southern Blot and PCR analysis.

\section{$\rightarrow \underline{P C R \text {-genotyping method for unc5A knockout mice }}$}

All mouse or embryos were genotyped by using the isolated genomic DNA from their tails with the PCR primers:

GN-A : 5'-ATCAACCATTTGgGACAgGgAGAC -3’

GN-B : 5'- ATGTTCTTGgCCACGCAGgTGTAG -3'

GN-C : 5'- GGGGGCGGAATTCTTAATTAACTCG -3'

PCR conditions: Annealing temp is $67^{\circ} \mathrm{C}$, extension for 50 seconds.

Wildtype allele generates an 875 bps band, whereas the mutant allele generates a 459 bps band (Figure 7A).

\section{\} \underline { \text { Genomic DNA extraction from cut-tails } }}

Genomic DNA samples from the cut-tails were extracted by proteolytic digestion of tails with Proteinase $\mathrm{K}(1 \mathrm{mg} / \mathrm{ml})$ in Lysis Buffer at $56^{\circ} \mathrm{C}$ for 3 hours and then precipitating the genomic DNA with the addition of $1.5 \mathrm{x}$ volume Isopropanol onto the lysed samples. After vigorous shaking by hand, they were centrifuged for 20 minutes, and then the pellets were washed with $70 \%$ ethanol and finally dissolved in $100 \mu 1$ of TE. $1 \mu l$ of each DNA sample was used in PCR genotypings. 


\section{Investigation of general health and behavioral aspects of unc5A}

\section{knockout mice}

The distributions of the three genotypes among the pups from the F1 matings were counted. It was observed for both $129 x \mathrm{xD} 1$ mixed and 129 pure backgrounds that the distributions were compatible with the Mendelian transmission ratio $(25 \%$ homozygote, $50 \%$ heterozygote, and $25 \%$ wildtype).

10 homozygote mice ( 5 male and 5 female) were mated with CD1 mating partners in order to observe their fertility. No reproductive problems were observed.

Meanwhile, 5 female wildtype littermates of the homozygote females above were also mated with CD1 males and nursing behaviors of the homozygous mothers were followed in comparison with the wildtype littermates. The growth and survival rates of the pups, the behavior of making nests, and the attitude of collecting the pups to the nest after they are being spread within the cage were observed till their pups reached the age of PN-15.

All the basic behavioral observations for visual, auditory and olfactory sensory systems were done according to the methods described in Crawley JN, 2000.

\section{Histological analysis of brains}

Dissected postnatal mouse brains were fixed overnight in 4\% PFA and washed in $0.86 \% \mathrm{NaCl}$ for a day. Dehydration of brains was done through increasing ethanol solutions $(50 \%, 70 \%, 80 \%, 90 \%, 96 \%$, and $100 \%)$ by changing daily. After a further day of incubation in isopropanol, they were transferred into Toluol for 6 hours of incubation. All the incubations before toluol were done at $4^{\circ} \mathrm{C}$, and Toluol at room temperature. Afterwards, brains were transferred into melted-Paraplast (Tycon Healthcare) at $56^{\circ} \mathrm{C}$ for two days and then embedded into paraffin blocks for coronal sectioning with Microtome (Leica Instruments). $12 \mu \mathrm{m}$ thick sections were prepared and air-dried for couple of hours.

Cresyl-Violet staining of paraffin sections was done according to the protocols described in Paxinos G. and Watson C., 1986; and Davenport HA., 1960. 


\section{RT-PCR analysis for confirming the success of unc $5 A$ targeting}

\section{construct}

RNA isolation from PN-1 pups' head samples and cDNA synthesis from these RNA was done as described before.

PCR analyses on these cDNA samples were done with gene-specific primers for unc $5 A$ (Au-18cF and $\mathrm{Au}-18 \mathrm{cR}$ ) and $L a c Z$ with expected sizes of 423 bps and $899 \mathrm{bps}$, respectively.

\section{Au-18cF : 5'- GGAGATGGACAGAGCTTCAACA -3' \\ Au-18cR : 5’-CTgGTGAgAgtgtaggCGTGACC -3’ \\ PCR conditions: Annealing temp is $64^{\circ} \mathrm{C}$, extension for 50 seconds. \\ LacF : 5'-ATATTATTTGCCCGATGTACGC $-3^{\prime}$ \\ LacR : 5’-ACTTACGCCAATGTCGTTATCC -3’ \\ PCR conditions: Annealing temp is $60^{\circ} \mathrm{C}$, extension for 50 seconds.}

\section{Generation of conditional-overexpression construct}

The IMAGE clone (Genbank Acc: CA318180), which contains the full-length cDNA sequence of " $\Delta$ exon6" spliced variant for unc $5 A$ gene, was obtained from RZPD,Germany. It was sequenced extensively through the whole insert sequence to confirm the open-reading frame and to ensure that it does not have any mutations. 'IRES-TauLacz-pA' cassette was kindly provided by Chowdhury K. This cassette was cloned on to the 3'-side of unc5A-Aexon6 coding sequences via blunt-end ligation. Afterwards, the whole insert containing the cDNA sequence for unc5A-Aexon6 and IRES-TauLacz-pA' cassette was excised out and cloned on to the 3'-end of the conditional-overexpression construct's insert. This conditional-overexpression construct (Sawicki JA. et.al., 1998) had all the cassettes with 5'-to-3'- order: a composit CMV-IE and $\mathrm{B}$-actin promoter, $\mathrm{B}$-actin intron, and floxed eGFP sequences that contains several 'PolyA' signal sequences at the end. 
After verification of the correct cloning of the construct by restriction mappings and sequencings, it was prepared for microinjection by SalI-excision of the whole insert.

\section{Generation of mouse lines for conditional-overexpression of}

\section{$\underline{\text { UNC5A- } \Delta \operatorname{exon6}}$}

Microinjection of the generated construct into the pronuclei of fertilized eggs (B6CBA inbred strain) was done in the departmental facility by a highly experienced person. The founder lines from these microinjections were screened initially by PCR on isolated genomic DNA from the cut-tails. 3 different primer pairs were used for the screen. They were designed from the CMV promoter, unc $5 A-\Delta e x o n 6 \mathrm{cDNA}(\mathrm{Au}-18 \mathrm{cF}$ and $\mathrm{Au}-18 \mathrm{cR}$, mentioned before) and $\mathrm{LacZ}$ gene (mentioned before) regions:

\section{CMV-F : 5’- TACGGGGTCATTAGTTCATAGC $-\mathbf{3}^{\prime}$ \\ CMV-R : 5'- GCCAAGTAGGAAAGTCCCATAA -3'}

PCR conditions: Annealing temp is $60^{\circ} \mathrm{C}$, extension for 50 seconds.

Positive founders were also genotyped with Southern-blot analysis using different types of restriction enzymes and hybridization probes in order to ensure that all parts of the construct were integrated correctly.

The positive founder mice were mated with C57/BL6-N mating partners, and progeny from these matings were analyzed for uniform eGFP expression in several dissected organs under a fluorescent microscope (Olympus SZX12).

The genotyping of the established lines during the backcrossings and experimental analyses were all done by checking the eGFP expression under an UVfluorescence microscope. 


\section{PCR genotypings of CRE- and reporter mouse lines}

All genotypings for colony maintenance and experimental analyses for the CRE and reporter lines were done by using the isolated genomic DNA from the cut-tail samples.

ECG-Cre and Nex-Cre mouse lines were genotyped by PCR with CRE-specific primers:

\section{CRE-F : 5’-ATGCTTCTGTCCGTTTGCCG -3’ \\ CRE-R : 5'- CCTGTTTTGCACGTTCACCG -3’}

PCR conditions: Annealing temp is $57^{\circ} \mathrm{C}$, extension for 30 seconds.

The reporter Floxed-ROSA mouse line was genotyped by PCR with LacZspecific primers that were mentioned before.

\section{Whole-mount LacZ stainings}

Embryos were harvested as required and fixed in FixB solution for 50 minutes (E12.5 embryos) or 60 minutes (E13.5 embryos). They were washed twice with $1 \mathrm{X}$ PBS for 20 minutes each, and then stained in $\mathrm{X}-\mathrm{Gal}$ staining solution at $30^{\circ} \mathrm{C}$ as long as it is necessary (usually 20 to 40 hours). Stainings were stopped with washing twice in $1 \mathrm{X}$ PBS for 30 minutes each.

FixB solution (final concentrations of the ingredients):

$\begin{array}{ll}1 \% & \text { Formaldehyde } \\ 0.2 \% & \text { Glutaraldehyde } \\ 0.2 \% & \text { NP-40 } \\ 0.1 \% & \text { SDS } \\ 1 \mathrm{X} & \text { PBS }\end{array}$

$X$-Gal staining solution (final concentrations of the ingredients):

$\begin{array}{ll}1 \mathrm{mg} / \mathrm{ml} & \mathrm{X}-\mathrm{GAL} \\ 5 \mathrm{mM} & \mathrm{K}_{3} \mathrm{Fe}(\mathrm{CN})_{6} \\ 5 \mathrm{mM} & \mathrm{K}_{4} \mathrm{Fe}(\mathrm{CN})_{6} \\ 2 \mathrm{mM} & \mathrm{MgCl}_{2} \\ 1 \mathrm{X} & \mathrm{PBS}\end{array}$




\section{LacZ stainings on cryosections}

E12.5 embryos were harvested and immediately embedded in 'Tissue Freezing Medium' (from 'Jung') on dry ice. The sections were prepared with a cryostat (Leica), and taken onto 'SuperFrost Plus' slides (Menzel-Glaser). After 1 hours of air-drying they were fixed in $0.2 \%$ Glutaraldehyde and then washed three-times with LacZ Buffer for 5 minutes each. Afterwards they are put in X-Gal staining solution, which was described above. Stainings were done at $30^{\circ} \mathrm{C}$ as long as it is necessary (usually 48 to 96 hours) and were stopped by washing twice within 1X PBS for 30 minutes each.

LacZ Buffer (final concentrations of the ingredients):

$\begin{array}{ll}2 \mathrm{mM} & \mathrm{MgCl}_{2} \\ 0.02 \% & \mathrm{NP}-40 \\ 0.01 \% & \mathrm{NaDOC} \\ 1 \mathrm{X} & \mathrm{PBS}\end{array}$

\section{Transfection of Neuro2A cells and LacZ staining analyses}

Neuro2A cells were cultured in DMEM with $10 \%$ fetal bovine serum. These cells were transiently transfected with the indicated plasmids using Lipofectamine (Invitrogen) according to the manufacturer's instructions.

48 hours after transfection, eGFP expression was checked under the fluorescence microscope and then LacZ staining analysis was done as following:

The cells were washed twice with 1XPBS and fixed with SolB for 5 minutes at room temperature. Then, they were washed three times with SolC for 5 minutes each, stained in SolD at $37^{\circ} \mathrm{C}$ as long as it is necessary and were stopped by washing twice within 1X PBS for 10 minutes each.

SolB (final concentrations of the ingredients):

$\begin{array}{ll}0.2 \% & \text { Glutaraldehyde } \\ 5 \mathrm{mM} & \text { EGTA } \\ 2 \mathrm{mM} & \mathrm{MgCl}_{2} \\ 100 \mathrm{mM} & \mathrm{K}_{2} \mathrm{HPO}_{4}\end{array}$


SolC (final concentrations of the ingredients):

$\begin{array}{ll}5 \mathrm{mM} & \text { EGTA } \\ 2 \mathrm{mM} & \mathrm{MgCl}_{2} \\ 0.02 \% & \mathrm{NP}-40 \\ 0.01 \% & \mathrm{NaDOC} \\ 100 \mathrm{mM} & \mathrm{K}_{2} \mathrm{HPO}_{4}\end{array}$

SolD (final concentrations of the ingredients):

$\begin{array}{cl}0.5 \mathrm{mg} / \mathrm{ml} & \mathrm{X}-\mathrm{GAL} \\ 10 \mathrm{mM} & \mathrm{K}_{3} \mathrm{Fe}(\mathrm{CN})_{6} \\ 10 \mathrm{mM} & \mathrm{K}_{4} \mathrm{Fe}(\mathrm{CN})_{6} \\ 5 \mathrm{mM} & \mathrm{EGTA} \\ 2 \mathrm{mM} & \mathrm{MgCl}_{2} \\ 0.02 \% & \mathrm{NP}-40 \\ 0.01 \% & \mathrm{NaDOC} \% \\ 100 \mathrm{mM} & \mathrm{K}_{2} \mathrm{HPO}_{4}\end{array}$




\section{ABBREVIATIONS}

All the abbreviations for the names of genes/proteins and chemical entities were used in accordance with the standard nomenclature.

$\begin{array}{ll}\text { Acc. } & \text { Accession } \\ \text { bps } & \text { base pairs } \\ \text { CMV } & \text { Cyto-Megalo Virus } \\ \text { CNS } & \text { Central Nervous System } \\ \text { cDNA } & \text { complementary DNA } \\ \text { DEPC } & \text { diethyl-PyroCarbonate } \\ \text { dig } & \text { digoxygenin } \\ \text { ECM } & \text { Extracellular matrix } \\ \text { eGFP } & \text { enhanced Green Fluorescence Protein } \\ \text { En } & n \text { indicates the embryonic day } \\ \text { ES-cell } & \text { Embryonic stem cell } \\ \text { EST } & \text { Expressed sequence tag } \\ \text { IRES } & \text { Internal ribosome entry site } \\ \text { ISH } & \text { In situ hybridization } \\ \text { kb } & \text { kilo basepairs } \\ \text { kDA } & \text { kiloDalton } \\ \text { LacZ } & \text { Peta-galactosidase } \\ \text { LOA } & \text { Lateral Olfactory Area } \\ \text { LOH } & \text { Loss Of Heterozygoty } \\ \text { mRNA } & \text { messenger RNA } \\ \text { MOA } & \text { Marginal Zone } \\ \text { MZ } & \text { Open-Reading-Frame } \\ \text { N } n & \text { PCR }\end{array}$




$\begin{array}{ll}\text { PN } & \text { Post-Natal } \\ \text { RACE } & \text { Rapid Amplification of CDNA Ends } \\ \text { RMS } & \text { Rostral Migratory Stream } \\ \text { RT } & \text { Reverse Transcription } \\ \text { SSC } & \text { Standard Saline Citrate solution } \\ \text { SVZ } & \text { SubVentricular Zone } \\ \text { TBE } & \text { Tris-Boric acid- EDTA buffer } \\ \text { TCA } & \text { ThalamoCortical Axon } \\ \text { TE } & \text { Tris-EDTA buffer } \\ \text { TK } & \text { Thymidine Kinase } \\ \text { TSP } & \text { ThromboSPondin } \\ \text { UTR } & \text { UnTranslated Region } \\ \text { UV } & \text { UltraViolet } \\ \text { VZ } & \text { Ventricular Zone } \\ \text { WT } & \text { WildType } \\ \text { X-Gal } & \text { 5-Bromo-4-chloro-3-indolyl- } \beta \text {-D-galactoside }\end{array}$




\section{REFERENCES}

Ackerman S.L., Kozak L.P., Przyborski S.A., Rund L.A., Boyer B.B., Knowles B.B. (1997) The mouse rostral cerebellar malformation gene encodes a UNC-5-like protein. Nature (386): 838-842

Augsburger A., Schuchardt A., Hoskins S., Dodd J., Butler S. (1999) BMPs as mediators of roof plate repulsion of commissural neurons. Neuron (24): 127-141

Auld V. (1999) Glia as mediators of growth-cone guidance: studies from insect nervous system. Cell Mol Life Sci. (55): 1377-1385

Bagri A., Marin O., Plump A.S., Mak J., Pleasure S.J., Rubenstein J.L., TessierLavigne M. (2002) Slit proteins prevent midline crossing and determine the dorsoventral position of major axonal pathways in the mammalian forebrain. Neuron (33):233-248

Barallobre M.J., Del Rio J.A., Alcantara S., Borrell V., Aguado F., Ruiz M., Carmona M.A., Martin M., Fabre M., Yuste R., Tessier-Lavigne M., Soriano E. (2000) Aberrant development of hippocampal circuits and altered neural activity in netrin-1-deficient mice. Development (127) :4797-4810

Barallobre M.J., Pascual M., del Rio J.A., Soriano E. (2005) The netrin family of guidance factors: emphasis on Netrin-1 signaling. Brain Res Brain Res Rev (49):2247

Bentley D. and O'Connor T.P. (1994) Cytoskeletal events in growth cone steering. Curr Opin Neurobiol. (4): 43-48 
Bigbee JW., Sharma KV., Gupta JJ., Dupree JL. (1999) Morphogenic role for acetylcholinesterase in axonal outgrowth during neural development. Environ Health Perspect (107 Suppl1): 81-87

Braisted J.E., Catalano S.M., Stimac R., Kennedy T.E., Tessier-Lavigne M., Shatz C.J., O'Leary D.D. (2000) Netrin-1 promotes thalamic axon growth and is required for proper development of the thalamocortical projection. J Neurosci. (20): 57925801

Brose K. and Tessier-Lavigne M. (2000) Slit proteins: key regulators of axon guidance, axonal branching, and cell migration. Curr Opin Neurobiol. (10): 95-102

Bruckner K., Pasquale E.B., Klein R. (1997) Tyrosine phosphorylation of transmembrane ligands for Eph receptors. Science (275): 1640-1643

Burgess RW., Jucius TJ., Ackerman SL. (2006) Motor axon guidance of the mammalian trochlear and phrenic nerves: dependence on the netrin receptor Unc5c and modifier loci. J Neurosci. (26): 5756-5766.

Burki F. and Kaessmann H. (2004) Birth and adaptive evolution of a hominoid gene that supports high neurotransmitter flux. Nat Genet (36): 1061-1063

Chan S.S., Zheng H., Su M.W., Wilk R., Killeen M.T., Hedgecock E.M., Culotti J.G. (1996) UNC-40, a C.elegans homolog of DCC, is required in motile cells responding to UNC-6 netrin cues. Cell (87):187-195

Chilton J.K. (2006) Molecular mechanisms of axon guidance. Dev Biol. (292): $13-24$

Crawley JN. (2000) What's wrong with my mouse? John Wiley and Sons, Inc. publications. 
Davenport HA. (1960) Histological and histochemical techniques. W.B. Saunders

De Carlos J.A. and O'Leary D.D. (1992) Growth and targeting of subplate axons and establishment of major cortical pathways. J Neurosci. (12): 1194-1211

Deiner M.S., Kennedy T.E., Fazeli A., Serafini T., Tessier-Lavigne M., Sretawan D.W. (1997) Netrin-1 and DCC mediate axon guidance locally at the optic disc: loss of function leads to optic nerve hypoplasia. Neuron (19): 575-589

Dent E.W. and Gertler F.B. (2003) Cytoskeletal dynamics and transport in growth cone motility and axon guidance. Neuron (40): 209-227

Duysen EG., Lockridge O. (2006) Phenotype comparison of three acetylcholinesterase knockout strains. J Mol NeuroscI. (30): 91-92

Engelkamp D. (2002) Cloning of three mouse Unc5 genes and their expression patterns at mid-gestation. Mech Dev. (118): 191-197

Fazeli A., Dickinson S.L., Hermiston M.L., Tighe R.V., Steen R.G., Smal C.G., Stoeckli E.T., Keino-Masu K., Masu M., Rayburn H., Simons J., Bronson R.T., Gordon J.I., Tessier-Lavigne M., Weinberg R.A. (1997) Phenotype of mice lacking functional deleted in colorectal cancer (dcc) gene. Nature (386): 796-804

Fearon E.R., Cho K.R., Nigro J.M., Kern S.E., Simons J.W., Ruppert J.M., Hamilton S.R., Preisinger A.C., Thomas G., Kinzler K.W. (1990) Identification of a chromosome 18q gene that is altered in colorectal cancers. Science (247): 49-56

Feldheim D.A., Kim Y.I., Bergemann A.D., Frisen J., Barbacid M., Flanagan J.G. (2000)

Genetic analyses of ephrin-A2 and ephrin-A5 shows their requirement in multiple aspects of retinocollicular mapping. Neuron (25): 563-574 
Feng Y. and Walsh CA.(2004) Mitotic spindle regulation by Nde1 controls cerebral cortical size. Neuron. (44):279-293.

Finger J.H., Bronson R.T., Harris B., Johnson K., Przyborski S.A., Ackerman S.L. (2002) The netrin-1 receptors UNC5h3 and DCC are necessary at multiple choice points for the guidance of corticospinal tract axons. J Neurosci. (22): 10346-10356

Fournier A.E., Nakamura F., Kawamoto S., Goshima Y., Kalb R.G., Strittmatter S.M. (2000) Semaphorin3A enhances endocytosis at sites of receptor-F-actin colocalization during growth cone collapse. J Cell Biol. (149): 411-422

Fujisawa H. and Kitsukawa T. (1998) Receptors for collapsin/semaphorins. Curr Opin Neurobiol. (8): 587-592

Gad J.M., Keeling S.L., Wilks A.F., Tan S.S., Cooper H.M. (1997) The expression patterns of guidance receptors, DCC and Neogenin, are spatially and temporally distinct throughout mouse embryogenesis. Dev Biol. (192): 258-273

Geisbrecht B.V., Dowd K.A., Barfield R.W., Longo P.A., Leahy D.J. (2003) Netrin binds discrete subdomains of DCC and UNC5 and mediates interactions between DCC and heparin. J Biol Chem. (278): 32561-32568

Ghosh A and Shatz C.J. (1992) Pathfinding and target selection by developing geniculocortical axons. $J$ Neurosci. (12): 39-55

Ghosh A.and Shatz C.J. (1993) A role for subplate neurons in the patterning of connections from thalamus to neocortex. Development (117): 1031-1047

Goebbels S., Bormuth I., Bode U., Hermanson O., Schwab MH., Nave KA. (2006) Genetic targeting of principal neurons in neocortex and hippocampus of Nex-Cre mice. Genesis (44): 611-621 
Goldberg D.J. and Burmeister D.W. (1986) Stages in axon formation: observations of growth of Aplysia axons in culture using video-enhanced contrastdifferential interference contrast microscopy. J Cell Biol. (103): 1921-1931

Goldberg D.J. and Burmeister D.W. (1989) Looking into growth cones. Trends Neurosci. (12): 503-506

Guyton A.C., Hall J.E. (1996) Textbook of Medical Physiology. W.B.Saunders Company, Pennsylvania.

Hall A.C., Lucas F.R., Salinas P.C. (2000) Axonal remodeling and synaptic differentiation in the cerebellum is regulated by Wnt-7a signaling. Cell (100): 525 535

Hedgecock E.M., Culotti J.G., Hall D.H. (1990) The unc-5, unc-6, and unc-40 genes guide circumferential migrations of pioneer axons and mesodermal cells on thE epidermis in C.elegans. Neuron (4): 61-85

Hevner R.F., Miyashita-Lin E., Rubenstein J.L.R. (2002) Cortical and thalamic axon pathfinding defects in Tbr1, gbx2, and Pax6 mutant mice: Evidence that cortical and thalamic axons interact and guide each other. J Comp NeuroL. (447): $8-17$

Holland S.J., Gale N.W., Mbamalu G., Yancopoulos G.D., Henkemeyer M., Pawson T. (1996) Bidirectional signaling through the EPH-family receptor Nuk and its transmembrane ligands. Nature (383): 722-725

Hu Z., Yue X., Shi G., Yue Y., Crockett D.P., Blair-Flynn J., Reuhl K., Tessarollo L., Zhou R. (2003) Corpus callosum deficiency in transgenic mice expressing a truncated ephrin-A receptor. J Neurosci. (23): 10963-10970

Huot J. (2004) Ephrin signaling in axon guidance. Prog Neuropsychopharmacol Biol Psychiatry (28): 813-818 
Innocenti G.M. and Price D.J.(2005) Exuberrance in the development of cortical networks. Nat Rev Neurosci. (6): 955-965

JAX mice Data Sheet for B6CBA. (http://jaxmice.jax.org/strain/0009556 3.html) The Jackson Laboratory.

JAX mice Data Sheet for FVB/NJ. (http://jaxmice.jax.org/strain/001800.html) The Jackson Laboratory.

JAX Notes. Genetic Background effects: Can your mice see? (Spring 2002) The Jackson Laboratory. (http://jaxmice.jax.org/library/notes/485.pdf) pp:2-3

Job C. and Tan SS. (2003) Constructing the mammalian neocortex: the role of intrinsic factors. Dev Biol. (257): 221-232

Kaas JH. (2004) Evolution of somatosensory and motor cortex in primates. Anat Rec A Discov Mol Cell Evol Biol. (281): 1148-1156

Kennedy T.E., Serafini T., de la Torre J.R., Tessier-Lavigne M. (1994) Netrins are diffusible chemotrophic factors for commissural axons in the embryonic spinal cord. Cell (78): 425-435

Kidd T., Brose K., Mitchell K.J., Fetter R.D., Tessier-Lavigne M., Goodman C.S., Tear G. (1998) Roundabout controls axon crossing of the CNS midline and defines a novel subfamily of evolutionary conserved guidance receptors. Cell (92): 205-215

Kolodkin A.L., Matthes D.J., Goodman C.S. (1993) The semaphorin genes encode a family of transmembrane and secreted growth cone guidance molecules. Cell (75):1389-1399

Kullander K. and Klein R. (2002) Mechanisms and functions of Eph and ephrin signaling. Nat Rev Mol Cell Biol. (3): 475-486 
Leonardo E.D., Hinck L., Masu M., Keino-Masu K., Ackerman S.L., TessierLavigne M. (1997) Vertebrate homologues of C.elegans UNC-5 are candidate netrin receptors. Nature (386): 833-838

Llambi F., Causeret F., Bloch-Gallego E., Mehlen P. (2001) Netrin-1 acts as a survival factor via its receptors UNC5H and DCC. EMBO J (20): 2715-2722

Lu X., LeNoble F., Yuan L., Jiang Q., De Lafarge B., Sugiyama D., Breant C., Claes F., DeSmet F., Thomas JL., Autiero M., Carmeliet P., Tessier-Lavigne M., Eichmann A. (2004) The netrin receptor UNC5B mediates guidance events controlling morphogenesis of the vascular system. Nature (432):179-186

Mao X. Fujiwara Y., Orkin SH. (1999) Improved reporter strain for monitoring Cre recombinase-mediated DNA excisions in mice. Proc Natl Acad Sci U.S.A. (96): 5037-5042

Marquardt T., Ashery-Padan R., Andrejewski N., Scardigli R., Guillemot F., Gruss P. (2001) Pax6 is required for the multipotent state of retinal progenitor cells. Cell (105): 43-55

McConnell SK, Kaznowski CE. (1991) Cell cycle dependence of laminar determination in developing neocortex. Science. (254): 282-285.

McConnell SK. (1995) Strategies for the generation of neuronal diversity in the developing central nervous system. J Neurosci. (15): 6987-6998.

Mehlen P. and Furne C. (2005) Netrin-1: when a neuronal guidance cue turns out to be a regulator of tumorigenesis. Cell Mol Life Sci. (62): 2599-2616

Molnar Z. and Blakemore C. (1995) How do thalamic axons find their way to the cortex? Trends Neurosci. (18): 389-397 
Molnar Z., Metin C., Stoykova A., Tarabykin V., Price D.J.,Francis F., Meyer G.,Dehay C., Kennedy H. (2006) Comparative aspects of cerebral cortical development. Eur J Neurosci (23): 921-934

Mombaerts P., Wang F., Dulac C., Chao SK., Nemes A., Mendelsohn M., Edmondson J., Axel R. (1996) Visualizing an olfactory sensory map. Cell (87): 675686

Mueller B.K. (1999) Growth cone guidance: first steps towards a deeper understanding. Annu Rev Neurosci. (22): 351-388

Muzio L. and Mallamaci A. (2003) Emx1, emx2, and pax6 in specification, regionalization, and arealization of the cerebral cortex. Cereb Cortex. (13): 641-647

O’Leary D.D. (1989) Do cortical areas emerge from a protocortex? Trends Neurosci. (12): 400-406

O’Leary D.D. and Nakagawa Y. (2002) Patterning centers, regulatory genes, and extrinsic mechanisms controlling arealization of the neocortex. Curr Opin Neurobiol. (12): 14-25

Ozdinler P.H. and Erzurumlu R.S. (2002) Slit-2, a branching-arborization factor for sensory axons in the Mammalian CNS. J Neurosci. (22): 4540-4549

Paxinos G. (1985) The Rat nervous system: Forebrain and midbrain. (vol1) Academic Press, Australia.

Paxinos G. and Watson C. (1986) The rat brain in stereotaxic coordinates. $2^{\text {nd }}$ edition, Academic Press

Plachez C. and Richards L.J. (2005) Mechanisms of axon guidance in the developing nervous system. Curr Top Dev Biol (69): 267-346 
Ponting C. and Jackson AP. (2005) Evolution of primary microcephaly genes and the enlargement of primate brains. Curr Opin Genet Dev (15):241-248

Price D.J., Kennedy H., Dehay C., Zhou L., Mercier M., Jossin Y., Goffinet A.M., Tissir F., Blakey D., Molnar Z. (2006) The development of cortical connections. Eur J Neurosci. (23): 910-920

Rakic P. (1988) Specification of cerebral cortical areas. Science (241):170-176

Rakic P. (2003) Developmental and evolutionary adaptations of cortical radial glia. Cereb Cortex. (13): 541-549

Rash B.G. and Richards L.J. (2001) A role for cingulate pioneering axons in the development of the corpus callosum. J Comp Neurol. (434): 147-157

Rolls E.T. (2004) The functions of the orbitofrontal cortex. Brain Cogn. (55): $11-29$

Roth G. and Dicke U. (2005) Evolution of the brain and intelligence. Trends Cogn Sci. (9): 250-257

Rubenstein J.L., Anderson S., Shi L., Miyashita-Lin E., Bulfone A., Hevner R. (1999) Genetic control of cortical regionalization and connectivity. Cereb Cortex. (9): 524-532

Sabry J.H., O’Connor T.P., Evans L., Toroian-Raymond A., Kirschner M., Bentley D. (1991) Microtubule behavior during guidance of pioneer neuron growth cones in situ. J Cell Biol. (115): 381-395

Sambrook J. and Russell DW. (2001) Molecular Cloning. Third edition. Cold Spring Harbor Laboratory Press, NewYork. 
Sasaki S, Mori D, Toyo-oka K, Chen A, Garrett-Beal L, Muramatsu M, Miyagawa S, Hiraiwa N, Yoshiki A, Wynshaw-Boris A, Hirotsune S. (2005) Complete loss of Ndel1 results in neuronal migration defects and early embryonic lethality.

Mol Cell Biol. 25(17): 7812-7827.

Sawicki JA., Morris RJ., Monks B., Sakai K. and Miyazaki J. (1998) A composite CMV-IE enhancer/b-actin promoter is ubiquitously expressed in mouse cutaneous epithelium. Exp Cell Res. (244): 367-369

Schoenbaum G., Roesch M. (2005) Orbitofrontal cortex, associative learning, and experiences. Neuron (47): 633-636

Serafini T., Kennedy T.E., Galko M.J., Mirzayan C., Jessell T.M., TessierLavigne M. (1994) The netrins define a family of axon outgrowth-promoting proteins homologous to C.elegans UNC-6. Cell (78): 409-424

Serafini T., Colamarino S.A., Leonardo E.D., Wang H., Beddington R., Skarnes W.C., Tessier-Lavigne M. (1996) Netrin-1 is required for commissural axon guidance in the developing vertebrate nervous system. Cell (87): 1001-1014

Srinivasan K., Strickland P., Valdes A., Shin G.C., Hinck L. (2003) Netrin1/Neogenin interaction stabilizes multipotent progenitor cap cells during mammary gland morphogenesis. Dev Cell. (4): 371-382

Tanikawa C., Matsuda K., Fukuda S., Nakamura Y., Arakawa H. (2003) p53RDL1 regulates p53-dependent apoptosis. Nat Cell Biol. (5): 216-223

Tessier-Lavigne M. and Goodman C.S. (1996) The molecular biology of axon guidance. Science (274): 1123-1133

Toran-Allerand C.D. (2004) Estrogen and the brain: Beyond ER-alpha and ER-beta. Exp Gerontol (39): 1579-1586 
Wahlsten D., Metten P., Crabbe JC. (2003) Survey of 21 inbred mouse strains in two laboratories reveals that BTBR $\mathrm{T} 7+\mathrm{tf} / \mathrm{tf}$ has severely reduced hippocampal commissure and absent corpus callosum. Brain Res. (971): 47-54

Wahlsten D., Bishop KM., Ozaki HS. (2006) Recombinant inbreeding in mice reveals thresholds in embryonic corpus callosum development. Genes Brain Behav. (5): 170-188

Wang Y., Thekdi N., Smallwood P.M., Macke J.P., Nathans J. (2002) Frizzled-3 is required for the development of major fiber tracts in the rostral CNS. $J$ Neurosci. (22): 8563-8573

Weissman T., Noctor S.C., Clinton B.K., Honig L.S., Kriegstein A.R. (2003) Neurogenic radial glial cells in reptile, rodent and human: from mitosis to migration. Cereb Cortex. (13): 550-559

Williams M.E., Strickland P., Watanabe K., Hinck L. (2003) UNC5H1 induces apoptosis via its juxtamembrane region through an interaction with NRAGE. $J$ Biol Chem. (278): 17483-17490

Williams ME., Lu X., McKenna WL., Washington R., Boyette A., Strickland P., Dillon A., Kaprielian Z., Tessier-Lavigne M., Hinck L. (2006) UNC5A promotes neuronal apoptosis during spinal cord development independent of netrin-1. Nat Neurosci. (9): 996-998

Wilson D.A., Kadohisa M., Fletcher M.L. (2006) Cortical contributions to olfaction: plasticity and perception. Semin Cell Dev Biol. (17): 462-470

Wu W., Wong K., Chen J., Jiang Z., Dupuis S., Wu J.Y., Rao Y. (1999) Directional guidance of neuronal migration in the olfactory system by the protein Slit. Nature (400):331-336 
Zaki PA., Quinn JC., Price DJ. (2003) Mouse models of telencephalic development. Curr Opin Genet Dev. (13): 423-437 


\section{ACKNOWLEDGEMENTS}

First of all, I would like to thank Prof. Dr. Peter Gruss for giving me the opportunity of working in his group and for his scientific and moral support that was extremely important for my motivation during this study. I am deeply grateful to him for showing constant interest in my project and for giving advices that helped me in overcoming the difficulties appeared during the course of this study. It is extremely kind of him that he has been spending his time as the referee of my Ph.D thesis in spite of his really tight schedule as being the president of Max-Planck Society.

I would also like to thank PD Dr. Sigrid Hoyer-Fender for her being the coreferee of my thesis project and for spending her valuable time in evaluating my thesis. I am also grateful to all other members of my Ph.D thesis and examination committees for their involvement and valuable assistance.

I am very deeply grateful to Dr. Kamal Chowdhury for all his technical and theoretical supervision during my study. I had a continuous motivational support from him during the whole course of my work. His interest and kind help in every experimental result that I obtained finally made it possible to bring this study up to its current end. I would also like to thank PD Dr. Anastassia Stoykova for the initiation of the 'Cortex-Microarray' project and also for the valuable help with her impressive neuroanatomical knowledge.

I would like to thank Sabine Geisendorf and Andrea Conrad for their very helpful technical assistance and also for creating a friendly atmosphere in the lab during all these years. I would also like to thank Sigurd Hille for thousands of sequencings; Ulrich Franke for all microinjections and aggregations, Sharif Mahsur for all ES-cell electroporations. I am grateful to Monika Schindler and Simone Schmidt from the transgenic facility of MPI-Exp.Med. for the blastocyst injections.

I am thankful to Dr. Ulrike Teichmann, chief of our animal facility, for helpful discussions and interest in my work. I am extremely grateful to Alexandra Driehorst, Heike Fett, Alexandra Kurth, and Simone Brauer for taking care of all my mouse lines in the most professional level. 
I would like to thank PD Dr. Ahmed Mansouri, and Prof. Dr. Michael Kessel for their helpful scientific advices during my study; and very special thanks are due to Marianne Schuster and Anja Dietrich for helping me to deal with administrative problems.

Finally, I would like to express my greatest gratitude to my parents and my sister for their presence and continuous support in my life. My most special thanks are for Olga because of her being always near me and supporting me in every aspect of life. 


\title{
CURRICULUM VITAE
}

\author{
Persönliche Daten: Ahmet $\boldsymbol{U C A R}$ \\ JüdenStr.13a \\ 37073 Göttingen \\ geboren am 08 April 1977, in Konya / Türkei \\ türkischer Staatsbürger \\ 1983 - $1988 \quad$ Grundschule, Konya / Türkei \\ $1988-1995 \quad$ Gymnasium, Konya / Türkei \\ 1995 - 1999 Studium der Molekulare Biologie und Genetik (Diplom B.Sc), \\ Bilkent Universität, Ankara / Türkei \\ 1998 - $1999 \quad$ Diplomarbeit, Thema: \\ "Identification of the correlation between bladder cancer risk \\ and genetic polymorphisms in Turkish population" \\ AG Prof. Dr. Tayfun Özcelik, \\ Bilkent Universität, Ankara / Türkei \\ Publikationen: \\ - Toruner GA., Ucar A., Tez M., Cetinkaya M., Ozen H., Ozcelik T. \\ P53 codon 72 polymorphism in bladder cancer- no evidence of \\ association with increased risk or invasiveness. \\ Urol Res. 2001 Dec; 29(6): 393-5.
}

- Toruner GA., Akyerli C., Ucar A., Aki T., Atsu N., Ozen H., Tez M., Cetinkaya M., Ozcelik T.

Polymorphisms of glutathione S-transferase genes (GSTM1, GSTP1, and GSTT1) and bladder cancer susceptibility in the Turkish population

Arch Toxicol. 2001 Oct; 75(8): 459-64

1999 - $2001 \quad$ Studium der Molekulare Biologie und Genetik (Diplom M.Sc), Bilkent Universität, Ankara / Türkei

1999 - $2001 \quad$ Diplomarbeit, Thema:

"The expression of BCL-2 family of genes during liver regeneration"

AG: PD Dr. Kamil C. Akcali,

Bilkent Universität, Ankara / Türkei

Publikationen:

- Akcali KC., Dalgic A., Ucar A., Haj KB., Guvenc D.

Expression of bcl-2 gene family during resection induced liver regeneration: comparison between hepatectomized and sham groups.

World J Gastroenterol. 2004 Jan 15; 10(2): 279-83 
2001 (Oct)-2007 Promotion, Thema:

"Identification and functional characterization of $u n c 5 \mathrm{~A}$ during neocortical regionalization"

Abteilung: Prof. Dr. Peter Gruss,

Max-Planck-Institut f. biophysikalische Chemie, Göttingen

Publikationen:

- Yuh-Shin Chang*, Kamal Chowdhury*, Joachim Glaubitz*, Friederike Kirsch*, Victoria Moreno*, Sven Muhlfriedel*, Kay Nieselt-Struwe*, Anasstassia Stoykova*, Ahmet Ucar* and Peter Gruss (* names in alphabetical order, contributed equally)

Large-scale microarray screen for genes with regionalized expression in the developing mouse cerebral cortex.

MPINews, April 2002

- Fimia GM., Stoykova A., Romagnoli A., Giunta L., Di Barolomeo S., Ucar A., Nardacci R., Corazzari M., Schwartz P., Gruss P., Piacentini M., Chowdury K., and Cecconi F.

The novel autophagy regulator Ambra-1 is required for nervous system development

Submitted to Nature, $3^{\text {rd }}$ and final review in progress.

- Ucar A., et.al.

Identification of new marker genes for E14.5 cerebral cortex by DNA Microarray screening and verification with in situ hybridization.

Manuscript in preparation

- Ucar A., et.al.

Ectopic overexpression of unc $5 A$ in developing distal neuroretina causes background-dependent blindness

Manuscript in preparation

- Ucar A., et.al.

unc5A deficiency causes background-dependent blindness in 129XCD1 mixed background.

Manuscript in preparation 